\title{
8 Ergebnisse
}

Nachfolgend werden die Ergebnisse der vier einzelnen Untersuchungen zunächst wertfrei dargelegt, d. h. die Inhalte dieses Kapitels entsprechen den Sichtweisen der Behörden bzw. der Journalisten. Die Bewertung der Ergebnisse erfolgt in Kapitel 9. Zuerst werden die Ergebnisse zur behördlichen Risiko- und Krisenkommunikation (Kap. 8.1, 8.2) ausgeführt, anschließend die zur medialen Logik im Kontext von Risiken und Krisen (Kap. 8.3, 8.4). Die Ergebnisse werden in Anlehnung an die Forschungsfragen der Arbeit und die sich daraus ableitenden Kategoriensysteme dargestellt.

\subsection{Risiko- und Krisenkommunikation von Behörden: Ergebnisse der Dokumentenanalyse}

Der erste Gliederungspunkt beschreibt das Untersuchungsmaterial anhand formaler Kriterien. Alle darauffolgenden Gliederungspunkte beziehen sich auf den Inhalt der Dokumente.

\subsubsection{Darstellung des Untersuchungsmaterials}

Die Dokumente der systematischen Recherche sind Publikationen von folgenden Organisationen: AKNZ, BBK, BfR, BLE, BMBF, BMEL, BMI, BMG, BVL, Deutscher Städtetag, RKI sowie dem Innenministerium und dem Landesgesundheitsamt von Baden-Württemberg. Folgende Dokumenttypen liegen am häufigsten vor: Berichte, Handbücher, Leitfäden, Broschüren und Forschungsberichte.

Die Dokumente der systematischen Auswertung wurden im Zeitraum von August 2003 bis zum Februar 2013 veröffentlicht. Der Umfang variiert von einer Seite bis zu 766 Seiten.

Die Dokumente sollen folgenden Zielen dienen: Informationsvermittlung, konzeptionelle Hilfen, praktische Empfehlungen, Überblick des Übungs- und Weiterbildungsangebotes. Als Zielgruppen der Dokumente lassen sich Behörden, Unternehmen und Fachkreise identifizieren. Behördliche Kommunikationsver- 
antwortliche werden in zwei Dokumenten explizit adressiert (Bundesministerium des Innern 2008 ${ }^{29}$; Deutscher Städtetag 2012).

Die Ergebnisse der Analyse zeigen eine anwendungsorientierte, praxisnahe Ausrichtung der gesichteten Dokumente.

\subsubsection{Risikokommunikation}

\subsubsection{Verständnisse von Risikokommunikation}

In zehn der 23 B-Dokumente liegen folgende Verständnisse und Deutungsmuster von Risikokommunikation vor:

\section{Dialog:}

In sieben Dokumenten wird Risikokommunikation als ein interaktiver, zielgerichteter Austausch von Informationen und Meinungen über Risiken verstanden. Ein Dialog mit Bürgern sollte erreicht werden (Bundesministerium des Innern 2008; 2009; Bundesamt für Bevölkerungsschutz und Katastrophenhilfe \& Deutscher Städtetag 2010; Bundesamt für Bevölkerungsschutz und Katastrophenhilfe 2011a; 2011d; Weber \& Dieker 2012; Böl 2013).

\section{Staatlicher Auftrag:}

Risikokommunikation wird als ein wesentlicher Bestandteil staatlichen Handelns (Schröder 2011), sogar als eine unverzichtbare, staatliche Verpflichtung gesehen (Bundesamt für Bevölkerungsschutz und Katastrophenhilfe \& Deutscher Städtetag 2010: 54).

\section{Partizipatives Verständnis:}

Risikokommunikation wird als Voraussetzung für eine aktive Teilnahme der Bürger am interaktiven Austausch über Risiken und der Teilhabe an politischen Entscheidungsprozessen über Risiken verstanden (Bundesministerium des Innern 2008; Weber \& Dieker 2012).

\section{Teil des Risikomanagements:}

Risikokommunikation umfasst Kommunikation über die Identifizierung, Analyse, Bewertung sowie das Management von Risiken bzw. baut darauf auf (Bun-

\footnotetext{
${ }^{29}$ Wie in Kapitel 7.1.2.1 dargelegt wird nachfolgend ebenfalls die überarbeitete Auflage des Leitfadens „Krisenkommunikation“ des BMI aus dem Jahr 2014 berücksichtigt. Zentrale Neuerungen in der überarbeiteten Auflage betreffen separate Kapitel bzw. Teilkapitel über Risikokommunikation, Social Media und Krisenmanagement. Insgesamt ist das Dokument kürzer und visuell anschaulicher aufbereitet. Einzelne Neuerungen werden in den entsprechenden Abschnitten ausgeführt.
} 
desamt für Bevölkerungsschutz und Katastrophenhilfe \& Deutscher Städtetag 2010; Bundesamt für Bevölkerungsschutz und Katastrophenhilfe 2011d; Weber \& Dieker 2012).

Kombinierte Verständnisse:

In drei Dokumenten existieren Verständnisse, die verschiedene der hier aufgeführten Deutungen kombinieren (Böl 2013; Bundesministerium für Ernährung, Landwirtschaft und Verbraucherschutz 2013). In einem dieser wird zwischen interner und externer Risikokommunikation differenziert. Interne Risikokommunikation wird demnach als Teil des Risikomanagements betrachtet. Externe Risikokommunikation wird als ein adressatengerechter Dialog, etwa mit Medien, verstanden (Bundesamt für Bevölkerungsschutz und Katastrophenhilfe 2011d).

\subsubsection{Ziele und Zielgruppen von Risikokommunikation}

Die folgenden Aufgaben und Ziele ${ }^{30}$ von Risikokommunikation konnten mit entsprechender Häufigkeit in den Dokumenten identifiziert werden:

- $\quad$ Sensibilisieren, aufklären und informieren (4x)

- Verhaltensänderungen auslösen (3x)

- $\quad$ Selbstständiger Umgang mit Risiken in der Bevölkerung ermöglichen $(2 \mathrm{x})$

- Transparente Kommunikation (1x)

- Herausbildung einer Risikokultur (1x)

- Schaffung von Glaubwürdigkeit und Vertrauen (1x)

- $\quad$ Auf Krisen vorbereiten (1x)

Folgende Zielgruppen von Risikokommunikation werden in den Dokumenten genannt:

- $\quad$ Bürger (6x)

- $\quad$ Staatliche Organisationen (Bundes-, Landes-, Kommunalebene) (4x)

- $\quad$ Medien (3x)

- Unternehmen (2x)

- $\quad$ Fachöffentlichkeiten $(2 x)$

- Interessensvertreter $(2 \mathrm{x})$

- $\quad$ Mitarbeiter $(1 \mathrm{x})$

\footnotetext{
${ }^{30}$ Innerhalb der Dokumente wird nicht immer eindeutig zwischen Aufgaben und Zielen differenziert, so dass beide Begriffe in der Ergebnisdarstellung synonym verwendet werden. Dies gilt auch für die Krisenkommunikation sowie für die Ergebnisdarstellung der Experteninterviews.
} 


\subsubsection{Voraussetzungen von Risikokommunikation}

In den Dokumenten werden verschiedene Voraussetzungen dargelegt, die für eine gelungene Risikokommunikation berücksichtigt werden sollten.

\section{Interne Risikokommunikation:}

Eine gelungene interne Risikokommunikation gilt in einem Dokument als Voraussetzung für eine adäquate externe Risikokommunikation (Bundesamt für Bevölkerungsschutz und Katastrophenhilfe 2011d).

Berücksichtigung aller Akteure/Wissenschaftlicher Dissens:

Es ist wichtig, alle betroffenen Akteure frühzeitig am Risikokommunikationsprozess zu beteiligen (Bundesministerium des Innern 2008; Bundesamt für Bevölkerungsschutz und Katastrophenhilfe 2011d; Weber \& Dieker 2012). Dabei sollten divergierende, wissenschaftliche Meinungen berücksichtigt und kommuniziert werden, um einen Expertenstreit in der Krise zu vermeiden (Robert Koch-Institut 2007; Bundesamt für Bevölkerungsschutz und Katastrophenhilfe 2011d). Das bedeutet, dass es nicht nur eine zu kommunizierende Meinung gibt, sondern auch widerstreitende Positionen und Unsicherheiten transparent vermittelt werden sollten (Böl 2013).

\section{Kontinuität:}

Risikokommunikation sollte als Daueraufgabe verstanden werden, die unabhängig von einem konkreten Anlass geführt wird (Bundesministerium des Innern 2008; Weber \& Dieker 2012).

\section{Koordination/Zusammenarbeit:}

Die Koordination verschiedener interner und externer Kommunikationsprozesse, inklusive der Verständigung über Zuständigkeiten, sowie nationale und internationale Zusammenarbeit sind für Risikokommunikation erforderlich (Weber \& Dieker 2012; Böl 2013). Insbesondere bei einer Pandemie sollten die zahlreichen Akteure des Gesundheitssystems auf allen Ebenen eine konsistente Kommunikation gewährleisten (Robert Koch-Institut 2007; 2015).

\section{Forschungstätigkeiten:}

Sozialwissenschaftliche Forschung und Analysen zu den Themen Risikowahrnehmung, Umgang mit Risiken und Informationsverhalten der Bevölkerung sind Voraussetzungen für eine gelungene Risikokommunikation (Weber \& Dieker 2012; Böl 2013).

\section{Empfehlungen:}

Offenheit, Transparenz, Verlässlichkeit, Eindeutigkeit, Zuverlässigkeit, Glaub- 
würdigkeit, Festlegung von Kommunikationszielen sowie schnelle, frühzeitige und regelmäßige Informationen werden für eine gelungene Risikokommunikation empfohlen (Bundesamt für Bevölkerungsschutz und Katastrophenhilfe \& Deutscher Städtetag 2010; Bundesamt für Bevölkerungsschutz und Katastrophenhilfe 2011d; Weber \& Dieker 2012; Böl 2013).

\subsubsection{Instrumente von Risikokommunikation}

Insbesondere für die Behörden BfR, BBK und BMI sind Risikokommunikationsinstrumente erkennbar. Darunter zählen klassische Instrumente der Presse- und Öffentlichkeitsarbeit. Das BfR veranstaltet zudem runde Tische, Expertengespräche, wissenschaftliche Kongresse, Schülerworkshops, Science Slams und Sommerschulen (Böl 2013).

Außerdem lassen sich folgende Instrumente identifizieren: der Leitfaden „Schutz Kritischer Infrastrukturen“ (Bundesministerium des Innern 2009), die Website des BBK, das Internetangebot des BBK für Kinder und Jugendliche „Max und Flocke“, Flyer, Broschüren sowie die Teilnahme an Fachmessen (Weber \& Dieker 2012; Weber 2013). Es wird ausgeführt, dass Krisenübungen wie die LÜKEX mit entsprechender Medienarbeit für die Risikokommunikation genutzt werden können (Bundesamt für Bevölkerungsschutz und Katastrophenhilfe 2011b).

Als Risikokommunikationsinstrumente im Bereich der ENV werden die Website www.ernaehrungsvorsorge.de sowie Broschüren betrachtet (Gizewski 2012).

Die zunehmende Bedeutung von Social Media (Facebook, Twitter, Blogs) wird in fünf Dokumenten verdeutlicht (Bundesamt für Bevölkerungsschutz und Katastrophenhilfe 2011b; Schröder 2011; Weber \& Dieker 2012; Deutscher Städtetag 2012; Bundesministerium des Innern 2014). Das BBK stellt dabei heraus, dass Social Media eine Chance für die Optimierung der Risikokommunikation mit der Bevölkerung darstellen, insbesondere, da sie Dialog und Diskussion ermöglichen (Weber \& Dieker 2012). Es wird aufgeführt, dass im BBK an einem Social-Media-Konzept und an Apps gearbeitet wird (Schröder 2011; Weber \& Dieker 2012).

In Bezug auf Social Media stellt der überarbeitete Leitfaden des BMI (2014) ein Novum dar. Er enthält ein eigenes Teilkapitel dazu, das ausführt, was Social Media sind, wie sie Kommunikationsprozesse verändern und dass die Nutzung von Social-Media-Kanälen einen Mehrwert für die Deutungshoheit der Behörden bieten kann. Dabei wird betont, dass Social Media als Teil einer gesamten Risiko- und Krisenkommunikationsstrategie verstanden und nicht nur in der aku- 
ten Krise genutzt werden sollten. Entsprechend werden umfassende Ratschläge zur Entwicklung einer Social-Media-Strategie geliefert, zusätzlich gibt es im Anhang des Dokuments Social-Media-Guidelines (Bundesministerium des Innern 2014).

\subsubsection{Krisenkommunikation}

\subsubsection{Verständnisse von Krisenkommunikation}

In neun der 23 B-Dokumente sind Verständnisse von Krisenkommunikation erkennbar.

\section{Teil des Krisenmanagements:}

Krisenkommunikation wird in vier Dokumenten als wichtiger Teil des (staatlichen) Krisenmanagements betrachtet (Bundesamt für Verbraucherschutz und Lebensmittelsicherheit 2006; Robert Koch-Institut 2007; Bundesministerium des Innern 2008; Deutscher Städtetag 2012). Dass Krisenkommunikation eine zentrale Bedeutung für eine erfolgreiche Krisenbewältigung hat, wird drei Mal erwähnt (Bundesamt für Bevölkerungsschutz und Katastrophenhilfe 2005; 2011d; Bundesministerium des Innern 2008).

\section{Dialog:}

Analog zur Risikokommunikation lässt sich bei der Krisenkommunikation in sechs Dokumenten ein dialogisches Verständnis identifizieren. Demnach ist Krisenkommunikation ein Austausch von Informationen und Meinungen während einer Krise. Damit gehen Ratschläge, dass keine einseitige Kommunikation stattfinden sollte und den Betroffenen Rückkanäle eröffnet werden sollten einher (Bundesamt für Bevölkerungsschutz und Katastrophenhilfe 2011a; 2011d; Bundesamt für Bevölkerungsschutz und Katastrophenhilfe \& Deutscher Städtetag 2010; Bundesamt für Verbraucherschutz und Lebensmittelsicherheit 2006; Bundesministerium des Innern 2008; Robert Koch-Institut 2007).

\section{Allgemeines Verständnis:}

Zusätzlich liegt ein allgemeines Verständnis vor, das Krisenkommunikation als alle ,kommunikativen Aktivitäten, die in Zusammenhang mit einer Krise durchgeführt werden " (Bundesministerium des Innern 2005: 52), betrachtet.

\subsubsection{Ziele und Zielgruppen von Krisenkommunikation}

Die nachfolgenden Ziele von Krisenkommunikation lassen sich in den Dokumenten identifizieren: 
- $\quad$ Festigung von Vertrauen und Glaubwürdigkeit ${ }^{31}(5 x)$

- $\quad$ Über Gründe und Folgen einer Krise informieren(3x)

- Kommunikationsflüsse zwischen allen Beteiligten gewährleisten (3x)

- $\quad$ Reputation der Behörden wahren (2x)

- Medienberichterstattung sicherstellen (1x)

- Stabilität nach innen und außen herstellen (1x)

Als Zielgruppen von Krisenkommunikation können die gleichen wie bei der Risikokommunikation festgestellt werden (vgl. Kap. 8.1.2.2).

\subsubsection{Voraussetzungen von Krisenkommunikation}

Die nachfolgenden Voraussetzungen werden für eine möglichst gelungene Krisenkommunikation aufgeführt.

\section{Vorbereitung und Übungen:}

Zwei Dokumente verweisen auf eine gründliche Vorbereitung und regelmäßige Übungen mit allen Akteuren als wichtige Voraussetzungen für Krisenkommunikation (Bundesamt für Bevölkerungsschutz und Katastrophenhilfe \& Deutscher Städtetag 2010; Weber 2013).

\section{Krisenkommunikationsstrategien:}

In fünf Dokumenten wird geäußert, dass Krisenkommunikation bereits zuvor entwickelte Kommunikationsstrategien benötigt (Bundesministerium des Innern 2005; 2008; Robert Koch-Institut 2007; Bundesamt für Bevölkerungsschutz und Katastrophenhilfe \& Deutscher Städtetag 2010; Bundesamt für Bevölkerungsschutz und Katastrophenhilfe \& Landesgesundheitsamt Baden-Württemberg 2010). Diese sollten sich bestmöglich an bestimmten Szenarien orientieren (Bundesministerium des Innern 2008). Ein Maßnahmenkatalog mit konkreten Anweisungen für Mitarbeiter wird empfohlen, um Arbeitsroutine in der Krise ermöglichen zu können (ebd.). Gleichermaßen wird darauf verwiesen, dass jede Krise individuell ist und Konzepte und Strategien daher kurzfristig angepasst und aktualisiert werden müssen (ebd.).

\section{Journalistisches Netzwerk:}

Eine gute Beziehungspflege zu den wichtigsten Journalisten gilt ebenfalls als Voraussetzung für eine gelungene Krisenkommunikation (Weber 2013).

\footnotetext{
${ }^{31}$ Glaubwürdigkeit und Vertrauen werden in vielen Dokumenten als wichtige Faktoren sowohl für Risiko- als auch für Krisenkommunikation genannt. Dabei werden sie unterschiedlich als Ziel, Voraussetzung, Herausforderung oder auch als Strategie betrachtet.
} 


\subsubsection{Instrumente von Krisenkommunikation}

Instrumente, die für eine Krise empfohlen werden, sind: Darksites (4x), Pressemitteilungen (3x), Hintergrundinformationen (3x), Websites (3x), Hotlines (3x), Pressekonferenzen (2x), FAQs (2x), Medienbeobachtung und -auswertung (1x), Interviews (1x), interne Besprechungen (1x). Es wird mehrfach darauf hingewiesen, dass die Instrumente bestmöglich schon vorbereitet sind, z. B. Mustertexte für Pressemitteilungen, Sprechzettel (z. B. Bundesministerium des Innern 2008; Bundesamt für Bevölkerungsschutz und Katastrophenhilfe 2011d).

Die genannten Aspekte zu Social Media für die Risikokommunikation gelten größtenteils auch für die Krisenkommunikation, insbesondere die Ausführungen des überarbeiteten BMI-Leitfadens (2014). Im Leitfaden des Deutschen Städtetags (2012) fällt auf, dass Social Media in der Einleitung mehrfach als bedeutend für Krisenkommunikation herausgestellt werden. Bei den folgenden Handlungsempfehlungen gibt es jedoch keinen erneuten Bezug dazu, was eine Divergenz zum vorherigen Fokus darstellt (ebd.).

\subsubsection{Praktische Hilfen und Empfehlungen für Krisenkommunikation}

In sechs B-Dokumenten und zwei E-Dokumenten gibt es Planungshilfen und Checklisten, die die Umsetzbarkeit von Risiko- und Krisenkommunikation in der Praxis unterstützen und erleichtern sollen (Innenministerium Baden-Württemberg 2003; Bundesministerium des Innern 2005; 2008; Robert Koch-Institut 2007; Bundesamt für Bevölkerungsschutz und Katastrophenhilfe \& Innenministerium Baden-Württemberg 2010; Bundesamt für Bevölkerungsschutz und Katastrophenhilfe \& Landesgesundheitsamt Baden-Württemberg 2010; Bundesamt für Bevölkerungsschutz und Katastrophenhilfe 2011d; Deutscher Städtetag 2012). Diese sind thematisch breit gefächert und umfassen u. a. auch technische Voraussetzungen für die Krisenkommunikation. Zusätzlich gibt es beigefügte Fragenkataloge, Hinweise oder ein Glossar für einheitliche Verständnisse (Bundesministerium des Innern 2005; 2008).

Aufgrund der praktischen Ausrichtung der Dokumente lassen sich zahlreiche Empfehlungen, wie Behörden in Krisen kommunizieren sollten, identifizieren. Nachfolgend werden zentrale Empfehlungen aus sechs einschlägigen Dokumenten mit besonders vielen Empfehlungen exemplarisch aufgezeigt. Diese sind in strategische und operative Empfehlungen systematisiert (Robert Koch-Institut 2007; Bundesministerium des Innern 2008; Bundesamt für Bevölkerungsschutz und Katastrophenhilfe \& Deutscher Städtetag 2010; Bundesamt für Bevölkerungsschutz und Katastrophenhilfe 2011d; Deutscher Städtetag 2012; Weber 2013). 
- Offensiv kommunizieren/Initiative ergreifen (5x)

- Transparent und ehrlich kommunizieren $(5 \mathrm{x})$

- One-voice-policy befolgen (4x)

- Zielgruppen verstehen und Kommunikationsbotschaften den Bedürfnissen der Zielgruppen anpassen (3x)

- $\quad$ Person auf höchster Leitungsebene sprechen lassen (3x)

- Glaubwürdig und konsistent kommunizieren (2x)

- $\quad$ Anforderungen der Medien berücksichtigen (1x)

- Wissen und Nicht-Wissen kommunizieren (1x)

\section{Operative Empfehlungen}

- $\quad$ Schnell informieren $(5 x)$

- Kontinuierlich informieren $(5 x)$

- Nicht spekulieren, vermuten $(4 \mathrm{x})$

- Nicht „Kein Kommentar“ sagen (3x)

- $\quad$ Sachlich bleiben $(2 \mathrm{x})$

- Sicher, kontrolliert und überlegt sprechen $(2 x)$

- $\quad$ Nicht lügen, nicht ausweichen (2x)

- $\quad$ Alltägliche Sprache verwenden $(2 x)$

- Wichtige Dinge wiederholen $(2 \mathrm{x})$

- Wissenslücken zugeben $(2 \mathrm{x})$

- $\quad$ Klare Botschaften vermitteln $(2 \mathrm{x})$

- Rückkopplungsmöglichkeit für dialogische Kommunikation anbieten $(2 \mathrm{x})$

- Medien bestmögliche Berichterstattung vor Ort gewähren (1x)

- Viele und anschauliche Beispiele verwenden (1x)

\subsubsection{Herausforderungen der Krisenkommunikation}

In einem Teil der Dokumente werden die nachfolgenden fünf zentralen Herausforderungen thematisiert.

Öffentliche Aufmerksamkeit, dadurch Mangel an personellen Ressourcen: Im Falle einer Krise ist mit einem erhöhten, teils sehr starkem Interesse seitens der Medien und Bevölkerung zu rechnen (Robert Koch-Institut 2007; Bundesministerium des Innern 2008). Dies ist mit einer Vielzahl von Anfragen verbunden (Bundesamt für Verbraucherschutz und Lebensmittelsicherheit 2006; Bundesamt für Bevölkerungsschutz und Katastrophenhilfe \& Deutscher Städtetag 2010). Für 
die Krisenkommunikation der verantwortlichen Behörden bedeutet das eine besondere Herausforderung für die personellen Ressourcen (Bundesamt für Verbraucherschutz und Lebensmittelsicherheit 2006; Robert Koch-Institut 2007). Aus diesem Grund sollten insbesondere bei einer sich langsam entwickelnden Krise wie einer Pandemie bereits im Vorfeld die schnelle Verfügbarkeit und Vermittlung von Informationen gewährleistet werden (Robert Koch-Institut 2007; 2015). Außerdem sollte dafür Sorge getragen werden, dass in der akuten Krise Personal zur Verstärkung angefordert werden kann (Bundesamt für Bevölkerungsschutz und Katastrophenhilfe 2011d).

\section{Plötzlichkeit:}

Das plötzliche Eintreten einer Krise ist eine der größten Herausforderungen der Krisenkommunikation, da kommuniziert werden muss, ohne unter Umständen vollständige Informationen vorliegen zu haben. Zeit für Überprüfungen und Abwägungen ist meist nur bedingt vorhanden (Bundesministerium des Innern 2008; Bundesamt für Bevölkerungsschutz und Katastrophenhilfe \& Deutscher Städtetag 2010). Dadurch können widersprüchliche und zur Verunsicherung führende Aussagen der behördlichen Akteure resultieren (Schröder 2011). Die akute Krise erfordert schnelles Handeln und Kommunizieren, weshalb Abstimmungsprozesse beschleunigt stattfinden müssen. Hierfür sollten bereits im Vorfeld rasche Entscheidungs- und Kommunikationswege vorbereitet sein (Bundesministerium des Innern 2005; Robert Koch-Institut 2007).

\section{Schnelligkeit der Kommunikation/Social Media:}

Medien verarbeiten Informationen zunehmend schneller und verbreiten sie direkter. Behörden sollten verstehen, dass sie die Krisenkommunikation nicht in Gänze steuern können (Deutscher Städtetag 2012). Diese Schnelligkeit der heutigen Medienwelt und die Kommunikation über Social Media können die Informationsqualität von Behörden erschweren, weil Informationen häufiger unvollständig und nicht wahrheitsgetreu vermittelt werden (Schröder 2011).

\section{Rechtliche Herausforderungen:}

Die Veröffentlichung sensibler Informationen kann in Krisen problematisch sein. Behörden sollten sorgfältig abwägen, welche Informationen aus rechtlicher Perspektive veröffentlicht werden dürfen und welche der Geheimhaltung unterliegen (Bundesamt für Bevölkerungsschutz und Katastrophenhilfe 2011d). In einem E-Dokument wird die Informationspflicht von Behörden angesprochen (Innenministerium Baden-Württemberg 2003). Behörden sind gegenüber Journalisten zur Auskunft verpflichtet, was zu einer Herausforderung für die Behörde werden kann. Nur in bestimmten rechtlichen Fällen kann die Informationspflicht außer Kraft gesetzt werden (ebd.). 


\subsubsection{Zusammenhängende Betrachtung}

In sechs B-Dokumenten kann eine zusammenhängende Betrachtung von Risikound Krisenkommunikation identifiziert werden. In anderen Dokumenten, in denen Risikokommunikation und Krisenkommunikation erwähnt werden, werden sie synonym, ohne Abgrenzung zueinander genannt (Bundesministerium des Innern 2005; Bundesamt für Bevölkerungsschutz und Katastrophenhilfe \& Deutscher Städtetag 2010). In einem Dokument wird die undifferenzierte Verwendung der Begriffe Risiko- und Krisenkommunikation, auch in Fachkreisen, kritisiert (Weber \& Dieker 2012). Darüber hinaus gibt es Dokumente, in denen jeweils nur eine Disziplin thematisiert wird (z. B. Deutscher Städtetag 2012; Bundesministerium für Ernährung, Landwirtschaft und Verbraucherschutz 2013).

\section{Risikokommunikation als Grundlage von Krisenkommunikation}

In sechs B-Dokumenten wird konstatiert, dass eine langfristig umgesetzte Risikokommunikation als Voraussetzung für eine gelungene Krisenkommunikation gilt (Bundesministerium des Innern 2008; Bundesamt für Bevölkerungsschutz und Katastrophenhilfe \& Innenministerium Baden-Württemberg 2010; Schröder 2011; Weber \& Dieker 2012; Weber 2013).

\section{Gemeinsamkeiten und Unterschiede von Risiko- und Krisenkommunikation}

In zwei B-Dokumenten und einem E-Dokument werden Gemeinsamkeiten und Unterschiede beider Disziplinen skizziert (Dickmann et al. 2007; Weber \& Dieker 2012; Bundesministerium des Innern 2014).

Beiden Disziplinen liegen dieselben Prinzipien der Kommunikation zugrunde: Offenheit, Transparenz, Glaubwürdigkeit, Konsistenz, Ehrlichkeit und Dialogorientierung (Weber \& Dieker 2012; Bundesministerium des Innern 2014).

Die zeitliche Dimension wird als wesentlicher Unterschied von Risiko- und Krisenkommunikation gekennzeichnet. Während Risikokommunikation präventiv auf Risiken vorbereiten und ein dauerhaftes Vertrauensverhältnis zu den Zielgruppen herstellen soll, kennzeichnet sich Krisenkommunikation durch kurzfristiges, zeitlich begrenztes Handeln. Krisenkommunikation ist demnach anlassbezogen, Risikokommunikation sollte hingegen als permanente Kommunikation praktiziert werden (Dickmann et al. 2007; Weber \& Dieker 2012; Bundesministerium des Innern 2014). 
Darüber hinaus wird darauf verwiesen, dass bei bestimmten Krisen, die lange andauern, wie eine Pandemie, nur schwer zwischen Risiko- und Krisenkommunikation differenziert werden kann (Weber \& Dieker 2012). Solche lang andauernden Situationen stellen eine besondere Herausforderung für die Kommunikation dar: Mit der Risikokommunikation versucht man langfristig Verhaltensweisen zu beeinflussen, in der kurzfristigen Krisensituation muss dann unter Entscheidungszwang und Ungewissheit kommuniziert werden (Dickmann et al. 2007).

\section{Phasenverständnisse}

Dass eine Krise in verschiedenen Phasen abläuft, berücksichtigen vier Dokumente. Zum einen wird der Verlauf dreiphasig in die Frühbeobachtung, das eigentliche Krisenereignis und die Krisenbewältigung eingeteilt (Bundesamt für Verbraucherschutz und Lebensmittelsicherheit 2006). Zum anderen wird vierphasig zwischen potenzieller, latenter und akuter Krisenphase sowie der Nachkrisenphase differenziert (Bundesministerium des Innern 2008). In der neuen Auflage des Leitfadens des Bundesministeriums des Innern (2014: 6f, 35) wird das Krisenmanagement als ein Kreislauf bestehend aus den vier Phasen Vorsorge, Vorbereitung, Bewältigung und Nachbereitung verstanden. Obgleich sich dieses Phasenverständnis nicht explizit auf Kommunikationsprozesse bezieht, kann es als ein vergleichsweise komplexes integratives Verständnis aufgefasst werden, auch weil die Anmerkung erfolgt, dass Krisen nicht linear verlaufen (ebd.: 6). Eine Neuerung des Leitfadens stellt eine „Arbeitshilfe“ zur Nachbereitung der Krisenkommunikation dar, die im Anhang eine Checkliste dafür zur Verfügung stellt. Organisationales Lernen wird am Rande erwähnt (ebd.: 49f).

Für die Kommunikationsverantwortlichen in einer Pandemie ist das phasenorientierte Vorgehen von großer Bedeutung, da sich der Nationale Pandemieplan mit seinen Empfehlungen für die Kommunikation an den Phasen einer Pandemie orientiert, diese sind in Anlehnung an die WHO-Pandemiephasen gehalten (Robert Koch-Institut 2007: 6 Phasen; Robert Koch-Institut 2015: 4 Phasen). Entsprechend sind die derzeitigen Empfehlungen zur Risiko- und Krisenkommunikation während eines pandemischen Geschehens nach den vier Phasen „Interpandemischer Zeitraum“, „Erste Einzelfälle und Beginn der Übertragung in der Bevölkerung“, „Anhaltende Übertragung in der Bevölkerung“ und „Übergang in einen interpandemischen Zeitraum" ausgerichtet (ebd.: 4ff). 
Dass Krisenkommunikation im Nachgang einer Krise zu evaluieren ist, wird in vier Dokumenten erwähnt (Bundesamt für Verbraucherschutz und Lebensmittelsicherheit 2006; Bundesministerium des Innern 2008; Bundesamt für Bevölkerungsschutz und Katastrophenhilfe 2011d; Deutscher Städtetag 2012). Mit einer Evaluation sollte geklärt werden, was man aus der Krise lernen konnte, was getan werden kann, um die möglichen Krisenursachen langfristig zu beseitigen und wann ein erhöhtes Medieninteresse wieder auftreten könnte, etwa weil sich das Ereignis jährt (Bundesministerium des Innern 2008; Deutscher Städtetag 2012). Die Evaluation der Risikokommunikation wird in zwei Dokumenten erwähnt (Bundesamt für Bevölkerungsschutz und Katastrophenhilfe 2011d; Robert Koch-Institut 2015).

\subsubsection{Krisenübungen}

Es wird ausgeführt, dass Behörden bereits vor dem möglichen Eintritt einer Krise Verfahren des Krisenmanagements und der damit einhergehenden Risikound Krisenkommunikation trainieren und regelmäßig überprüfen sollten (Bundesamt für Bevölkerungsschutz und Katastrophenhilfe \& Deutscher Städtetag 2010; Bundesamt für Bevölkerungsschutz und Katastrophenhilfe 2011d; Deutscher Städtetag 2012). Zwei Dokumente empfehlen recht allgemein Medientrainings für Nicht-Medienerfahrene sowie die Aus- und Fortbildung von Mitarbeitern, etwa für Bürgertelefone (Bundesministerium des Innern 2008; Deutscher Städtetag 2012).

Zum BBK gehört die AKNZ, die Weiterbildungen in den Themenfeldern der Krisenprävention, -vorsorge und Gefahrenabwehr anbietet. Die Programme der AKNZ verdeutlichten einen hohen Stellenwert von praxisnahen Übungen und Fortbildungen. Es werden mehrere Seminare zu den Themen Risiko- und Krisenkommunikation für Mitarbeiter der Presse- und Öffentlichkeitsarbeit angeboten (Bundesamt für Bevölkerungsschutz und Katastrophenhilfe 2012b:215ff; 2013: 217ff).

\section{LÜKEX-Übungen}

Ein hoher Stellenwert der LÜKEX wird deutlich. Einige der analysierten Dokumente beschäftigen sich ausschließlich mit der Krisenmanagement-Übung und wurden aus Erfahrungen und Erkenntnissen vergangener LÜKEX-Übungen erstellt (Bundesamt für Bevölkerungsschutz und Katastrophenhilfe 2011b; 2011c; Bundesministerium des Innern \& Bundesministerium für Gesundheit 2007). 
Ein Hauptziel der LÜKEX ist es, die Zusammenarbeit von Bund und Bundesländern, von polizeilicher und nicht-polizeilicher Gefahrenabwehr sowie von öffentlichen und privaten Sektoren zu trainieren. Bestehende Strukturen sollen verbessert werden (Bundesministerium des Innern \& Bundesministerium für Gesundheit 2007; Bundesamt für Bevölkerungsschutz und Katastrophenhilfe 2011b). Das Üben und Verbessern einer abgestimmten Medien- und Öffentlichkeitsarbeit wird als ein weiteres Ziel der LÜKEX betrachtet (Bundesamt für Bevölkerungsschutz und Katastrophenhilfe 2011b; 2011c). Unterschieden wird zwischen der fiktiven, während der Übung stattfindenden Presse- und Öffentlichkeitsarbeit und der realen Presse- und Öffentlichkeitsarbeit mit dem Ziel, eine breite Medienberichterstattung über die LÜKEX zu generieren. Bisher gab es im Vorfeld der LÜKEX-Übungen Workshops für Pressesprecher (Bundesamt für Bevölkerungsschutz und Katastrophenhilfe 2011b).

Für die fiktive Medienarbeit wird in den Übungen eine virtuelle Medienlandschaft aus Fernseh-, Zeitungs- und Rundfunkbeiträgen konstruiert (Bundesamt für Bevölkerungsschutz und Katastrophenhilfe 2011c). Seit 2011 werden auch Social Media in die LÜKEX einbezogen, indem Facebook und Twitter simuliert werden (Bundesamt für Bevölkerungsschutz und Katastrophenhilfe 2012a; 2014c).

Es wird konstatiert, dass ein großes öffentliches und mediales Interesse an Stabsrahmenübungen wie der LÜKEX existiert, weshalb der realen Presse- und Öffentlichkeitsarbeit über die Übung eine hohe Bedeutung zugeschrieben wird (Bundesamt für Bevölkerungsschutz und Katastrophenhilfe 2011b). Die Medienarbeit sollte demnach als ein Instrument der Risikokommunikation genutzt werden, da die LÜKEX einen Anlass bietet, über die Zusammenarbeit der Akteure im Bevölkerungs- und Katastrophenschutz zu berichten. Idealerweise soll so das Vertrauen in die behördliche Krisenvorsorge ausgebaut werden. Die reale Medienarbeit sollte vor, während und nach der Übung erfolgen (Bundesamt für Bevölkerungsschutz und Katastrophenhilfe 2011b).

Die LÜKEX 2007 nahm eine Influenzapandemie mit mittelschwerem Ausmaß als Szenario für die Übung an (Bundesministerium des Innern \& Bundesministerium für Gesundheit 2007). Dabei spielte die fiktive Presse- und Öffentlichkeitsarbeit eine wichtige Rolle. Eine Schlussfolgerung lautet, dass eine gelungene Kommunikation nur in enger Zusammenarbeit möglich ist (ebd.). Diese Abstimmung zwischen Bund und Ländern hat während der Übung - nach Selbstdarstellung - gut funktioniert (Bundesamt für Bevölkerungsschutz und Katastrophenhilfe 2008). Allein die personellen Engpässe für die Presse- und Öffentlichkeitsarbeit, die über mehrere Wochen andauern würden, wurden als 
besondere Herausforderung empfunden (Bundesministerium des Innern \& Bundesministerium für Gesundheit 2007).

\section{Auswertungsberichte der LÜKEX}

Bis zum Ende der systematischen Recherche nach Dokumenten (21.02.2013) war lediglich ein Dokument im Umfang von sieben Seiten über die LÜKEX 2007 frei zugänglich (Bundesministerium des Innern \& Bundesministerium für Gesundheit 2007). Auswertungsberichte der anderen LÜKEX-Übungen (2004, $2005,2009,2011$ ) waren nur für den internen Gebrauch zugänglich und konnten trotz mehrmaliger Nachfrage nicht bezogen werden. Nach der Analyse der Dokumente wurden offizielle Auswertungsberichte zur LÜKEX 2011 und zur LÜKEX 2013 öffentlich zugänglich (Bundesamt für Bevölkerungsschutz und Katastrophenhilfe 2012a; 2014c). Da diese Berichte Nova zu der bisherigen Auswertungspraxis darstellen, werden sie an dieser Stelle zusammenfassend dargelegt.

Das der LÜKEX 2011 zugrunde liegende Szenario ging von Angriffen auf ITSysteme in Regierungsnetzwerken aus, das Szenario 2013 von einer außergewöhnlichen biologischen Bedrohungslage, die in ihrer Form der EHECEpidemie ähnelte, als Unterschied jedoch eine absichtliche Kontaminierung von Lebensmitteln annahm. Beide Auswertungsberichte sind mit 44 und 68 Seiten recht umfangreich und visuell ansprechend aufbereitet. Jeweils vier Seiten beschäftigen sich explizit mit Themen der Kommunikation (Bundesamt für Bevölkerungsschutz und Katastrophenhilfe 2012a; 2014c).

Insgesamt ist festzustellen, dass beide Berichte die LÜKEX-Übung sowie Aspekte der Krisenkommunikation grundsätzlich sehr positiv bewerten und dafür eine ebenso positive Sprache verwenden. So werden etwa die Abstimmungsprozesse zwischen den Bundesbehörden in der Übung 2013 gelobt (Bundesamt für Bevölkerungsschutz und Katastrophenhilfe 2014c). Nur vereinzelt wird Kritik deutlich. So heißt es zum Beispiel:

„Die Abstimmung der Medien- und Öffentlichkeitsarbeit zwischen den Ländern und dem Bund beziehungsweise den Bundesressorts mit dem Ziel einer einheitlichen, widerspruchsfreien Kommunikation bleibt eine Herausforderung. " (ebd.: 30)

Im Bericht über die LÜKEX 2013 werden Empfehlungen für die Krisenkommunikation abgeleitet. Eine davon betrifft die Prüfung, ob eine zentrale Informationsstelle auf Bundesebene eingerichtet werden kann, die Informationen bündelt und zielgruppenspezifisch aufbereitet. Jedoch wird diese für die Kommunikation 
mit Bürgern, nicht mit Journalisten, empfohlen. Eine weitere Empfehlung betrifft die Kommunikation von Unsicherheiten und Nichtwissen (Bundesamt für Bevölkerungsschutz und Katastrophenhilfe 2014c). Beide Berichte enthalten zudem Empfehlungen, wie Online-Medien und Social Media für behördliche Risiko- und Krisenkommunikation genutzt werden können und es wird ebenfalls erwähnt, dass die real stattfindende Medienarbeit auf große Resonanz stieß (Bundesamt für Bevölkerungsschutz und Katastrophenhilfe 2012a; 2014c) ${ }^{32}$.

\subsubsection{Interorganisationale Risiko- und Krisenkommunikation}

Nachfolgend wird gezeigt, welche Rolle interorganisationale Risiko- und Krisenkommunikation von Behörden, inklusive der OVP, in den Dokumenten spielen, auch bei Krisen im Gesundheitsbereich.

\subsubsection{Zusammenarbeit}

Eine gelungene Zusammenarbeit und die dazu bereits im Vorfeld festgelegte interorganisationale Kommunikation zwischen den Behörden gelten in der Krise als Notwendigkeit und Herausforderung zugleich, gerade wenn unterschiedliche Organisationsformen und Strukturen aufeinandertreffen (Bundesministerium des Innern 2008; Bundesamt für Bevölkerungsschutz und Katastrophenhilfe \& Deutscher Städtetag 2010). Deswegen wird konstatiert, dass die Klärung von Zuständigkeiten bereits vor einer Krise erfolgen sollte. Hierzu zählen der Aufbau und die Nutzung persönlicher Netzwerke, regelmäßige Treffen, das Kennen der Kommunikationsverantwortlichen, aktuelle Verzeichnisse sowie das Festlegen von Führungsaufgaben. Nur mit solchen Vorbereitungsmaßnahmen kann auch die zwischenbehördliche und darauf aufbauend die externe Kommunikation funktionieren (Bundesministerium des Innern 2008). Krisenübungen wie die LÜKEX werden vorgeschlagen, um Zusammenarbeit bereits in Nicht-Krisenzeiten zu üben (Bundesministerium des Innern 2014).

Es wird dargelegt, dass die Zusammenarbeit bei der Risiko- und Krisenkommunikation zwischen Bund, Ländern und Kommunen auf allen Ebenen intensiviert werden sollte (Bundesamt für Bevölkerungsschutz und Katastrophenhilfe \& Deutscher Städtetag 2010). Für ein besseres, Ebenen übergreifendes Krisenmanagement wurde deNIS II plus entwickelt. Die digitale Kommunikations-

\footnotetext{
${ }^{32}$ Darüber hinaus hat der Krisenstab der Landesregierung in Hessen, das als intensivübendes Bundesland an der LÜKEX 2011 teilgenommen hat, einen eigenen, umfangreichen Erfahrungsbericht veröffentlicht. Dieser umfasst 61 Seiten und kommt ebenfalls zu positiven, obgleich auch kritischen Bewertungen der Übung (Hessisches Ministerium des Innern und für Sport 2012).
} 
plattform soll das Krisen- und Informationsmanagement zwischen Bund, Ländern und Hilfsorganisationen unterstützen (Seiwert et al. 2007).

\subsubsection{One-voice-policy}

In neun B-Dokumenten wird die One-voice-policy als besonders wichtig, teilweise als unentbehrlich verstanden und mehrfach betont (z. B. Bundesministerium des Innern 2008; Bundesamt für Bevölkerungsschutz und Katastrophenhilfe 2011b; Deutscher Städtetag 2012). Unter One-voice-policy werden einheitliche Sprachregelungen von verschiedenen Akteuren verstanden. Dazu zählen die regelmäßige Abstimmung und die Festlegung von Maßnahmen und Zuständigkeiten für den Umgang mit Medien und für offizielle Veröffentlichungen (Robert Koch-Institut 2007; Bundesministerium des Innern 2008; Bundesamt für Bevölkerungsschutz und Katastrophenhilfe 2011d; Deutscher Städtetag 2012).

Meinungsäußerungen von zu vielen Stimmen werden demnach als „schlechte Kommunikation" (Deutscher Städtetag 2012:17) gewertet. Unterschiedliche Informationen von verschiedenen Behörden zum gleichen Sachverhalt würden Verwirrung und Vertrauensverluste in der Bevölkerung auslösen. Es wird empfohlen, ,Kompetenz- und Zuständigkeitsgerangel “ (Bundesministerium des Innern 2008: 24) in der Öffentlichkeit zu vermeiden.

\subsubsection{Interorganisationale Kommunikation bei Krisen im Gesundheitsbereich}

Als Voraussetzung einer gelungenen Krisenkommunikation während einer Pandemie gilt eine einheitliche Kommunikation zwischen den Akteuren des Gesundheitssystems auf allen Ebenen (Kommunal-, Landes- und Bundesebene, internationale Ebene) (Robert Koch-Institut 2007; 2015). Dies umfasst den Aufbau und die Etablierung zwischenbehördlicher Risikokommunikation bereits vor einer Krise (Robert Koch-Institut 2015). Die Bedeutung der zwischenbehördlichen Risiko- und Krisenkommunikation ist in dem aktuellen Pandemieplan zwar erkennbar, jedoch erfolgen keine konkreten Ausführungen darüber, wie diese gestaltet sein sollte. Recht allgemein wird der mögliche Einsatz von Krisenstäben und Lagezentren auf Bundes- oder Landesebene in der Krise thematisiert, um Entscheidungen auf fachlicher und politischer Ebene schnell abzustimmen und nach außen zu kommunizieren. Potentielle Herausforderungen werden nicht thematisiert (ebd.). Die Vermittlung konsistenter, widerspruchfreier Botschaften im Sinne einer One-voice-policy wird auch für den Fall einer Influenzapandemie als wichtig erachtet (Robert Koch-Institut 2007; 2015; Bundesamt für Bevölkerungsschutz und Katastrophenhilfe 2011c). 


\section{Epidemisches Infektionsgeschehen}

Im September 2013 wurde die „Allgemeine Verwaltungsvorschrift über die Koordinierung des Infektionsschutzes in epidemisch bedeutsamen Fällen" der Bundesregierung veröffentlicht (Die Bundesregierung 2013). Sie wurde als Folge der EHEC-Epidemie erarbeitet. Aufgrund des Erscheinungsdatums konnte dieses Dokument nicht in der systematischen Auswertung berücksichtigt werden. $\mathrm{Da}$ die Verwaltungsvorschrift für den Untersuchungsgegenstand jedoch neue Aspekte enthält, wurde sie als Nachzügler-Dokument einbezogen.

Die Verwaltungsvorschrift verfolgt das Ziel Erfahrungen, die bei bisherigen Krankheitsausbrüchen gemacht wurden, stärker für Abstimmungsprozesse zwischen Bund und Ländern zu berücksichtigen. Sie möchte Aspekte der Zusammenarbeit während einer epidemisch bedeutsamen Krise festlegen. Das Dokument beinhaltet Ausführungen über Zuständigkeiten für Informationsaufgaben sowie Abläufe für die zwischenbehördliche Kommunikation. Es wird deutlich, dass dem RKI dabei eine Schlüsselposition zukommt. Es ist für die Koordinierung der Kommunikationsprozesse zwischen den Behörden sowie die Informationsbereitstellung für diese verantwortlich (ebd.).

Für Informationen über wissenschaftliche Fragen sowie über die länderübergreifende und internationale Entwicklung der Situation ist der Bund zuständig. Bundesländer und Kommunen sollen darüber hinaus regionale Spezifika in der Kommunikation ergänzen. Es wird ausgeführt, dass eine Behörde eines Bundeslandes oder einer Kommune eine Information, die von den bundesweit kommunizierten Inhalten abweicht oder wesentliche Neuigkeiten enthält, zunächst an das RKI für eine fachliche Beurteilung senden soll (ebd.). Dieser Absatz ist vermutlich auf das Vorgehen der Hamburger Behörde für Gesundheit und Verbraucherschutz während der EHEC-Epidemie zurückzuführen, als diese - ohne es mit anderen Behörden abzustimmen - behauptet hatte, dass Gurken spanischer Unternehmen die Ursache für das Bakterium seien, was sich später als falsch herausstellte.

In der Verwaltungsvorschrift wird außerdem dargelegt, dass auf Unsicherheiten sowie unvollständige Informationen hinzuweisen ist. Das RKI soll Medien auch dann informieren, wenn es eventuell keine neuen Kenntnisse gibt, das mediale Interesse aber unverändert hoch ist (ebd.). Dies entspricht einer kontinuierlichen Kommunikation.

Wenn es im Verlauf von mehreren Jahren nicht zu einem epidemisch bedeutsamen Fall kommt, soll die Koordinierung der Presse- und Öffentlichkeitsarbeit 
geübt werden (Die Bundesregierung 2013). Dieser Zeitraum wird jedoch nicht spezifiziert.

\subsubsection{Interaktionspartner Medien}

\subsubsection{Thematisierung der medialen Logik bei Risiken und Krisen}

Die mediale Logik in Bezug auf Risiken wird nur marginal in zwei Dokumenten im Kontext der Risikowahrnehmung und Risikovermittlung thematisiert (Schröder 2011; Böl 2013). In vier Dokumenten wird die mediale Logik während einer Krise thematisiert, um Verantwortlichen für Krisenkommunikation Wissen über journalistische Arbeitsweisen zu vermitteln (Bundesministerium des Innern 2008; 2014; Bundesamt für Bevölkerungsschutz und Katastrophenhilfe 2011b; Schröder 2011; Deutscher Städtetag 2012).

Es wird dargelegt, dass eine Krise in kurzer Zeit ein verstärktes Medieninteresse und eine erhöhte Aufmerksamkeit bewirkt, wodurch mit vermehrten - auch kritischen - Anfragen von Journalisten zu rechnen ist (Bundesamt für Bevölkerungsschutz und Katastrophenhilfe 2011b). Dadurch kann eine Eigendynamik entstehen, die sich wesentlich schneller entwickelt, als die Behörden darauf reagieren können (Deutscher Städtetag 2012). In einem Dokument wird nach drei Phasen der Krisenberichterstattung während einer Krise differenziert (Bundesministerium des Innern 2008).

Es wird ausgeführt, dass Medien und Journalisten ihre eigenen Interessen und Arbeitsweisen verfolgen, bei denen allein das Publikum im Fokus steht. Aus diesem Grund ist die Berichterstattung nur teilweise seriös (Deutscher Städtetag 2012). In einem anderen Dokument wird darüber hinaus konstatiert, dass Medien sich zum Teil als „vierte Macht" (Bundesministerium des Innern 2008: 24; 2014: 19) betrachten, weshalb sie eventuelle Missstände in den Behörden aufzudecken versuchen. Auch dieses Interesse kann - insbesondere in Krisen zu überzogenen Berichten führen (Bundesministerium des Innern 2008). Ferner wird dargelegt, dass Informationen schneller, direkter, kompromissloser und nicht immer wahrheitsgetreu verbreitet werden, woraus spekulative Äußerungen oder Skandalisierungen resultieren können (Schröder 2011; Deutscher Städtetag 2012).

Aufgrund der sich verändernden Medienwelt werden Informationen nahezu in Echtzeit publiziert, was eine immense Herausforderung für die Krisenkommunikation darstellt (vgl. Kap. 8.1.3.6). Es wird zudem dargelegt, dass ein NichtKommunizieren eines verantwortlichen Akteurs dazu führen kann, dass Journa- 
listen krisenrelevante Informationen aus anderen Quellen beziehen oder spekulieren (Deutscher Städtetag 2012).

\subsubsection{Empfehlungen zum Umgang mit Medien in der Krise}

In Kapitel 8.1.3.5 wurden bereits zahlreiche Empfehlungen aufgeführt, die auch für die externe Krisenkommunikation mit Medien gelten. Nachfolgend werden deshalb vor allem Dinge aufgezeigt, die in dieser Form noch keine Erwähnung fanden.

Ein Dokument führt aus, dass Behörden von Medien abhängig sind, da sie in einer Krise einerseits die Nachrichten, die Medien verbreiten, zu ihrer eigenen Informationslage benötigen und andererseits die Zielgruppe Bevölkerung nur über Medien großflächig erreichen (Bundesministerium des Innern 2008). Entsprechend wird Behörden empfohlen, Journalisten und ihren Arbeitsweisen in Krisen besondere Aufmerksamkeit entgegenzubringen (Bundesamt für Verbraucherschutz und Lebensmittelsicherheit 2006).

Für den Umgang mit Medien und Journalisten in einer Krise werden die frühzeitige Vorbereitung (auch von Textbausteinen), die Regelung von Zuständigkeiten, die Etablierung und Pflege eines festen Journalistennetzwerkes, die kontinuierliche Informierung von Journalisten sowie ein gegenseitiges Vertrauensverhältnis als bedeutend aufgeführt (z. B. Bundesministerium des Innern 2008; Bundesamt für Bevölkerungsschutz und Katastrophenhilfe \& Deutscher Städtetag 2010; Bundesamt für Bevölkerungsschutz und Katastrophenhilfe 2011d). Nur durch einen regelmäßigen Austausch kann ein Vertrauensverhältnis zwischen Medienund Behördenmitarbeitern hergestellt werden. Dieses Vertrauensverhältnis gilt als essentiell, um eine gelungene Kommunikation während einer Krise zu ermöglichen und eventuelle Gerüchte und Spekulationen zu vermeiden (Bundesministerium des Innern 2008; Bundesamt für Bevölkerungsschutz und Katastrophenhilfe \& Deutscher Städtetag 2010).

Außerdem werden einheitliche Sprachregelungen im Sinne einer One-voicepolicy, spezielle Medientrainings für Mitarbeiter der Pressestelle sowie ggf. externe Unterstützung durch Kommunikationsspezialisten empfohlen (Bundesministerium des Innern 2008; Bundesamt für Bevölkerungsschutz und Katastrophenhilfe 2011d). Es wird mehrfach geäußert, dass die Pressestelle der jeweiligen Behörde für die Kommunikation mit Medien verantwortlich ist (Bundesamt für Verbraucherschutz und Lebensmittelsicherheit 2006; Bundesamt für Bevölkerungsschutz und Katastrophenhilfe 2011d; Deutscher Städtetag 2012). Gleichzeitig wird empfohlen, dass hochrangige Mitarbeiter einer Behörde als Sprecher agieren sollten (vgl. Kap. 8.1.3.5). 
$\mathrm{Zu}$ journalistischen Erwartungen an die behördliche Risiko- und Krisenkommunikation liegen, bis auf die allgemeine Empfehlung die Anforderungen von Medien zu berücksichtigen, keine Aussagen in den Dokumenten vor.

\subsubsection{Risiken Lebensmittelengpass, Influenzapandemie}

In der Mehrzahl der Dokumente werden keine spezifischen Risiken oder mögliche Krisenszenarien erwähnt. In vier Dokumenten werden Szenarien allgemein erwähnt, etwa wie bedeutend die Entwicklung von Szenarien für eine groß angelegte Krisenmanagement-Übung ist (Bundesamt für Bevölkerungschutz und Katastrophenhilfe 2011b; Weber \& Dieker 2012). In einem Dokument werden potentielle Krisen aufgelistet, darunter auch Pandemien (Weber 2013).

In einem E-Dokument wird ENV thematisiert. Es wird eine private Bevorratung mit Lebensmitteln empfohlen sowie auf das Portal www.ernaehrungsvorsorge.de verwiesen (Baach \& Burmeister 2012). Außerdem werden gesundheitliche Szenarien wie eine Influenzapandemie oder ein Pockenausbruch als Beispiele schwerer Krisen aufgeführt. In diesem Zusammenhang wird lediglich erwähnt, dass die Risikokommunikation mit der Öffentlichkeit eine Rolle spielt (ebd.).

Im Vergleich zu einem Lebensmittelengpass wird der Influenzapandemie in den Dokumenten eine höhere Bedeutung zugeschrieben. So setzen sich zwei B-Dokumente und ein E-Dokument ausschließlich mit diesem Risiko auseinander (Bundesamt für Bevölkerungsschutz und Katastrophenhilfe \& Robert Koch-Institut 2007; Robert Koch-Institut 2007; 2014; 2015; Bundesamt für Bevölkerungsschutz und Katastrophenhilfe \& Landesgesundheitsamt BadenWürttemberg 2010). Die verhältnismäßig hohe Bedeutungszuschreibung lässt sich auch daran erkennen, dass die LÜKEX 2007 eine Influenzapandemie als Szenario für die Übung zugrunde legte (vgl. Kap. 8.1.5).

Für die systematische Auswertung der Dokumente wurde der Nationale Pandemieplan aus dem Jahr 2007 berücksichtigt. Mit Abschluss der Recherche nach Dokumenten (21.02.2013) und damit nach der H1N1-Influenza in den Jahren 2009/2010 lag noch immer lediglich der Pandemieplan von 2007 vor. Nicht zuletzt in den durchgeführten Experteninterviews mit Behörden wurde verdeutlicht, dass es einer Überarbeitung des Pandemieplans bedarf (vgl. Kap. 8.2.5.1). Ende 2014 sind die ersten Teile eines neuen Pandemieplans erschienen, darunter Teile über die Themen „Epidemiologie“, „Risikoeinschätzung“, „Surveillance“ sowie eine Einleitung. Im September 2015 wurden weitere Teile über „Impfstoffkonzepte“, „Pandemierelevante Arzneimittel“, „Virologische Grundlagen“ und über „Kommunikation“ veröffentlicht. Nachdem der Nationale Pandemieplan zuvor 
aus vier Teilen bestand, umfasst er nun acht einzelne, thematische Teile (Robert Koch-Institut 2015).

In dem Plan von 2007 sind die empfohlenen Kommunikationsmaßnahmen und -handlungen noch sehr allgemein gehalten, das hat sich mit dem Plan von 2015 verändert. Dieser thematisiert detaillierter Voraussetzungen, Maßnahmen sowie Empfehlungen zur Durchführung von Risiko- und Krisenkommunikation während eines pandemischen Geschehens (ebd.).

Im neuen Pandemieplan wird zwischen zwei grundlegenden Richtungen der Kommunikation differenziert, von der die erfolgreiche Bewältigung pandemischer Geschehnisse abhängt: der zwischenbehördlichen Kommunikation und der externen Kommunikation gegenüber Fachöffentlichkeit, Medien und Bevölkerung. Die Ausführungen widmen sich insbesondere Kommunikationsmaßnahmen gegenüber Bevölkerung und Medien, jedoch kommt Medien im Vergleich zur Bevölkerung eine nur untergeordnete Relevanz als Zielgruppe zu (ebd.). Da sich ein pandemisches Geschehen tendenziell nicht regional einheitlich entwickelt, wird die Wichtigkeit von regionalen, lagespezifischen Krisenkommunikationsstrategien betont, die an die Situation angepasst werden und flexibel sein müssen (ebd.). 


\subsection{Risiko- und Krisenkommunikation von Behörden: Ergebnisse der Experteninterviews}

\subsubsection{Strukturen von Risiko- und Krisenkommunikation}

Risiko- und Krisenkommunikation sind in den befragten Behörden nicht organisational verankert, z. B. in Form einer Abteilung oder verantwortlichen Person. Eine Behörde stellt eine Ausnahme dar. Der Umgang mit Risiko- und Krisenkommunikation gestaltet sich strukturell unterschiedlich.

In der Regel gibt es bei den Behörden eine Pressestelle, die bestimmte Themen, darunter teilweise auch Risiken, kommuniziert respektive in Krisen kommunizieren würde. Je nach Thema wird dafür Input aus den Fachreferaten herangezogen. Es lässt sich feststellen, dass Risikokommunikation, insofern diese stattfindet, insbesondere durch die von den Ministerien nachgeordneten Bundesoberbehörden ausgeführt wird.

In zwei der befragten Behörden existiert ein eigenes Referat für Krisenmanagement. Von einigen Behörden wird ausgeführt, dass sich in Krisen ein Krisenstab - entweder hausintern oder gemeinsam mit Ministerien, nachgeordneten Bundesbehörden oder Bundesländern - konstituieren würde. Dabei lassen sich keine einheitlichen Handlungsmuster in Bezug darauf identifizieren, welche Akteure beteiligt wären oder ab welchem Ausmaß einer Krise sich der Krisenstab konstituieren würde. Von einer Behörde wird dargelegt, dass sie bereits des Öfteren Erfahrungen mit Krisenkommunikation, besonders im Bereich der Lebensmittelsicherheit, sammeln musste (z. B. BSE, EHEC-Epidemie, mit Dioxin oder Noroviren kontaminierte Lebensmittel).

\subsubsection{Risikokommunikation}

\subsubsection{Verständnisse von Risikokommunikation}

Bei drei Interviewpartnern ist ein dialogisches und partizipatives Verständnis von Risikokommunikation im Sinne eines wechselseitigen Kommunikationsprozesses über Risiken, inklusive der Einbindung von Anspruchsgruppen, zu identifizieren (E1; E6; E8). In einer dieser Behörden wird Risikokommunikation als „das Übersetzen von Wissenschaft für alle interessierten Gruppen“ (E1: A110) verstanden, um diese einzubeziehen. Einweg-Kommunikation, z. B. in Form von Flyern, sollte nicht die einzige Form von Risikokommunikation sein (E1). Zugleich wird ausgeführt, dass die Umsetzung eines solchen wechselseitigen Prozesses in der Praxis sehr schwierig ist (E8) sowie dass die Behörde 
eines Experten Risikokommunikation in dieser Form nicht umsetzt und lediglich einseitig kommuniziert (E6). Risikokommunikation wird von zwei anderen Behörden mit einem Informationsprozess und der Sensibilisierung für bestimmte Risiken in Verbindung gebracht. Dabei wird Risikokommunikation eng gekoppelt an präventive Handlungen auslösende Botschaften betrachtet (E7; E9).

Nicht aus allen Interviews lässt sich ein eindeutiges Verständnis von Risikokommunikation ableiten. Zwei Behörden verwenden den Begriff nicht oder sehen Risikokommunikation nicht in ihrem Aufgabenbereich (E2; E4). Außerdem wird es als ein theoretischer Diskurs erachtet, was unter Risiko- oder Krisenkommunikation verstanden wird (E2).

\subsubsection{Ziele und Zielgruppen von Risikokommunikation}

Folgende Ziele der Risikokommunikation werden mit entsprechenden Häufigkeiten genannt:

- Vertrauen in der Bevölkerung aufbauen (3x)

- Bevölkerung befähigen, mit einem Risiko umzugehen und für Risiken Vorsorge zu betreiben (3x)

- $\quad$ Für Risiken bei verschiedenen Zielgruppen sensibilisieren (3x)

- Bürger in Risikokommunikationsprozesse einbeziehen (2x)

- $\quad$ Sachlich und neutral aufklären, informieren (1x)

- $\quad$ Auf mögliche Krisen vorbereiten (1x)

- $\quad$ Langfristige Kommunikation etablieren (1x)

- Unbegründete Ängste vermeiden (1x)

- $\quad$ Lehren, die aus Krisen(kommunikation) gezogen werden, in Risikokommunikation berücksichtigen (1x)

Als Zielgruppen von Risikokommunikation werden von den Experten genannt:

- $\quad$ Bürger (6x)

- $\quad$ Medien (6x)

- $\quad$ Staatliche Organisationen (Bundes- und Landesebene) (4x)

- Unternehmen $(2 \mathrm{x})$

- Multiplikatoren, z. B. Lehrer (1x)

- $\quad$ NGOs (1x)

- Wissenschaft $(1 \mathrm{x})$

- $\quad$ Fachöffentlichkeiten (1x)

- $\quad$ Mitarbeiter (1x) 


\subsubsection{Instrumente von Risikokommunikation}

Als Instrumente der Risikokommunikation werden genannt:

- Maßnahmen der Pressearbeit (5x)

- Websites (3x)

- FAQs (3x)

- $\quad$ Besuch von Fachmessen (3x)

- $\quad$ Broschüren $(2 x)$

- Informationssystem Ernährungsnotfallvorsorge (IS ENV) (2x)

- Bürger- bzw. Verbrauchertelefon (2x)

- Beantworten von Anfragen (2x)

- Kampagnen (1x)

- $\quad$ LÜKEX $(1 \mathrm{x})$

- Werbliche Maßnahmen wie Anzeigen, Kino- und Fernsehspots (1x)

- Monitoring im Gemeinsamen Melde- und Lagezentrum (GMLZ) (1x)

- Risikobewertungen/Stellungnahmen (1x)

- Wissenschaftliche Veranstaltungen, z. B. Verbraucherkonferenzen (1x)

- $\quad$ Studien $(1 \mathrm{x})$

- Zeitschriften $(1 \mathrm{x})$

Zum Zeitpunkt der Befragung sind die Social-Media-Aktivitäten der Behörden als insgesamt zurückhaltend zu kennzeichnen. Bei drei Behörden heißt es, dass Social Media derzeit aufgebaut und zukünftig - auch in Krisen - eine Rolle spielen werden (E4; E7; E8). Man hofft, mit Social Media sowie onlinevermittelter Kommunikation allgemein, mehr Menschen kostengünstig(er) zu erreichen (E8).

Diese Erkenntnisse müssen im zeitlichen Kontext der Interviews gesehen werden, die 2013 geführt wurden. Inzwischen pflegen sechs der acht befragten Behörden einen eigenen Twitter-Auftritt, sechs einen eigenen YouTube-Auftritt sowie eine Behörde einen eigenen Facebook-Auftritt (Stand: Dezember 2015). Dies, die Ergebnisse der Dokumentenanalyse sowie die in den Interviews getätigten Aussagen über die zukünftige Planung zeigen, dass Entwicklungen in diesem Bereich stattfinden. Gleichwohl besteht, insbesondere bei der Nutzung von Facebook, noch immer große Zurückhaltung. Gründe, die aus Sicht der Behörden gegen eine Social-Media-Nutzung für Risiko- und Krisenkommunikation sprechen, sind verbraucherschutzpolitische Bedenken, mangelnde Kapazitäten in der Krise, eine nur schwer zu kontrollierende Dynamik sowie dass man Journalisten nicht zu viele Kanäle „zumuten“ möchte (E1; E5; E6; E9) (vgl. hierfür auch Drews \& Raupp 2016). Aufgrund der Befunde muss zusätzlich hinterfragt werden, inwiefern Social-Media-Kanäle tatsächlich als dialogische 
Kommunikationsinstrumente und nicht lediglich als zusätzliche Distributionskanäle verwendet werden.

\subsubsection{Herausforderungen der Risikokommunikation}

Eine besondere Herausforderung der Risikokommunikation sehen zwei Interviewpartner darin, zu sensibilisieren ohne die Bevölkerung zu verängstigen oder Panik zu schüren (E7; E8). Für eine Behörde besteht eine Herausforderung vor allem in der Vermittlung von Inhalten ohne konkrete Anlässe, also in Zeiten, in denen meist kein öffentliches Interesse an bestimmten Risiken existiert. Dafür neue Wege zu finden, wird als schwierig beurteilt. Damit geht auch eine gewisse Langwierigkeit von Risikokommunikationsprozessen einher (E8).

\subsubsection{Krisenkommunikation}

\subsubsection{Verständnisse von Krisen}

Es zeigen sich zwei grundsätzliche Verständnisse von Krise: ein kommunikatives und ein rechtliches. Ersteres betrifft das Verständnis von Krise als ein außergewöhnlich hohes Kommunikationsaufkommen zu einem Thema, das viel mediale Aufmerksamkeit nach sich zieht (E5; E7). Eine Krise ist demnach ,eine besonders zugespitzte Lage mit einer Vielzahl von Medienanfragen, mit einer hohen öffentlichen Awareness, oft begleitet von einem Meinungskampf in den Medien "(E5: A84).

Das rechtliche Verständnis bezeichnet eine Krise als eine Gefahren- und Schadenslage für die innere Sicherheit mit schweren Auswirkungen. Demnach liegt erst dann eine Krise vor, wenn sich ein Krisenstab konstituieren muss (E7). Im Sinne dieses rechtlichen Verständnisses vertreten vier Interviewpartner die Meinung, dass es eine Krise in Deutschland bislang noch nicht gegeben hat (E3; E5; E6; E7). Darüber hinaus erfolgt eine Differenzierung nach politischen Krisen (z. B. die „Dienstwagenaffäre“ von Ulla Schmidt) und „tatsächlichen“ Krisen, in denen die Bevölkerung bedroht ist (z. B. EHEC-Epidemie, Erkrankung von Schulkindern mit Noroviren) (E3; E4; E5).

\subsubsection{Strategische Elemente der Krisenkommunikation}

Um die Bedeutung von Krisenkommunikation in den Behörden zu eruieren, wurde nach strategischen Elementen gefragt. Diese umfassen Zielgruppen, Kommunikationsinstrumente, vorliegende strategische Konzepte sowie Empfehlungen für Krisenkommunikation. 
Die Zielgruppen von Krisenkommunikation konnten nur ungenau bestimmt werden, unter anderem aufgrund der Äußerungen einiger Interviewpartner, noch nie Krisenkommunikation durchgeführt zu haben. Es ist jedoch davon auszugehen, dass die Zielgruppen der Krisenkommunikation weitestgehend denen der Risikokommunikation entsprechen (vgl. Kap. 8.1.2.2, 8.2.2.2).

Die Instrumente, die bislang für Krisenkommunikation verwendet wurden, überschneiden sich größtenteils mit denen der Risikokommunikation. Zusätzlich wird die Bedeutung folgender Kommunikationsinstrumente für die Krise betont: Krisenhotline (4x), FAQs (3x), Themenwebsite (2x) und Maßnahmen der Presseund Öffentlichkeitsarbeit (alle Experten). Im Kontext der Pressemaßnahmen wird erwähnt, dass in der Krise Möglichkeiten zur Verfügung gestellt werden sollten, damit Journalisten Ton- und Videoaufnahmen machen können (E2). Die Thematik von Social Media in der Krisenkommunikation wurde bereits im Rahmen der Risikokommunikation ausgeführt. Zum Zeitpunkt der Befragung wurden noch keine Social-Media-Kanäle in Krisen genutzt.

Hinsichtlich einer Strategie oder festen Vorgehensweise für eine Krise verweisen einige Experten auf vorliegende Konzepte, Telefon- oder Zuständigkeitslisten (E4; E7; E8) sowie auf Grundregeln, die in der Krise beachtet werden sollten (E5). Eine Behörde beruft sich darauf, dass eine Krise immer ähnlich ablaufen würde und man sich mit dem übergeordneten Ministerium abstimmen, gegenseitig informieren und entsprechend der Vorgaben des Ministeriums agieren würde. Im Gegensatz dazu führt ein Interviewpartner aus, dass keine Krise wie die andere ist, weshalb es auch keine ,Standardmuster" (E5: A90) der Bewältigung gibt. Es existiert jedoch ein genereller Instrumentenkasten, aus dem in der Krise ausgewählt wird (E5).

Als Empfehlungen für die Krisenkommunikation werden folgende genannt:

- Transparent und ehrlich kommunizieren (3x)

- $\quad$ Korrekte Fakten kommunizieren (2x)

- Unsicherheiten kommunizieren (2x)

- $\quad$ Nicht spekulieren, vermuten oder Schuldfragen diskutieren (2x)

- Interne Strukturen, Abläufe und Ansprechpartner kennen (2x)

- Zuständigkeiten klären $(2 \mathrm{x})$

- Informationen sammeln, Lagebild erstellen (2x)

- $\quad$ Flexibel sein $(2 x)$

- $\quad$ Über erfahrenes, kompetentes Personal verfügen (2x)

- $\quad$ Kontinuität hinsichtlich der personellen Besetzung herstellen (1x)

- Neutral kommunizieren (1x) 
- Informationen schnell aufbereiten (1x)

- Gemeinsames, abgestimmtes Verhalten (1x)

- Vorhandensein einer Strategie (1x)

- Krisenkommunikation als „Chefsache“ betrachten $(1 \mathrm{x})$

- $\quad$ Flache Hierarchien (1x)

- Permanente Besetzung und Erreichbarkeit der Pressestelle, auch am

Wochenende und in der Nacht (1x) (wird gleichermaßen als

Herausforderung angesprochen, vgl. nachfolgende Ausführungen)

\subsubsection{Herausforderungen der Krisenkommunikation}

Nachfolgend werden die zentralen Herausforderungen der Krisenkommunikation aus Sicht der Behörden dargelegt.

Finanzielle, personelle und zeitliche Ressourcen:

Krisenkommunikation stellt für die Behörden eine personelle und zeitliche Belastung dar.

„Es ist dann 7 Tage die Woche von 6 Uhr bis 23 Uhr, da gibt es auch keine Rücksichtnahme auf Arbeitszeiten oder sowas. Da wird ständig erwartet, ständiges Update, natürlich wird auch im Internet erwartet, dass alles immer aktuell ist. “(E5: A90)

Dafür benötigt man eine vielfache Zahl an Mitarbeitern (E2; E5; E7). Ein Interviewpartner äußert jedoch, dass die Behörde nicht einen beliebig großen Kommunikationsstab aufbauen kann, weil es permanent an Personal und Geldern mangelt (E2). Zudem herrscht in Ministerien eine hohe Fluktuation des Personals auf Leitungsebene, was auch Pressesprecher betrifft. Diese fehlende Kontinuität ist eine Schwierigkeit hinsichtlich kontinuierlicher Kommunikationsbemühungen (E2).

Es wird kritisiert, dass politische Akteure in Krisen schnell behaupten, mehr Geld in die jeweilige von der Krise betroffene Infrastruktur stecken zu wollen (z. B. bei Hochwasser), sobald die Krise aber in Vergessenheit geraten ist, nicht mehr die Notwendigkeit für mehr finanzielle Unterstützung gesehen wird. In diesem Zusammenhang wird von einer Behörde auf die eigenen begrenzten finanziellen Möglichkeiten verwiesen (E8).

Strukturen von Behörden:

Zwei Interviewpartner führen aus, dass Behörden oft hierarchischen Strukturen und Abläufen unterliegen, die schnelle Entscheidungen erschweren. Für die sehr langsamen oder manchmal gar nicht stattfindenden Abstimmungen zwischen den 
Behörden und den daraus resultierenden Äußerungen haben Medien oft nur wenig Verständnis, was insbesondere in der Krise, wenn Informationen noch schneller als sonst verfügbar sein müssen, eine Schwierigkeit darstellt. Diese Langsamkeit und Vorsicht wird mit der Angst etwas Falsches zu sagen begründet, da man als Behörde eine besondere Verantwortung trägt (E2; E8). Zudem wird es als Herausforderung betrachtet, dass es einen Ressortfokus gibt und sich ein Ministerium in der Regel nicht für die Handlungen anderer Ministerien interessiert (E2).

\section{Zusammenarbeit zwischen Behörden:}

Es wird geäußert, wie schwierig die operative Zusammenarbeit zwischen Fachbehörden und Ministerien ist. Dies liegt an hierarchischen Strukturen, mangelndem Vertrauen zueinander sowie daran, dass ein Ministerium andere Ziele verfolgt als eine Fachbehörde. Hierbei handelt es sich vor allem um politische Interessen. Aus Sicht dieses Experten bedeuten diese unterschiedlichen Selbstverständnisse, dass Krisen, die gut bewältigt werden können, eher für politische Zwecke genutzt werden und in bedrohlichen Krisen, in denen es viele Todesfälle gibt, eher versucht wird, die Zuständigkeiten an andere abzutreten (E2). Ein anderer Interviewpartner vermutet jedoch, dass „,mildere“ Formen der Krise eher für den politischen Meinungskampf zwischen Regierung und Opposition genutzt werden, bei einer ernsteren Lage hingegen alle Akteure disziplinierter an der Bewältigung der Krise mitwirken (E5).

\section{Abgestimmte Informationen:}

In Hinblick auf verschiedene Bundes- und Landesbehörden wird eine abgestimmte Kommunikation als besondere Herausforderung empfunden, auch wegen der Vielzahl journalistischer Anfragen. Im Zweifel müssten dann auch nicht abgestimmte Informationen veröffentlicht werden (E5).

\section{Schnelligkeit:}

Der immer schnellere Kommunikationsfluss, u. a. aufgrund von onlinevermittelter Kommunikation, erschwert die Krisenkommunikation der Behörden, da sie sich dieser Schnelligkeit anpassen müssen, was wiederum vieler Ressourcen bedarf. Gleichzeitig wächst der Anspruch von Stakeholdern, immer schneller informiert zu werden (E6; E9).

\section{Öffentlicher und medialer Druck:}

In der Krise unterliegen die involvierten Behörden Druck von vielen Seiten (E5). Mehrere Experten thematisieren den hohen Druck durch Medien, die Informationsflut und ,diese Hysterie“ (E4: A139) (E2; E3; E4). Meistens gibt es dann eine extrem hohe Zahl an Presseanfragen und mehr Fragen als Antworten (E2; E7). Zwei Interviewpartner finden es nachvollziehbar, dass es diesen öffent- 
lichen Druck gibt und Erwartungen nach Antworten und Lösungen laut werden (E2; E5).

\section{Äußerungen von ,Experten “ und Kritikern:}

Im Rahmen der Krisenberichterstattung kritisieren drei Interviewpartner „Experten“, die sich mit teils kontroversen Meinungen Gehör verschaffen möchten und so die Krisenkommunikation der Behörden erschweren (E2; E3; E4). Unter diesen Umständen ist es besonders schwierig, fachliche Informationen korrekt zu vermitteln und sachlich zu bleiben (E3; E4).

„Es gibt immer ein paar Kritiker, die man kennt, die sich immer äußern und die immer darauf achten, dass sich ihre Äußerung etwas abhebt von dem Mainstream der Behörden und der öffentlichen Stellen. “(E2: A47)

Keine Differenzierung nach Zuständigkeiten:

Es wird ausgeführt, dass Journalisten nicht nach Zuständigkeiten differenzieren, sondern erwarten, dass die angefragte Behörde in der Krise alles weiß, auch wenn die Zuständigkeiten in der Krise woanders, z. B. bei den Bundesländern, liegen (E4; E5). Dann ist es als Kommunikationsverantwortlicher schwierig, die Journalisten an die Bundesländer zu verweisen und nicht selbst Auskunft geben zu können (E5).

\section{Vermittlung von Unsicherheiten:}

Unsicherheiten zu vermitteln, wie im Fall der EHEC-Epidemie, und als Behörde zuzugeben, dass man nicht weiß, woran es liegt - insbesondere, wenn es viele Erkrankte und sogar Tote gibt - wird als sehr schwierig empfunden. In so einer Situation muss man besonders viel erklären (E5).

\subsubsection{Zusammenhängende Betrachtung}

Drei Interviewpartner äußern die Meinung, dass Risiko- und Krisenkommunikation zusammenhängen und miteinander verbunden sind (E3; E6; E7). Es werden insbesondere Gemeinsamkeiten und Unterschiede der Disziplinen herausgestellt, wobei der Fokus auf den Unterschieden liegt.

\section{Gemeinsamkeiten von Risiko- und Krisenkommunikation}

Risiko- und Krisenkommunikation haben Eigenschaften der Kommunikation wie Transparenz, Ehrlichkeit und Wahrhaftigkeit gemeinsam (E8). Eine weitere Gemeinsamkeit beider Disziplinen betrifft die strategische Ausrichtung der Kommunikation, also bereits im Vorfeld Überlegungen und Entscheidungen dazu zu 
treffen (E1). Aus Sicht eines Interviewpartners ist Risiko- und Krisenkommunikation gemein, dass beide Bestandteile des Krisenmanagements sind (E7).

\section{Unterschiede von Risiko- und Krisenkommunikation}

Als größter Unterschied zwischen Risiko- und Krisenkommunikation wird die zeitliche Ebene gesehen (E1; E2; E5; E7; E8; E9). Demnach ist Risikokommunikation ein ganzheitlicher, langfristiger Kommunikationsprozess, der für Risiken sensibilisieren, Vertrauen aufbauen und bestmöglich eine Selbstständigkeit im Umgang mit Risiken entwickeln soll. Die Krise hingegen wird als ein akutes, in sich geschlossenes Ereignis betrachtet (E2; E5; E6; E8; E9).

Risikokommunikation ist der Krisenkommunikation vorgelagert, Krisenkommunikation baut demnach auf Risikokommunikation auf (E7; E9). Aus einem Risiko kann eine Krise entstehen, weshalb Risikokommunikation auch ,prophylaktisch“ angelegt sein und Krisen - insofern möglich - verhindern sollte (E3). Risikokommunikation findet permanent, nahezu täglich, meist zu bekannten Themen statt, während Krisenkommunikation nur bei besonderen Ereignissen eine Rolle spielt (E5). Auf Themen der Risikokommunikation kann man sich einstellen oder sie proaktiv setzen, bei der Krise kann man kommunikativ meist nur reagieren, weil sie häufig ohne Vorlauf kommen (E5; E6).

Was im Risikokommunikationsprozess als erstrebenswert gilt - alle Anspruchsgruppen in den Kommunikationsprozess einzubeziehen - ist mit Krisenkommunikation kaum möglich. Dann muss man sich entscheiden, wen man adressiert, da meist keine Zeit bleibt, um partizipativ zu kommunizieren (E1).

Ein Interviewpartner führt aus, dass Krisenkommunikation als Begriff durchaus geläufiger ist als Risikokommunikation und dass beide Begriffe oft in einem Atemzug genannt werden. Eine Unterscheidung ist aber wichtig, auch wenn diese schwierig zu vermitteln ist (E8). Ein anderer Interviewpartner empfindet eine Differenzierung zwischen Risiko- und Krisenkommunikation hingegen als rein akademische Diskussion und damit ohne Relevanz für seinen Alltag (E2).

\section{Krisennachbereitung}

Als Formen der Nachbereitung einer Krise wurden bislang von vier Behörden interne Nachbesprechungen durchgeführt (E2; E4; E5; E6). Hin und wieder finden in einer Behörde externe Evaluationen über die Krisenberichterstattung statt (E5). Wissenschaftliche Evaluationen im Nachgang einer Krise sind bei den Behörden nicht von Bedeutung. Obwohl sich drei Experten dahingehend äußern, dass man aus Fehlern der vergangenen Krisen lernen sollte (E4; E5; E9), ist der 
Stellenwert der Krisennachbereitung und Evaluation insgesamt als niedrig zu beurteilen.

Von einem vernachlässigbaren Stellenwert der Evaluation ist auch mit Blick auf die Risikokommunikation auszugehen. So sagt ein Interviewpartner, nicht über die Mittel zu verfügen, Kommunikationsinstrumente zu evaluieren (E8).

\subsubsection{Krisenvorbereitung}

Ein Experte verweist darauf, dass Maßnahmen der Krisenvorbereitung und der behördlichen Vorsorge im Verhältnis zur Eintrittswahrscheinlichkeit eines Risikos stehen müssen. Dabei wird angezweifelt, ob man Vorsorge für Szenarien treffen muss, die ,eine Wahrscheinlichkeit von Nullkomma irgendwas haben. Oder ob die eingesetzten Mittel nicht sinnvoller irgendwo anders ausgegeben werden können oder müssen [...] “ (E3: A42).

Für Krisen bzw. bestimmte Krisenverläufe, die im Vorfeld nicht abzusehen sind, so wie bei der H1N1-Influenza die Debatte um den adjuvantierten Impfstoff (vgl. Kap. 8.2.9.1), kann es aus Sicht eines Interviewpartners keine Vorbereitung geben (E4).

Als Vorbereitung auf mögliche Krisen werden zwei Ansätze genannt: Krisenpläne und Krisenübungen.

\subsubsection{Krisenpläne}

Es liegen nur vereinzelt interne Leitfäden oder Pläne für Krisenkommunikation in den Behörden vor. Beispielsweise existiert ein Skript, in dem Informationen über die Bundesressorts und deren Krisenmechanismen enthalten sind (E7) ${ }^{33}$. In einem Interview wird deutlich, dass ein internes Krisenkommunikationskonzept, das nach Aussage vorliegt, aus Ablaufplänen und Telefonlisten besteht (E8). Eine andere Behörde besitzt fact sheets, die eine Orientierung für das Organisationshandeln bieten (E9).

Zwei der von den Interviewpartnern angesprochenen Krisenpläne basieren nicht auf konkreten Szenarien. Abläufe werden lieber breit dargestellt, da man sonst ein Leitfaden für jedes vorstellbare Szenario entwickeln müsste. Vielmehr sollten Instrumente erstellt werden, die für jedes Szenario funktionieren (E3; E7).

\footnotetext{
${ }^{33}$ Es gab keine Möglichkeit, dieses einzusehen und weiterführend in die Analyse einzubeziehen, da es als Verschlusssache und nur für den Dienstgebraucht eingestuft ist.
} 
Von einem Interviewpartner werden Krisenpläne als sinnvoll erachtet, vor allem hinsichtlich der Abläufe und Zuständigkeiten in einer Krise. Jedoch ist es ein Problem, dass Krisenpläne nicht Teil der täglichen Aufgaben sind und nur selten Zeit bleibt, sich mit ihnen auseinanderzusetzen. In dieser Behörde wird ein Plan für Krisenmanagement zum Zeitpunkt des Interviews überarbeitet (E3).

In insgesamt fünf Interviews werden Bedenken gegenüber dem Nutzen von Krisenplänen geäußert. Diese sind nicht für die Verwendung in der eigentlichen Krise geeignet oder oft $\mathrm{zu}$ theoretisch, so dass sie in der Praxis nur bedingt nützen bzw. bei gewissen medialen Logiken nicht helfen können (E2; E4; E5; E6; E8). Stattdessen sollte man sich als Behörde bereits in Nicht-Krisenzeiten Gedanken über Krisenkommunikationsprozesse machen (E8). Ein Experte führt aus, dass zwar Checklisten zur Krisenkommunikation existieren, doch diese für die tatsächliche Krisenkommunikation keine Rolle spielen. Erfahrungen mit Krisen, das Lernen aus Fallbeispielen und bisher begangene Fehler sind seiner Ansicht nach wesentlich aufschlussreicher, um aus Krisen und für die nächsten Krisen zu lernen (E5).

Ein weiterer Interviewpartner, dessen Behörde nach Selbstauskunft einen internen Krisenplan besitzt, äußert sich ähnlich. Der Einfluss solcher Pläne ist sehr begrenzt, auch weil in der Krise keine Zeit bleibt, Pläne zu studieren. Wichtiger ist es, dass die agierenden Personen Erfahrungen haben, sich gut verstehen und gemeinsam an einer Lösung des Problems interessiert sind (E4).

Es wird darauf verwiesen, dass der Nationale Pandemieplan mit dem Stand von 2007 zum Zeitpunkt der Interviews (im Jahr 2013) nicht mehr aktuell ist und dringend aktualisiert werden muss (E2; E9). Laut einem Interviewpartner werden die Pandemiepläne regelmäßig angepasst, weil man aus Krisen lernt. Dieses Lernen soll in die kommenden Planungen integriert werden, damit Wissen und Erfahrungen erhalten bleiben, auch für nachfolgende Mitarbeiter (E4). Wie dargelegt wurden seit Dezember 2014 überarbeitete Teile des Pandemieplans veröffentlicht, im September 2015 auch ein Teil zur Kommunikation (vgl. Kap. 8.1.8).

\subsubsection{Krisenübungen}

Insgesamt wird deutlich, dass die Frage nach Krisenübungen meist mit verschiedenen Aussagen zur LÜKEX beantwortet wird. Andere Fortbildungsmaßnahmen sind nicht fest etabliert. Von vier Interviewpartnern wird ausgeführt, dass Mitarbeiter Fortbildungen, z. B. an der AKNZ oder an der Bundesakademie für öffentliche Verwaltung, in Anspruch nehmen können, auch über Themen der Krisenkommunikation (E3; E5; E6; E7). Doch wird betont, dass diese nicht obligatorisch sind (E5; E6). In einer Behörde sollen neu eingestellte Mitarbeiter 
an einer Fortbildung über Krisenmanagementprozesse der Behörde teilnehmen (E7). Weiterbildungen erfolgen in einer weiteren Behörde für Mitarbeiter der Kommunikationsabteilungen bedarfsorientiert, jedoch ohne Fokus auf eine Krise, weil man der Meinung ist, dass die erlernten Kenntnisse auf Krisen übertragbar sind (E9).

An bisherigen LÜKEX-Übungen haben bereits alle befragten Behörden teilgenommen, obgleich nicht immer die jeweiligen interviewten Experten selbst (über eine Behörde kann diesbezüglich keine Aussage getroffen werden, weil die LÜKEX im Interview nicht thematisiert wurde). Es wird herausgestellt, dass die LÜKEX ein zweijähriger Prozess mit Workshops und Fortbildungen ist, in dem man sich abstimmt und Strategien entwickelt. Dieser Prozess wird als Kernstück betrachtet, das dann in den zwei Übungstagen gipfelt. Die Abstimmungen untereinander werden als positiv beurteilt (E7).

Sechs Interviewpartner schreiben den LÜKEX-Übungen einen hohen Nutzen zu. Bei zwei ist dies naturgemäß, da sie für die Entwicklung und Umsetzung der Übung verantwortlich sind. Es wird ausgeführt, dass die reale Übungssimulation über zwei Tage gut schult und aufzeigt, was eine solche Situation für eine Pressestelle bedeutet, z. B. in Bezug auf personelle Kapazitäten. Auch wenn die Teilnahme an der LÜKEX Aufwand und Zeit nach sich zieht, ist es aus Sicht eines Experten für Mitarbeiter einer Pressestelle ein sinnvolles Training (E7). Nach ihm hat sich die Einbindung der Presse- und Öffentlichkeitsarbeit in den Übungen wesentlich verbessert. Vor zehn Jahren wurde dieser Aspekt kaum berücksichtigt, mittlerweile sind die Übungen weiter ausdifferenziert, so dass man als Kommunikationsverantwortlicher involviert und gefordert ist (E7). Auch in Bezug auf die Vernetzung und Zusammenarbeit mit den beteiligten Behörden und das dadurch entstehende Vertrauen wird der LÜKEX ein hoher Nutzen zugeschrieben (E4; E5; E6).

Die LÜKEX wird insbesondere für Krisen, die selten auftreten oder in dieser Form noch nie aufgetreten sind, als sinnvoll erachtet (E3). Aus der Krisenübung sollten im Anschluss Lehren und Konsequenzen gezogen werden (E3). So wird auch ausgeführt, dass die LÜKEX 2007, der ein Influenzapandemieszenario zugrunde lag, als ein Ausgangspunkt dafür gilt, dass es nun Überlegungen zur Reformierung der ENV gibt (E6).

Zudem wird die LÜKEX als Möglichkeit für die Risikokommunikation gesehen:

„Ich glaube, dass die LÜKEX ein sehr gutes Mittel zur Sensibilisierung ist. Für die Risikokommunikation, wenn Sie so wollen, ein exzellentes Tool." (E8: A82) 
Einige Interviewpartner sehen in der LÜKEX zwar ebenfalls einen Nutzen, doch weisen auch auf grundsätzliche Mängel der Übung hin (E2; E6; E8). Demnach wird keine adäquate Kritik geübt und die eigentlichen Herausforderungen der Übung werden nicht thematisiert. Als Grund dafür wird genannt, dass es unbequem ist, sich mit den identifizierten Problemen auseinanderzusetzen (E2; E6). Entsprechend werden auch die Auswertungsberichte sehr kritisch betrachtet. Diese sind nur für eingeschränkte Kreise einsehbar, euphemistisch gehalten und sollen „möglichst keinem wehtun“ (E2: A56) (E2; E6; E8). Es wird gefordert, dass der Umgang mit der LÜKEX offener und transparenter wird. Derzeit geschieht das bewusst nicht, um niemanden zu verprellen. Wenn Behörden Missstände und Fehler offener aufzeigen würden, gingen sie das Risiko ein, dass sich Übungsteilnehmer wie Bundesländer, Unternehmen oder Verbände nicht mehr beteiligen (E8).

\subsubsection{Interorganisationale Risiko- und Krisenkommunikation}

Bei den Fragen nach interorganisationaler Risiko- und Krisenkommunikation dominierten Aussagen über Zuständigkeiten. Dies wird nachfolgend dargelegt. Zudem werden die Ergebnisse zur OVP aufgeführt, die auch die unterschiedlichen Verständnisse der OVP umfassen.

\subsubsection{Zuständigkeiten, Zusammenarbeit zwischen Behörden}

Neben den Behörden, die befragt wurden, werden vor allem Behörden auf Bundesländerebene genannt, mit denen man sich in Krisen abstimmen müsste. Alle Behörden verweisen auf die Zuständigkeit der Bundesländer in Krisen, sofern es sich um Nicht-Verteidigungsfälle handelt. Von einem Interviewpartner heißt es, dass das Verhältnis zu den Bundesländern oft ambivalent ist, da diese einerseits auf ihre Unabhängigkeit bestehen und nicht möchten, dass der Bund ihnen Vorschriften macht. Andererseits wenden sie sich in Krisen an den Bund, wenn sie Unterstützung bei der Kommunikation benötigen (E7).

In fast allen Interviews erfolgen Erläuterungen über Zuständigkeiten, obgleich dies nicht durch Fragen thematisiert wurde. So weisen mehrere Interviewpartner darauf hin, dass sie nicht oder nicht ausschließlich für Krisenkommunikation in einem bestimmten thematischen Bereich zuständig sind respektive erfolgen detaillierte Ausführungen darüber, warum sie für bestimmte Aufgaben, die sich nicht immer auf Kommunikation beziehen, nicht zuständig sind. Es liegen mehrere unstimmige Aussagen hinsichtlich der Zuständigkeiten für Krisenkommunikation vor, damit gehen Zurückweisungen von Zuständigkeiten einher, die 
der entsprechenden Behörde jedoch von anderen Interviewpartnern bescheinigt wurden.

Sämtliche Ausführungen über Zuständigkeiten implizieren schwierige, teils konfliktträchtige, zwischenbehördliche Verhältnisse, sowohl zwischen Behörden auf Bundesebene als auch zwischen Behörden auf Bundes- und Landesebene. Auch hinsichtlich abzustimmender Kommunikationsprozesse werden Spannungen deutlich.

Für eine funktionierende Zusammenarbeit zwischen den Behörden werden schnelle Absprachen, kurze Wege, Kooperationen sowie die Einigung auf einen Konsens als Erwartungen formuliert (E6; E9). Zudem werden Transparenz und eine einheitliche Informationsbasis aller beteiligten Behörden erwartet, jeder sollte wissen, wer wann was sagt (E3; E7). Wenn in einer Krise mehrere Behörden beteiligt sind, sollten diese bereits im Vorfeld miteinander kommuniziert haben, um sich besser einigen zu können. Diese gegenseitige Informierung wird als essentiell erachtet, jedoch unter Berücksichtigung der Zuständigkeiten (E2).

Es ist erkennbar, dass keine strategische Ausrichtung einer zwischenbehördlichen Risiko- und Krisenkommunikation vorliegt. Dies belegen auch bisherige Krisen wie die H1N1-Influenza und die EHEC-Epidemie (vgl. Kap. 8.2.9).

\subsubsection{One-voice-policy}

Das Sprechen mit einer Stimme wird von fast allen Interviewpartnern grundsätzlich als wichtig, teilweise gar als „Idealfall“ (E7: A101) erachtet. Dennoch wird die OVP hinsichtlich der Umsetzung auch mehrfach kritisch gesehen:

,[...] aus der praktischen Sicht mache ich mir keine Illusionen, im Falle einer Krise wird man nie alles so steuern können, dass es nur die eine Information aus einem Guss gibt. “ (E7: A101)

Sobald mehrere Ressorts betroffen sowie Bund und Länder involviert sind, ist eine Umsetzung nicht praktikabel (E2; E5; E6). Aus Sicht eines Experten muss ein Landes- oder Bundesminister schnell agieren und hat keine Zeit, sich zunächst mit allen weiteren Beteiligten wegen der Sprachregelung abzustimmen, selbst wenn sich theoretisch alle einig sind. Das funktioniert schon auf Bundesebene nicht, wenn Bundesländer involviert sind erst recht nicht. Eine Abstimmung würde aufgrund der vielen Akteure und Hierarchieebenen zu lange dauern (E2). 
, [...] wir werden die eine Stimme im Föderalismus nicht haben. Wir werden immer eine Botschaft haben, die, wenn es gut läuft, durch viele Minister, die alle laut Grundgesetz ihren Pflichten nachkommen, gleichermaßen kommuniziert wird. Aber wichtig ist, dass die vielen Gesichter und die vielen Stimmen immer mit einer Sprache sprechen." (E5: A88).

Dieses Zitat zeigt, dass auch eine gemeinsame Sprache als eine Form der OVP erachtet wird. Ein weiteres Verständnis der OVP betrifft die Einrichtung einer zentralen Stelle, die als einzige befugt ist, Informationen zu veröffentlichen. Zugleich wird eine solche als praxisfern erachtet, insbesondere, weil diese nicht alle fachlichen Facetten abdecken könnte (E2; E7). Auch Meinungsvielfalt, die zu einer demokratischen Gesellschaft dazu gehört, stellt einen Grund für eine erschwerte Umsetzung der OVP dar (E8). Ein Experte gibt zu bedenken, dass die OVP nur begrenzt funktionieren kann, weil es immer Personen gibt, die Kritik äußern oder anderer Meinung sind und die von den Medien gezielt gesucht werden (E9).

Ein Interviewpartner differenziert zwischen interner und zwischenbehördlicher OVP. Auf die eigene Behörde bezogen, versteht er die abgestimmten Sprachregelungen als Selbstverständlichkeit. Von einer Behörde sollten nicht verschiedene Meinungen kommuniziert werden (E2).

Ein Interviewpartner zeigt eine durchgehend ablehnende Haltung gegenüber der OVP. Es wird dargelegt, dass es Studien zufolge nicht der Erwartung der Bevölkerung entspricht, ,wenn alle beteiligten Behörden, Institute und Ministerien quasi diktatorisch eine Meinung vertreten würden und aus einem Sprachrohr, womöglich noch in einem Wortlaut, sprechen würden " (E1: A116). Es wird die Ansicht vertreten, dass man auch als Behörde einer veröffentlichten Position widersprechen kann, weil es in Deutschland ein pluralistisches System gibt, das Meinungsfreiheit und -vielfalt zulässt. Wenn es mehrere Stimmen gibt, würde das die Akzeptanz der Aussagen erhöhen. Allerdings räumt dieser Interviewpartner ebenfalls ein, dass es schwer vermittelbar sein kann, wenn Behörden, die auf einer Ebene agieren, konträre Aussagen treffen. Dennoch plädiert er dafür, die Meinungsvielfalt beizubehalten, damit keine Zensur von Meinungen stattfindet (E1). Damit wird die persönliche Meinung des Interviewpartners zum Ausdruck gebracht, nicht die der Behörde.

Die Ausführungen veranschaulichen, dass unterschiedliche Deutungsmuster von OVP hinsichtlich der in der Theorie aufgezeigten Verständnisse (formaler, inhaltlicher, instrumenteller und sprachlicher Art) sowie hinsichtlich der Ebene der Durchführung (intraorganisational, interorganisational) existieren (vgl. Kap. 4.3). 


\subsubsection{Interaktionspartner Medien}

\subsubsection{Pressearbeit über Risiken und Krisen}

Es wird erläutert, wie schwierig es ist, Risikokommunikationsbotschaften medial zu verankern, da Medien anlassbezogen berichten. So würden Medien auch nur dann über ENV berichten, wenn es tatsächlich akut ist und nicht bereits im Vorfeld (E6; E8).

Es wird deutlich, dass es in den meisten Behörden keine explizite Strategie für den Umgang mit Journalisten in der Krise gibt (E5; E6; E7; E8; E9). Das wird auch damit begründet, dass keine Krise wie die andere ist. Man bedient sich eher an bestehenden Instrumenten und wählt je nach Bedarf die entsprechenden Mittel aus (E5). Ein Experte führt aus, dass sich der Umgang mit Journalisten in einer Krise nicht von der Alltagsarbeit unterscheidet. Auf die Frage, welche Maßnahmen der Pressearbeit in einer Krise unbedingt notwendig sind, erläutert er daraufhin auch die klassischen Instrumente der alltäglichen Pressearbeit (E7). Eine Behörde hat Textbausteine und eine Darksite im Internet für bestimmte Krisenszenarien vorbereitet (E8). Ein weiterer Interviewpartner verweist bei Notwendigkeiten, die in einer Krise im Umgang mit Journalisten unbedingt beachtet werden müssen, auf die OVP (E9).

\subsubsection{Verhältnis Behörden und Medien}

Es lässt sich festhalten, dass alle Behörden einen Presseverteiler besitzen und pflegen. Das gehört zur Alltagsarbeit der Pressestellen und wird als wesentlich erachtet. Drei Interviewpartner verweisen auf ein wechselseitiges Verhältnis mit Journalisten, bei dem Anfragen von Journalisten beantwortet werden, aber auch Journalisten in der Pressestelle anrufen und sich nach Neuigkeiten erkundigen. $\mathrm{Zu}$ bestimmten Journalisten werden besonders intensive Beziehungen unterhalten (E1; E5; E9). Im Rahmen dieses festen Netzwerkes herrscht ein vertrautes Verhältnis zwischen Mitarbeitern der Pressestelle und Journalisten (E5), in Hinblick auf Krisen sollte dieses bereits in Nicht-Krisenzeiten hergestellt sein (E9). Ein Interviewpartner führt aus, dass für die Kontaktpflege der Besuch von Veranstaltungen wichtig ist, um sich auch persönlich zu treffen (E6).

Zugleich lassen sich in einigen Interviews kritische Perspektiven auf Journalisten und ihre Arbeitsweisen identifizieren. So wird erläutert, dass Journalisten grundsätzlich neugierig sind und gern spekulieren (E4; E7). Ferner wird dargelegt, dass ungewöhnliche Meinungen sowie von den verantwortlichen Behörden abweichende Meinungen gern für die Krisenberichterstattung verwendet werden. 
Es wird eingeräumt, dass ein strategischer Umgang der Behörden mit Medien diese Schwierigkeit kaum eindämmen kann, weil Medien ihre eigene Logik verfolgen und das Einholen von verschiedenen Meinungen dazu gehört (E2). Ein weiterer Interviewpartner führt analog aus, dass es immer besondere Journalisten mit großem „,Konfliktpotential“ (E4: A138) gibt. Damit sind Journalisten gemeint, die oft das Gegenteil behaupten, um den Behörden die Arbeit zu erschweren (E4). Es wird darauf verwiesen, dass sich Journalisten in Krisen entsprechend ihrer Verantwortung verhalten sollten und wenn z. B. keine Informationen aus validen Quellen vorliegen, lieber auf die Berichterstattung verzichten sollten (E2; E4).

Zudem wird erneut die Schnelligkeit der medialen Berichterstattung thematisiert:

„Ich glaube, wofür die Medien weniger Verständnis haben, ist, wie lange es manchmal dauert, bis eine Behörde dann wirklich mal bestimmte Dinge nach außen veröffentlichen kann. " (E8: A79)

\subsubsection{Erwartungen der Journalisten (Fremdeinschätzung der Behörden)}

Auf die Frage, was die Experten denken, was Journalisten von der Risiko- und Krisenkommunikation der Behörden erwarten, folgen verschiedene Antworten, die sich hauptsächlich auf Krisen beziehen. Auf die Pressearbeit allgemein bezogen erwartet ein Journalist nach Ansicht eines Experten eine Neuigkeit, relevante Informationen für die Zielgruppen des Mediums und dass der Journalist nur die Informationen erhält, die für ihn interessant sind und keine Meldungen darüber hinaus. Dabei wird die Meinung vertreten, dass sich diese Erwartungen in einer Krise nicht unterscheiden (E6).

Die von den Interviewpartnern vermuteten journalistischen Erwartungen an Krisenkommunikation können in Erwartungen inhaltlicher Art sowie Erwartungen an die formale Zusammenarbeit kategorisiert werden:

Inhaltliche Erwartungen:

- $\quad$ Sachliche, korrekte, gesicherte Informationen (6x)

- Schnelle Reaktionen und Informationen (5x)

- $\quad$ Aktuelle Informationen/Behörde soll immer aktuell informiert sein (3x)

- $\quad$ Mit vorherigem einhergehend: aktueller Internetauftritt (2x)

- Transparenz, Offenheit $(2 \mathrm{x})$

- $\quad$ Bewertungen und Einschätzungen der Situation (2x)

- Klärung des Problems (1x)

- Vermittlung des Gefühls von Sicherheit und Gewissheit (1x) 
Formale Erwartungen:

- $\quad$ Eine zentrale Informationsstelle $(2 \mathrm{x})$

- Informationen auch außerhalb der gängigen Geschäftszeiten $(2 \mathrm{x})$

- $\quad$ Erreichbarkeit (2x)

- Möglichkeit für O-Töne und Videoaufnahmen (2x)

- $\quad$ Möglichkeit, Fragen zu stellen (1x)

Gleichermaßen wird darauf verwiesen, dass man als Behörde in einer Krise nicht alle Erwartungen erfüllen kann:

„Sie werden in der Krise nie alle zufriedenstellen können. Man kann eigentlich nur probieren, denen so weit wie möglich die Arbeit zu ermöglichen. "(E2: A60)

„Der [Journalist] erwartet sicherlich eine Menge. Ich möchte mich mal darauf beschränken, was er erwarten darf. Was er von uns erwarten darf, ist eine schnelle und sachbezogene Information über die Fakten, über die Tatsachen." (E7: A102)

\subsubsection{Ernährungsnotfallvorsorge}

Zunächst werden die Aussagen zu real stattfindenden Prozessen der ENV dargelegt. Anschließend werden die Inhalte, die im Rahmen des abgefragten Krisenszenarios über die ENV erfolgten, wiedergegeben.

Es wird darauf hingewiesen, dass Lebensmittelengpässe aufgrund verschiedener Szenarien auftreten können, u. a. Pandemien, Hochwasser oder Stromausfälle (E3).

Die ENV steht vor diesen generellen Herausforderungen:

- $\quad$ Fehlendes Bewusstsein dafür, dass man sich mit Lebensmitteln bevorraten muss, insbesondere, wenn Lebensmittel permanent verfügbar sind

- Fehlende Lagermöglichkeiten in Haushalten, insbesondere in Ballungsgebieten

- $\quad$ Kulturelle Unterschiede in der Gesellschaft (E3; E8) 
Als Instrument der zwischenbehördlichen Risikokommunikation kann das Informationssystem Ernährungsnotfallvorsorge (IS ENV) identifiziert werden. Diese digitale Kommunikationsplattform stellt Daten über die Ernährungswirtschaft für Bundes- und Landesbehörden sowie Kommunen zur Verfügung, im Alltag und in Krisen. Die Plattform wird inzwischen jedoch nicht mehr genutzt, was durch Erkenntnisse aus dem Forschungsprojekt NeuENV bekannt wurde (Stand: November 2015). Darüber hinaus finden zwischen den Bundesbehörden und den Bundesländern circa ein bis zwei Mal im Jahr Treffen zum Austausch über die Ernährungsvorsorge statt.

Es lassen sich drei externe Risikokommunikationsinstrumente über die ENV identifizieren. Neben der Website www.ernaehrungsvorsorge.de beinhalten die Broschüre „Ratgeber für Notfallvorsorge und richtiges Handeln in Notsituationen - Katastrophenalarm“ des BBK (2017; ursprgl. „Für den Notfall vorgesorgt", BBK 2009) sowie die Website der BLE Informationen über die ENV und Ratschläge zur Bevorratung mit Lebensmitteln ${ }^{34}$.

Die externe Kommunikation über die ENV wird von den Behörden bewusst passiv gehalten. Anfragen dazu werden zwar bearbeitet, eine proaktive Ansprache der Journalisten wird hingegen nicht verfolgt. Folgende Gründe werden dafür genannt:

- Prüfung des derzeitigen Konzepts der ENV und mögliche zukünftige Umstrukturierung

- $\quad$ Kritische Betrachtung der derzeitigen Organisation der ENV (in diesem Zusammenhang wird ein Gutachten des Bundesrechnungshofes erwähnt, das die ENV in ihrer jetzigen Form kritisiert, vgl. Bundesrechnungshof 2012)

- Uneinigkeit über die zukünftige Ausrichtung der ENV

- Gratwanderung, ENV zu kommunizieren ohne Panik auszulösen

- Schwierigkeit der Kommunikation, wenn solche Ausnahmezustände in Deutschland nicht vorkommen

- $\quad$ Geheimhaltung der Lagerstandorte - zu viel Kommunikation wäre „kontraproduktiv“

\footnotetext{
${ }^{34}$ Auch die Bundesländer unterhalten mehrheitlich eine Website, auf der sie über ENV informieren oder auf die drei genannten Kommunikationsinstrumente des Bundes verweisen (Braun 2013). Vereinzelt nutzen einige Bundesländer weitere Kommunikationsmaßnahmen über ENV, z. B. das Bundesland Sachsen mit einem Werbespot (vgl. FreistaatSachsen 2013). Dies stellt in der Gesamtbetrachtung jedoch eine Ausnahme dar.
} 
- Kampagne „Zu gut für die Tonne“ widerspricht einer Kommunikation zur Bevorratung mit Lebensmitteln (Kernbotschaft der Kampagne: zu viele Lebensmittel werden weggeworfen, das sollte geändert werden) (E5; E6)

\subsubsection{Berichterstattung über ENV}

Nach Aussage eines Interviewpartners besteht ein reges Interesse bei Journalisten zu erfahren, wo sich die Lebensmittelvorratslager befinden. Dieses wird durch entsprechende Pressefahrten in die Lager gesteigert. Es gab bislang circa ein bis zwei Mal im Jahr eine Fahrt mit Journalisten zu einem der Vorratslager. Diese Pressefahrten und die dazugehörige Berichterstattung werden von dem Experten kritisch beurteilt, da sie Vorwürfe in der Medienberichterstattung wie die folgenden nach sich ziehen: das veraltete Konzept der ENV, die Verschwendung von Steuergeldern, der Verlust von Lebensmitteln und die Beeinflussung der Weltmarktpreise für Lebensmittel (E5).

Es wird die Meinung geäußert, dass die Berichterstattung über ENV zu häufig die Lagerung von Lebensmitteln in den Fokus stellt und andere Aspekte, wie etwa Versorgungsbilanzen, vernachlässigt. Die ENV wird deshalb medial meist nicht ganzheitlich dargestellt. Gleichermaßen wird Verständnis dafür gezeigt, dass sich die Lebensmittellager journalistisch besser aufbereiten lassen als beispielsweise gesetzliche Regelungen (E6).

\subsubsection{Szenario Influenzapandemie mit Lebensmittelengpass}

In vier Interviews konnte das Szenario vorgelesen und die Fragen dazu gestellt werden (E3; E4, E6; E7). In den anderen Interviews gab es durch die vorherigen Ausführungen über Zuständigkeiten oder den Mangel an Zeit keine Gelegenheit, das Szenario zu thematisieren.

\section{Vorbereitung auf das Szenario}

Auf die Frage, inwiefern die Behörden auf das angenommene Szenario hinsichtlich der Kommunikation vorbereitet sind, wird auf Krisenübungen und Krisenpläne verwiesen (E4; E7). In diesem Zusammenhang wird ebenfalls die LÜKEX 2007 erwähnt, durch die man auf ein solches Szenario vorbereitet ist und durch die man auch weiß, welche Probleme auftreten könnten (E6).

Eine Behörde sieht sich auf das Szenario deshalb gut vorbereitet, weil es eine ähnliche Situation - jedoch nicht in diesem Schweregrad - bereits mit der H1N1-Influenza erlebt hat. Es wird ausgeführt, dass man daraus gelernt hat und 
die Personen in den zuständigen Behörden noch immer dieselben Positionen innehaben. Gleichzeitig wird zu bedenken gegeben, dass sich eine Krise vor allem dadurch kennzeichnet, dass Dinge passieren, auf die man sich nicht vorbereiten kann, dann muss man spontan reagieren (E4).

\section{Zuständigkeiten und Abläufe in dem Szenario}

Die Fragen zum zugrunde liegenden Szenario lösen erneut Erläuterungen über Zuständigkeiten aus, die teilweise unterschiedlich dargelegt werden. Betont wird die Hoheit der Bundesländer und der Gesundheitsressorts, die Verantwortung für Risiko- und Krisenkommunikation wird bei den Interviewpartnern nicht bzw. nicht ausschließlich im eigenen Haus gesehen. Auch die Meinungen hinsichtlich der Konstituierung eines Krisenstabs divergieren für dieses Szenario.

Es wird dargelegt, dass in einer Influenzapandemie ein regelmäßiger Austausch zwischen dem federführenden Ministerium, den nachgeordneten Behörden und den Bundesländern stattfinden würde, auch auf kommunikativer Ebene. Dies gilt auch europaweit und für die Zusammenarbeit mit der WHO. Außerdem wird herausgestellt, dass die Zuständigkeiten auch von dem thematischen Fokus der Krisenkommunikation abhängen. Wenn es beispielsweise um Schulschließungen aufgrund der Pandemie geht, wären regionale Ämter für die entsprechende Kommunikation verantwortlich (E4).

Drei Interviewpartner führen aus, dass diese Krise zunächst eine gesundheitliche ist und deshalb initial das Krisenmanagement der Behörden im Gesundheitsbereich gefordert wäre (E3; E4; E7). Entsprechend wird von einer Behörde, die nicht im gesundheitlichen Bereich tätig ist, mehrmals betont, nicht zuständig zu sein.

Die verantwortlichen Behörden im Lebensmittelbereich würden erst aktiv werden, wenn es Probleme in der Lebensmittelversorgung gibt. Dann würden die Bundesbehörden mit den Fachreferenten der Bundesländer zusammenarbeiten (E3; E6). In diesem Zusammenhang wird erneut die Bedeutung der One-voicepolicy betont (E6). Man würde ein Lagebild erstellen und Einschätzungen der Länder einholen, wie die Versorgung dort funktioniert und ob es Engpässe gibt. Gleichzeitig würde man auf die Verbände der Lebensmittelwirtschaft zugehen, um so die Unternehmen gebündelt zu erreichen. Wenn die Wirtschaft die Versorgung in mehreren Bundesländern nicht mehr gewährleisten kann, müsste es eine Kabinettsentscheidung zum Ausrufen einer Krise geben und man könnte die Notvorräte der Lebensmittellager verwenden. Hier wäre jedoch fraglich, inwiefern dafür vorgesehenes Personal, etwa in Großküchen, bei der hohen Krankheitsrate eingebunden werden kann. Außerdem wäre die Verfügbarkeit der 
Nahrungsmittel vermutlich weniger schwierig als die eigentliche Verarbeitung von Getreide zu Mehl und Brot. Bei der LÜKEX-Übung 2007 hatte es Überlegungen gegeben, in einer solchen Situation über Verbände Absprachen mit den Lebensmitteleinzelhandelsunternehmen $\mathrm{zu}$ treffen, so dass beispielsweise ein Supermarkt eine bestimmte Region versorgt, ein anderer Supermarkt eine andere Region usw. Das wäre aber nur nach Absprache und auf freiwilliger Basis möglich (E3; E6).

Es wird deutlich, dass keine Kommunikationsstrategie für das zugrunde liegende Szenario vor liegt, weder auf behördeninterner, zwischenbehördlicher noch auf externer Ebene gegenüber Medien.

\section{Wahrscheinlichkeit des Szenarios}

Ein Experte glaubt, dass das angenommene Influenzapandemieszenario - egal, wie schwer es ausfallen würde - keinen Lebensmittelengpass hervorrufen würde. Dies wurde bereits bei den Pandemien des vergangenen Jahrhunderts (1957, 1968) deutlich. Selbst wenn eine Pandemie erheblich schwerer verlaufen würde als eine schwere Grippewelle, gäbe es seiner Meinung nach keine Probleme der Lebensmittelversorgung. Unter Umständen könnten bestimmte Lebensmittel nicht geliefert werden, aber ein Versorgungsengpass wird für unwahrscheinlich gehalten. Der Experte hat folglich Zweifel, ob das Szenario eine geeignete Referenz für einen möglichen Lebensmittelengpass sein kann (E2).

\subsubsection{Bisherige Krisen}

In den Interviews werden mehrere Fallbeispiele der Krisenkommunikation aus verschiedenen Bereichen herangezogen. Dazu zählen u. a. Extremwetterlagen, die Ereignisse in Fukushima, BSE, SARS, H1N1-Influenza und Beispiele von mit Viren, Bakterien oder Dioxin kontaminierten Lebensmitteln. Nachfolgend werden die Aussagen zu der H1N1-Influenza sowie der EHEC-Epidemie aufgeführt, da diese am häufigsten als Referenz herangezogen wurden und aufgrund des Szenarios der vorliegenden Arbeit die umfangreichsten Einblicke in Hinblick auf Abläufe, Herausforderungen und Beurteilungen der Krisenkommunikation gewähren. 


\subsubsection{H1N1-Influenza}

\section{Herausforderungen der Krise}

Dass der Bund für die Bevölkerung einen Impfstoff mit einem adjuvantierten Wirkstoff vorsah, für die eigenen Mitarbeiter jedoch einen anderen Impfstoff bevorratete, wird von drei Experten thematisiert und von allen drei als kommunikative Herausforderung der H1N1-Influenza beschrieben (E2; E4; E9). Die Beurteilung darüber erfolgt in unterschiedlichem Maß. So spricht ein Experte in diesem Zusammenhang von einer kleinen Krise in der Krise (E4), ein anderer von einem Problem, das bereits zuvor bekannt war, das man vorher aber nicht hätte kommunizieren können. Letzterer sieht die Ursache dieses Problems darin, dass sich zu viele behördliche Stellen mit dieser Thematik beschäftigten (E2). Aus Sicht des dritten Experten resultierte aus dieser Thematik eine besondere kommunikative Herausforderung, da durch die Diskussion um den adjuvantierten Impfstoff die Botschaft, dass mit den Viren ein gesundheitliches Risiko existiert, das unter Kontrolle gebracht werden muss, erschwert wurde. So ging es in der öffentlichen Diskussion vermehrt um Verwaltungsfragen und weniger um die Notwendigkeit einer Impfung. Auch die alarmierenden Szenarien, die öffentlich gezeichnet wurden, sowie die Versuche, dass die Impfmaßnahmen als vorbeugende Schutzmaßnahmen und nicht als Verschwendung von Steuermitteln wahrgenommen werden, stellten kommunikative Herausforderungen in dieser Krise dar (E9).

\section{Bewertung der Krisenkommunikation}

Die Beurteilungen des kommunikativen Umgangs mit der H1N1-Influenza erfolgen von nur drei Experten höchst unterschiedlich und reichen von gut, über gut mit Einschränkungen bis hin zum Aufzeigen diverser Probleme, die während dieser Krise aufgetreten sind.

Eine Behörde beurteilt ihre Krisenvorbereitung und die kommunikativen Handlungen während der H1N1-Influenza als gut. Dabei beruft man sich u. a. auf eine Kampagne, die allerdings schon im Vorfeld der Influenza entwickelt worden war. Die Behörde bewertet insbesondere den kommunikativen Austausch mit anderen Behörden, mit den Bundesländern und den Ärztevertretern als gelungen. Die Kommunikation und die Meldewege werden als inzwischen verbessert beschrieben, hier bezieht man sich u. a. auf eine neue Verwaltungsvorschrift der Bundesregierung (2013, vgl. Kap. 8.1.6.3) (E4). 
Die Behörde hat laut Selbstauskunft aus der Krise gelernt. Was das konkret betrifft und was man für die Zukunft für Lehren daraus zieht, wird auf Nachfrage nicht detaillierter ausgeführt, es heißt nur:

„Ich kann jetzt nicht im Einzelnen sagen, was, aber alles, was gut gelaufen ist, kann man ja wieder aufnehmen und da, wo es Mängel gab, kann man verbessern. Das ist ja immer das Ziel. “(E4: A145).

Ein anderer Experte erläutert hingegen, dass die Botschaft, sich gegen das Influenzavirus impfen zu lassen, die Bevölkerung nachweislich nicht erreicht hat. Er ist der Ansicht, dass die H1N1-Influenza Lehren mit sich brachte und Aspekte aufgezeigt hat, die es zu überdenken gilt. Das abschließende Fazit fällt dennoch überwiegend positiv aus. Man hat die Influenza gut bewältigt, so dass es zu keiner krisenhaften Entwicklung gekommen ist. In Hinblick auf weitere Ausbruchsgeschehen gilt es jedoch, die Kommunikationsstrukturen zu überprüfen und anzupassen (E9).

Die Bewertung eines dritten Experten in Bezug auf die Zusammenarbeit und zwischenbehördliche Kommunikation während der H1N1-Influenza fällt insgesamt negativ aus. Es werden verschiedene Probleme geschildert, die sich wie folgt zusammenfassen lassen: langsame Entscheidungsprozesse, unklare Zuständigkeiten, fehlende kooperierende Denkweise bei den Behörden und entsprechend unzureichende zwischenbehördliche Absprachen, unterschiedliche Vorstellungen über die zu verwendende Sprache in der externen Kommunikation, unzureichende Aufklärungsarbeit gegenüber der Öffentlichkeit, keine Reaktionen der Behörden auf die Diskussion um den adjuvantierten Impfstoff, unterschiedliche Selbstverständnisse der Akteure, die sich auch an politischen Logiken orientieren (E2).

Auf die Frage nach der Evaluation der kommunikativen Maßnahmen werden interne Workshops sowie eine Publikation angesprochen, jedoch wird nichts Detailliertes dazu ausgeführt (E2; E4). Erkenntnisse aus der Krise wurden laut Aussage eines Experten in keinem Konzept oder Ähnlichem verarbeitet (E2). 


\subsubsection{EHEC-Epidemie}

Die EHEC-Epidemie wird von sieben Interviewpartnern als Krise angesprochen (E1; E2; E3; E4; E5; E6; E9).

\section{Herausforderungen der Krise}

Im Zusammenhang mit der EHEC-Epidemie wird einmal mehr auf Zuständigkeiten verwiesen und darauf, dass für Lebensmittelüberwachung und -warnungen die Bundesländer zuständig sind. Damit ergibt sich für die Kommunikation in so einer Krise eine Vielzahl von Behörden auf Bundes- und Landesebene. Während der EHEC-Epidemie betraf das mindestens 16 Ministerien auf Landesebene, tendenziell mehr, weil es jeweils ein Gesundheits- und ein Landwirtschaftsministerium gibt. Verantwortliche Bundesbehörden waren u. a. das BMEL, das BMG, das BfR und das RKI. Es wird herausgestellt, dass sich Gesundheits- und Lebensmittelbehörden die Zuständigkeiten während der EHEC-Epidemie gegenseitig zugewiesen haben. Einerseits hieß es, es sei ein Infektionsgeschehen, wofür die Gesundheitsbehörden verantwortlich sind. Andererseits wurde die Lage als ein Lebensmittelgeschehen betrachtet. Entsprechend hat sich niemand eindeutig verantwortlich gefühlt (E2; E5).

Als Behörde Unsicherheit und Nicht-Wissen zu vermitteln, war aus Sicht zweier Experten eine kommunikative Herausforderung während des EHEC-Geschehens (E1; E5). Es ist bei lebensmittelbedingten Infektionsgeschehen grundsätzlich schwierig, Viren im Labor nachzuweisen. Die Auffindung und Bewertung des Erregers erzeugte bei EHEC mehrere Tage der Unsicherheit (E5). Damit verbunden war die Schwierigkeit, schnell konkrete Handlungsanweisungen zu geben. Aufgrund des damaligen wissenschaftlichen Kenntnisstandes hatten sich die Behörden dazu entschieden, zunächst vom Verzehr bestimmter Lebensmittel abzuraten, auch wenn die Ursache des Erregers letzten Endes nicht in diesen gefunden wurde. Dafür wurden die Behörden kritisiert. Doch wenn die Behörden sich nicht geäußert hätten und eines dieser Lebensmittel der Ausgangspunkt des Erregers gewesen wäre, hätte man ihnen vorgeworfen, wichtige Informationen vorzuenthalten. Mit dieser wissenschaftlichen Unsicherheit umzugehen, stellt eine besondere Herausforderung dar (E1).

\section{Bewertung der Krisenkommunikation}

Eine Behörde beurteilt ihre kommunikativen Handlungen während der EHECEpidemie als positiv und findet es richtig, dass klare Handlungshinweise für den Verzicht auf Lebensmittel ausgesprochen wurden, weil das den Informationen entsprach, die zu diesem Zeitpunkt vorlagen. Absprachen und einheitliche 
Sprachregelungen waren essentiell, damit keine Verwirrung bei den Verbrauchern entsteht. Das hat nach Ansicht von zwei Experten gut funktioniert (E5; E6).

„Bei EHEC ist es um Leben oder Tod gegangen und da gab es eine hohe Disziplin in der Kommunikation und aus meiner Sicht haben die Behörden hervorragend und sehr schnell gearbeitet, sie konnten den Erreger isolieren. "(E5: A89)

Zugleich wird durch die Interviews deutlich, dass es bei der Zusammenarbeit zwischen verschiedenen Behörden Unstimmigkeiten gab und es wird ausgeführt, dass es bei ähnlichen Geschehnissen zukünftig eine verbesserte Kommunikation geben muss, die bei so vielen Beteiligten vor allem besser gebündelt werden muss. Außerdem soll es in Zukunft eine Task Force geben, die bei lebensmittelbedingten Ausbrüchen ermittelt. Während der EHEC-Epidemie wurde sie spontan initiiert, bei dem Nachweis von Noroviren in Lebensmitteln kam sie erneut zusammen (E5). Ein Experte führt aus, dass die EHEC-Epidemie dazu geführt hat, dass die Meldewege zwischen den Behörden auf Bundes- und Landesebene verkürzt werden und es auch rechtliche Überlegungen gibt, die Zusammenarbeit zwischen Bund, Fachbehörden und Ländern zu optimieren (E4). Damit ist vermutlich die Verwaltungsvorschrift über die Koordinierung des Infektionsschutzes gemeint (vgl. Kap. 8.1.6.3). 


\subsection{Mediale Logik bei Risiken und Krisen: Ergebnisse der Leitfadeninterviews}

\subsubsection{Ziele der Berichterstattung}

In den Interviews mit Journalisten wurde zunächst ermittelt, welche Ziele Journalisten mit ihrer Berichterstattung grundsätzlich verfolgen. Aus diesen Aussagen kann das Selbstverständnis der Journalisten respektive des Ressorts oder des Mediums, für das sie arbeiten, abgeleitet werden.

Die befragten Journalisten geben die nachfolgend zusammengefassten Ziele an:

- Neutral berichten bzw. dazu beitragen, dass sich das Publikum ${ }^{35}$ ein eigenes, möglichst neutrales Bild machen kann $(4 \mathrm{x})$

- Fundierte, zuverlässige Informationen anbieten (3x)

- Serviceorientierte Berichterstattung und Ratschläge für den Alltag anbieten (3x)

- Ausgewogene Berichterstattung anbieten (Pro und Contra eines Themas abbilden) (2x)

- $\quad$ Aktuelle, neue Erkenntnisse aus der Wissenschaft anbieten (2x)

- $\quad$ Perspektive des Publikums berücksichtigen (1x)

- $\quad$ Missstände aufklären $(1 \mathrm{x})$

- Wissenschaft beaufsichtigen, kontrollieren (1x)

- Ausbau eines wissenschaftlichen Verständnisses in der Bevölkerung (1x)

- Verfolgung des staatlichen Auftrages, dazu zählt u. a.: Wirklichkeit abbilden, zur Willensbildung in Deutschland beitragen, Einhalten von ethischen und rechtlichen Rahmenbedingungen (1x, öffentlichrechtliche Medienanstalt)

Von allen Interviewpartnern wird ausgeführt, dass sich die zuvor genannten Ziele der Berichterstattung in einer Krise nicht ändern. Es wird eher entgegengesetzt argumentiert: In der Krise ist es umso wichtiger, gewisse Ziele konsequenter zu verfolgen $(\mathrm{J} 1 ; \mathrm{J} 4)$, etwa das Einholen verschiedener Meinungen von Experten, um damit sämtliche Facetten eines Krisenthemas zu beleuchten:

„Je komplexer das wird, desto mehr Stimmen holen wir eigentlich auch ein. “(J1: A157)

\footnotetext{
${ }^{35}$ Der Terminus Publikum umfasst im Nachfolgenden stets sämtliche Leser (Print und Online), Zuhörer und Zuschauer.
} 
Eine genaue Erklärung wissenschaftlicher Sachverhalte wird ebenfalls als Ziel beschrieben, das in der Krise intensiver verfolgt wird:

„Ich habe eher das Gefühl, dieser Aufklärungsinstinkt verschärft sich immer in so einer Krise, weil dann auch so viele Leute kommen und Unsinn erzählen." (J4: A190)

\subsubsection{Berichterstattung über Risiken und Krisen}

\section{Anlassbezogenheit der Berichterstattung}

In zwei Interviews wird die Anlassbezogenheit der aktuellen Berichterstattung erwähnt (J2; J4). Der Aussage eines Experten, dass es als Behörde sehr schwer ist, Risiken medial zu verankern, da ausschließlich anlassbezogen berichtet wird (E8), wird von einem Journalisten zugestimmt:

„Das ist ja das Nachrichtengeschäft. Wenn es keine Anlässe gibt, sehen wir uns nicht genötigt, irgendwas zu machen oder irgendwelche Risiken zu beschreiben ,Wenn der Fall einträte, dann könnte das und das sein...' Das ist für uns gar keine Nachricht. Das kann man so im Hintergrund speichern [...]. “(J5: A193)

In diesem Zusammenhang wird jedoch auch darauf verwiesen, dass Anlässe geschaffen werden können, ein Instrument, dessen sich z. B. Politiker täglich bedienen. So sind etwa die LÜKEX-Übungen oder große Konferenzen über Sicherheitsthemen durchaus berichtenswert (J2).

Recherche- und Arbeitsweise in der Krise

Bei allen Interviewpartnern besteht Konsens, dass sich die grundsätzlichen Mechanismen der Recherche und der journalistischen Arbeit in einer Krise nicht verändern. So gilt weiterhin das Zwei-Quellen-Prinzip und man versucht viele verschiedene Quellen zu nutzen (J1; J2). Von einem Journalisten wird geäußert, dass es für Krisen eine gewisse journalistische Routine gibt und Vorgaben existieren, nach denen die Recherche verläuft (J5). Als Unterschied wird ausgeführt, dass die Recherche in einer Krise hektischer verläuft (J4).

Egal welche Art der Krise vorliegt, es gilt, dass neben Behörden immer auch die Meinungen von neutralen Experten und Wissenschaftlern in die Berichterstattung einbezogen werden. Dabei wird angemerkt, dass einem durchaus bewusst ist, dass „,der unabhängige Experte“ in dem Sinne nicht existiert (J3). 
Darüber hinaus wird ausgeführt, dass in der Krise Veröffentlichungsdruck herrscht. Um zu veröffentlichen, benötigen Journalisten wiederum Ansprechpartner. Stehen keine Ansprechpartner, etwa von den Behörden, zur Verfügung, werden andere Informationsquellen genutzt, auch wenn diese unter Umständen nicht die gleiche Expertise und Validität aufweisen (J1; J4). Demzufolge dürfen Behörden in einer Krise nicht zu langsam agieren und sollten sich dem journalistischen Rhythmus anpassen (J1).

\section{Herausforderungen der Krisenberichterstattung}

Bei der EHEC-Epidemie bestand eine Herausforderung für einen Journalisten in dem großen Druck, in kurzer Zeit viel verstehen, recherchieren und dann aufklären zu müssen. Insbesondere, weil die Ansprechpartner, auch in den Behörden, alle sehr beschäftigt waren. Gleichzeitig war die EHEC-Epidemie dahingehend journalistisch interessant, dass sich Entwicklungen sehr schnell vollzogen haben, so dass es jeden Tag etwas Neues zu berichten gab. Bei der H1N1-Influenza hingegen, die sich über mehrere Monate erstreckte, ist oft lange Zeit nichts Neues passiert. Dennoch verlangten die Chefredakteure teilweise, Bericht zu erstatten. Dieser Umstand wird als problematisch beurteilt, weil dann nach Themen gesucht wird und nur vermeintliche Diskussionen entstehen. Gleichzeitig soll keine Angst vergrößert werden. Aus journalistischer Perspektive sind solche lang andauernden Krisen demnach wesentlich herausfordernder für die Berichterstattung als plötzliche Krisen, die verhältnismäßig schnell wieder vorüber sind (J4).

Auch im zugrunde liegenden Szenario stellt sich die Frage, ob sich das Thema für die Berichterstattung erschöpft, weil das Szenario einen Zeitraum von sechs bis sieben Monaten umfasst. So äußert ein Journalist, dass die Krisenberichterstattung nachlassen würde, wenn ein gewisser Gewöhnungsprozess eintritt (J2). Ein anderer Interviewpartner betont, dass es vor allem darauf ankäme, welche verschiedenen Aspekte man hervorheben kann. Er geht davon aus, dass es in diesem Szenario immer etwas Neues zu berichten gäbe, da das Szenario sehr extrem ist (J5).

\section{Krisenkommunikation von Behörden}

Inwiefern die behördliche Krisenkommunikation ein Thema für die Krisenberichterstattung darstellt, wird unterschiedlich beurteilt.

Zum einen wird offen geäußert, dass dies ein wichtiges Thema ist und Journalisten gern darüber berichten, wenn Behörden in ihrer Krisenkommunikation Fehler begehen und wenn Missstände aufgezeigt werden können. Nicht zuletzt auch deshalb, weil die behördliche Krisenkommunikation - insbesondere in der Aus- 
einandersetzung mit Bund- und Länderkompetenzen - oft als unzureichend und fehlerhaft beurteilt wird (J1; J5). Demnach wird es als Automatismus betrachtet, dass Journalisten frühzeitig (nach der eigentlichen Information, was geschehen ist) über die Krisenkommunikation der Organisationen berichten, dies steckt in der ,journalistischen DNA" (J1: A156). So wird von drei Interviewpartnern konstatiert, dass es immer Journalisten gibt, die der Frage nachgehen, was falsch läuft und die Krisenmanagement und Krisenkommunikation kritisieren, auch noch im Nachgang einer Krise (J1; J3; J5). Oft rückt die Diskussion darüber sogar während der Krise in den Fokus der Berichterstattung (J1).

„Das ist ja dann ein mindestens so interessanter Aspekt wie die Krise an sich." (J5: A195)

Im Gegensatz dazu erläutern zwei Interviewpartner, dass die Krisenkommunikation der Behörden nur nebensächlich für die Krisenberichterstattung ist (J2; J3). Wenn die Krisenkommunikation und die Handlungen, die damit verbunden sind, ehrlich sind, würde man nicht negativ berichten (J2). Dieser Journalist spricht dabei insbesondere für die öffentlich-rechtlichen Medien und liefert den Einwand, dass man das nicht für andere Medien garantieren kann (J2). Interessanterweise äußert sich ein anderer Interviewpartner der öffentlich-rechtlichen Medien gegenteilig, indem er darlegt, dass man sehr an solchen Themen interessiert ist (J5).

Zwei Mal wird ausgeführt, dass man nur unter besonderen Umständen über die Krisenkommunikation berichten würde, z. B. erst nach einer Krise und auch nur dann, wenn etwas fehlerhaft war (J3) oder erst dann, wenn eine gewisse Frustrationsschwelle überschritten wurde (J4). Kritische Artikel über die behördliche Kommunikation sind eher im Politikressort, wo mehr Kommentare und Meinungsartikel publiziert werden, zu finden. Für Wissenschaftsressorts sind derartige Artikel eine Ausnahme (J4).

Im zugrunde liegenden Influenzapandemieszenario würde man nicht gleich von Beginn an über die behördliche Krisenkommunikation berichten, aber relativ schnell. Wenn es Tote gibt und Kindertageseinrichtungen geschlossen sind, würde man auch die Krisenkommunikation betrachten und prüfen, ob alles richtig gemacht wird und was sich verbessern lässt (J1).

\subsubsection{Verhältnis Journalisten und Behörden in Bezug auf Risiken}

Die befragten Journalisten haben zahlreiche Kontakte zu behördlichen Pressestellen und stehen mit diesen in regelmäßigem Austausch. 
Das persönliche Verhältnis zu den Mitarbeitern der behördlichen Pressestellen wird von allen Journalisten als wichtig herausgestellt, z. B. bei der Informationsweitergabe in einer Krise, die für das Hintergrundwissen des Journalisten eine große Rolle spielt und nicht direkt in die Berichterstattung einfließt. Das funktioniert nur, wenn das Verhältnis zueinander grundsätzlich etabliert und Vertrauen zwischen den verantwortlichen Personen hergestellt ist (J3; J4). Zudem muss auch das grundsätzliche Vertrauen in Behörden als Informationsquellen gegeben sein, so dass z. B. deren Pressemitteilungen automatisch als wichtig und glaubwürdig bewertet werden (J4). Ein Journalist führt aus, dass die Pressemitteilungen von Bundesbehörden einen hohen Stellenwert haben (J1).

Ein Journalist betrachtet das Verhältnis zu den Behörden als gut (J2). Zwei andere konstatieren, dass das Verhältnis von der jeweiligen Behörde und dem jeweiligen Ansprechpartner dort abhängt (J1; J4). Zu einigen Behörden wird ein intensiver, zu anderen ein weniger enger Kontakt gehalten. Das Verhältnis ist u. a. davon abhängig, auf welcher Ebene die Behörde tätig ist. Je kommunaler der Tätigkeitsbereich, desto schwieriger gestaltet sich eine mögliche Zusammenarbeit, so ein Interviewpartner (J1).

Eine Behörde wird in Hinblick auf eine Zusammenarbeit mehrmals als PositivBeispiel genannt (J1; J3; J4). Von einem Journalisten wird die Kommunikation dieser besonders gelobt, weil bislang alle Erwartungen, die der Journalist an die behördliche Kommunikation hatte, erfüllt wurden. Das betrifft vor allem schnelle und zuverlässige Informationen sowie eine übersichtliche Website. Zudem existiert ein sehr gutes persönliches Verhältnis (J3).

Zugleich wird auch Kritik an dem Verhältnis zu Behörden geäußert, etwa in Hinblick darauf, dass Informationen bewusst zurückgehalten werden (J2; J4). So wird ein Fachzentrum einer Behörde kritisiert, da sich dieses während der H1N1Influenza nicht über Experimente zur Übertragbarkeit des Virus äußern wollte. Dafür existiert beim Interviewpartner kein Verständnis, weil er der Meinung ist, dass dieses Zentrum als öffentliche Organisation zur Information verpflichtet ist. Er erläutert, dass sich das Zentrum für dieses Verhalten Monate später entschuldigte und versicherte, beim nächsten Mal Auskunft zu geben (J4). Ferner wird kritisiert, dass Journalisten oft als "Sprachrohr" (J4: A184) von Behörden verstanden werden, bei denen man das Gesagte noch einmal gegenlesen und ggf. korrigieren kann. Diese Ansicht wird vom Interviewpartner mitnichten geteilt (J4). Er betrachtet auch den Begriff „Zusammenarbeit“ zwischen Behörden und Medien als kritisch:

„Das klingt so, als würden wir gemeinsam entscheiden, was wir der Öffentlichkeit... das ist ja nicht so. " (J4: A187) 
Viel eher wird von dem Journalisten ein stärker ausgeprägter Service-Gedanke der Behörden erwartet (vgl. nachfolgende Erwartungen an die Risikokommunikation), nach dem Behörden Journalisten etwas anbieten sollten, ähnlich wie in den USA. Bei den deutschen Behörden hingegen herrscht das Verständnis, dass es die Pflicht der Journalisten ist, behördliche Informationen zu verbreiten. Hier gibt es aus Sicht des Interviewpartners noch Optimierungsbedarf in Bezug auf das Selbstverständnis der Behörden (J4).

\subsubsection{Erwartungen an Risikokommunikation von Behörden}

Die Erwartungen, die an die behördliche Risikokommunikation bestehen, werden nachfolgend kumuliert und in Reihenfolge der Häufigkeit der Nennungen aufgeführt:

- $\quad$ Risiken offen und aktiv thematisieren (2x)

- Ganzheitliche Darstellung eines Risikothemas (Vor- und Nachteile eines Risikos darlegen) (2x)

- $\quad$ Schnell reagieren und informieren $(2 x)$

- Serviceorientierung gegenüber Journalisten/Berücksichtigung der journalistischen Denkweise $(2 \mathrm{x})$

- Feste Ansprechpartner, auch in Bezug auf unterschiedliche Themen (2x), damit einhergehend: klare Struktur der behördlichen Risikokommunikation, die verdeutlicht, wer Ansprechpartner für Journalisten sind $(1 \mathrm{x})$

- Zuverlässige Informationen bereitstellen (1x)

- Transparente Kommunikation (1x)

- Unsicherheiten kommunizieren (1x)

- $\quad$ Für den Laien verständliche Risikokommunikation (1x)

- $\quad$ Anlässe für Risikokommunikation schaffen (1x)

- Behördenmitarbeiter sollen Ansprechpartner bei den Medien kennen und persönliche Beziehungen aufbauen (bereits in NichtKrisenzeiten) (1x)

- Wissenschaftler bzw. Personen mit Fachwissen als Ansprechpartner (nicht ausschließlich Pressesprecher) (1x)

Es wird mehrfach deutlich, dass Journalisten eine Geschichte gegenüber ihrem Publikum zu erzählen haben, wofür sie die richtigen Ansprechpartner benötigen. Die Erwartungen an die behördliche Risikokommunikation verfolgen demnach das übergeordnete Ziel, diese Geschichte erzählen zu können (J4). 
In einem Beispiel wird ausgeführt, dass eine Verwaltungsvorschrift veröffentlicht wurde (Die Bundesregierung 2013, vgl. Kap. 8.1.6.3), in der dazugehörigen Pressemitteilung jedoch nicht stand, was neu oder innovativ daran ist. Das hatte der Interviewpartner jedoch erwartet. Dies wird als intransparente Kommunikation aufgefasst, die einen Journalisten eher motiviert, umfassender zu recherchieren:

„Je mehr man das Gefühl hat, da wird irgendetwas verborgen oder verschleiert, desto mehr versucht man da auch reinzustechen. "(J1: A152)

Von zwei Interviewpartnern wird ausgeführt, dass sie ihre Erwartungen - in Bezug auf schnelle und zuverlässige Informationen sowie in Bezug auf die generelle Ansprechbarkeit der Behörden - als grundsätzlich erfüllt sehen (J2; J3).

\subsubsection{Verhältnis Journalisten und Behörden in Krisen}

Nach Aussage eines Interviewpartners stellt eine Krise eine andere Situation dar als das normale Tagesgeschäft. Die Pressestellen der Behörden stehen dann unter Druck. Dadurch ist die Belastung bei den Behördenmitarbeitern höher und das Verhältnis gestaltet sich anders als im Alltag (J3).

Ein Interviewpartner beschreibt, dass sich Behörden in Krisen eher „gezwungen" fühlen zu kommunizieren. Sobald das Krisenthema wieder von der öffentlichen Agenda verschwindet, kommunizieren sie nicht mehr darüber. Wenn Behörden in einer Krise kommunizieren möchten, würde sich kaum ein Medienvertreter weigern, darüber zu berichten (J5). Behörden werden demnach vor allem als reaktiv und auf öffentlichen Druck hin handelnde Organisationen betrachtet:

\section{„Da sind es ja eher die Medien, die die Behörden vor sich herumtreiben als umgekehrt."(J5: A192)}

Zwei Interviewpartner von öffentlich-rechtlichen Medien führen aus, dass man in der Krise eher kooperativer gegenüber den Behörden agieren würde und grundsätzlich bereit ist, vertrauensvoll zusammenzuarbeiten (J2; J5). Es wird eine Verantwortung bei den öffentlich-rechtlichen Medien gesehen, die den Auftrag der Aufklärung und der Verbreitung von Information haben (J2; J5 $)^{36}$. In extremen Krisen würde man Pressekonferenzen übertragen und Interviewflächen bereitstellen. Auf diese Weise soll die Kommunikation zwischen Staat und

\footnotetext{
${ }^{36}$ Einer dieser beiden Interviewpartner arbeitet für eine öffentlich-rechtliche Medienanstalt, die ein offizieller Teil des Katastrophenschutzsystems in Deutschland ist. Demnach besteht eine gesetzliche Pflicht, Warnmeldungen zu verbreiten.
} 
Bevölkerung durch die (öffentlich-rechtlichen) Medien erleichtert werden (J2). Gleichermaßen wird betont, dass man deswegen kein ,Staatssender" (J2: A164) ist und Informationen unverarbeitet weiterleitet (J2). Man sollte eine kritische Distanz gegenüber Behörden bewahren (J5). Einer dieser beiden Journalisten erläutert, dass man die Berichterstattung einschränken oder aussetzen würde, wenn Menschenleben dadurch geschützt werden können. Dafür müssten die Behörden jedoch überzeugend begründen, dass eine Berichterstattung eine Gefahr darstellt (J2).

Als Belege werden zwei Beispiele näher geschildert, bei denen ausgewählte Medien und Behörden kooperiert haben: die Vereitelung eines terroristischen Anschlages und eine Geiselnahme. In beiden Fällen gab es Absprachen und Vereinbarungen mit Behörden, nichts oder nur bestimmte Informationen zu veröffentlichen. Als Medienorganisation hatte man sich in diesen Fällen selbst beschränkt, weil die Behörden schlüssig darlegen konnten, dass mit einer Berichterstattung das Leben von Menschen in Gefahr gewesen wäre. Der Interviewpartner betont, dass man nur für seine eigene Organisation und nicht für andere Medien sprechen kann. Da eines der Geschehen bereits mehrere Jahre zurückliegt, wird zudem in Frage gestellt, ob solche strikten Vorgaben aufgrund von Social Media heutzutage noch von allen eingehalten werden würden (J2).

Die Ausführungen eines anderen Interviewpartners erfolgen nahezu gegenteilig: Er legt dar, dass man in der Krise versucht, die involvierten Behördenmitarbeiter mit kritischen Meinungen von außenstehenden Experten bewusst zu konfrontieren, um sie unter Druck zu setzen. Das muss nicht geschehen, aber wenn die Krise komplex und undurchsichtig wird, wäre das ein probates Mittel der Konfrontation (J1). Ein weiterer Interviewpartner äußert den für ihn geltenden Grundsatz, dass Wissen immer besser ist als Nicht-Wissen und man aus journalistischer Sicht grundsätzlich keine Informationen zurückhalten würde. Selten sind Ausnahmen denkbar, z. B. im Sicherheits- oder Wirtschaftsbereich (J3).

Ein Interviewpartner vertritt die Meinung, dass sich die Kommunikation der Behörden, aber auch das eigene journalistische Verhalten in der Vergangenheit von Krise zu Krise verbessert hat, da alle dazu lernen. Als Journalist weiß man nun eher, wen man ansprechen kann. Entsprechend zeigt sich der Interviewpartner mit der behördlichen Kommunikation während der EHEC-Epidemie zufriedener als während der H1N1-Influenza, dennoch existiert seiner Meinung nach Verbesserungsbedarf für die behördliche Krisenkommunikation (J1).

Aus Sicht eines Journalisten haben die Behörden während der H1N1-Influenza richtig gehandelt, als sie früh vor dem Virus gewarnt haben. Auch die Entscheidung über die Entwicklung und Produktion des Impfstoffes wird von ihm als 
angemessen empfunden (J3). Die Diskussion um den adjuvantierten Impfstoff wird von einem anderen Interviewpartner hingegen als ,Kommunikations-GAU“ (J1: A159) bewertet, der so nicht passieren darf.

Es wird teilweise Verständnis gegenüber den Behörden für ihre Arbeitsweisen, Strukturen und Beweggründe in der Krise geäußert (J1). Den Journalisten ist bewusst, dass in einer Krise immer eine Vielzahl von Journalisten Ansprechpartner der Behörden benötigen und dass eine Behörde das meist nur bedingt leisten kann (J3). Zudem wird vereinzelt Verständnis dafür gezeigt, wenn Behörden Informationen zurückhalten, um Panik zu verhindern (J2; J5).

\subsubsection{Erwartungen an Krisenkommunikation von Behörden}

Die Erwartungen, die an die behördliche Krisenkommunikation bestehen, leiten sich sowohl aus der Frage nach den Erwartungen an die Krisenkommunikation allgemein als auch aus der Frage nach den Erwartungen in dem Influenzapandemieszenario ab. Sie werden nachfolgend kumuliert aufgeführt:

- $\quad$ Offen und aktiv kommunizieren $(3 x)$

- Schnell informieren (3x)

- $\quad$ Ehrlich sagen, was man weiß und was man nicht weiß (3x)

- $\quad$ Transparente Kommunikation (3x)

- Unsicherheiten kommunizieren (3x)

- Hintergrundinformationen liefern (auch mit Vereinbarung möglich, dass diese nicht veröffentlicht werden) $(2 \mathrm{x})$

- Zuverlässige, fundierte Informationen bereitstellen (1x)

- Kontinuierlich kommunizieren, auch wenn es nichts Neues gibt (1x)

- Zugang zu Informationen herstellen (1x)

- Ansprechpartner zur Verfügung stellen, auch solche, die sich vor der Kamera äußern (1x)

- $\quad$ Gleiche Informationen an alle Medien und gleicher Zugang für alle (1x)

„Eine ganz ehrliche Information darüber ,Das wissen wir, das wissen wir nicht. Wir versuchen die Fakten zu beschaffen, morgen vielleicht mehr.' Also die Ehrlichkeit ist glaube ich das Allerwichtigste dabei. " (J2: A171)

Es wird von vier Interviewpartnern als grundsätzlich wichtig erachtet, dass überhaupt eine behördliche, externe Krisenkommunikation stattfindet. Wenn dies nicht der Fall ist, entstehen Gerüchte, Unruhe und Panik (J2; J3; J4; J5). 
„Also ist das $A$ und $O$ von Krisenkommunikation ansprechbar zu sein. “ (J4: A185)

In diesem Kontext wird angesprochen, dass Behörden oft so vorsichtig mit Äußerungen geworden sind, dass sie im Zweifel lieber nichts mehr sagen. Das ist aus journalistischer Perspektive inakzeptabel (J4). Alle Interviewpartner empfinden es zweifelhaft, wenn Behörden Informationen bewusst zurückhalten, ohne Gründe dafür darzulegen. Dabei wird mehrfach und mit Nachdruck die Erwartung formuliert, dass Behörden auch Unsicherheiten kommunizieren sollten (J2; J3; J4). Das kann mit der Aussage verbunden werden, dass man daran arbeitet, bestimmte Informationen zu erhalten. Behörden sollten in dieser Hinsicht auf keinen Fall etwas vortäuschen (J2; J3). So sollten sie eher kommunizieren, dass sie noch nichts wissen oder an einer Lösung arbeiten, als dass sie sich gar nicht äußern. Diese Erwartung wird auch damit begründet, dass die Öffentlichkeit, nicht allein die Medien, Verständnis dafür haben, dass in Krisen nicht immer sofort alle Informationen verfügbar sind. Die Behörden müssen sich dann aber tatsächlich mit den aufgeworfenen Themen beschäftigen und die angefragten Informationen nachliefern (J2; J3).

Entsprechend werden auf die Frage, was Behörden hinsichtlich ihrer Kommunikation in der Krise vermeiden sollten, folgende Aspekte genannt:

- Informationen vorenthalten $(3 \mathrm{x})$

- $\quad$ Panik stiften (3x)

- $\quad$ Falsche Aussagen tätigen, um ein bestimmtes Ziel zu verfolgen (1x)

- Situation unterschätzen (1x)

- Bevorzugung von bestimmten Medien mit Exklusivinformationen (1x)

Zudem werden verschiedene Aussagen darüber getätigt, was auf formaler Ebene von der behördlichen Krisenkommunikation erwartet wird.

Die Kanäle, über die Journalisten in einer Krise am liebsten von den Behörden informiert werden möchten, sind die „klassischen“ Kanäle E-Mail und Telefon (J1; J3; J4). Die Meinungen, welcher der beiden Kanäle wichtiger ist, differieren (J3; J4). Außerdem ist eine übersichtliche und permanent aktuelle Website wichtig (J2; J3). In diesem Zusammenhang erfolgt eine Empfehlung für Darksites, die Behörden in Krisen verwenden sollten (J2). Ein Journalist wünscht sich, dass die Behörden auch Social-Media-Kanäle für die Kommunikation nutzen (J1). Zwei Interviewpartner betonen, dass man grundsätzlich alle verfügbaren Quellen nutzt, auch Social-Media-Kanäle (J2; J5): „Man nimmt alles mit." (J5: A194). Ein Interviewpartner meint, dass Behörden nur Social Media nutzen sollten, wenn sie Ressourcen und Kompetenzen dafür haben (J4). 
Fernsehen benötigt Bilder, um Bericht erstatten zu können. Deshalb wird von Interviewpartnern dieser Mediengattung die Erwartung an Bild- und Tonmaterial formuliert. Diese gilt generell, aber auch in Krisen. Dabei geht es weniger darum, fertige Bilder von einer Organisation zu erhalten, sondern um den Zugang, eigenständig Bilder aufnehmen zu können (J2; J5). Analog ist für das Radio die Bereitstellung von Ansprechpartnern für O-Töne sehr wichtig (J3). Allerdings berichtet ein Fernsehsender auch über eine Krise, wenn keine Bilder dazu vorliegen. Dann kann man Archivmaterial nutzen oder die Nachrichtensprecher die Nachricht vorlesen lassen. Zudem gibt es zunehmend Aufnahmen von Laien, die über Social-Media-Kanäle schnell zugänglich sind (J2; J5).

\subsubsection{Erwartungen an zwischenbehördliche Krisenkommunikation}

Wenn mehrere Behörden in eine Krise involviert sind, sollten schnelle interne Abstimmungsprozesse stattfinden und es sollte verdeutlicht werden, wer für welches Thema zuständig ist und spricht (J4; J5).

\section{Bündelung von Informationen}

In zwei Interviews wird ein Bezug zur EHEC-Epidemie als ein Negativ-Beispiel der zwischenbehördlichen Krisenkommunikation hergestellt. In dieser Krise war nicht erkennbar, wer für welche Informationen zuständig und damit der richtige Ansprechpartner war. Mit diesen Ausführungen wird der Wunsch nach einer Bündelung von Informationen und einer zentralen Anlaufstelle für Informationen geäußert. So müssten sich Journalisten die Informationen nicht von verschiedenen Organisationen besorgen (J1; J4).

„,Wenn ich einen großen Wunsch an die Krisenkommunikation hätte, dann wäre das wirklich ein Kompetenzzentrum, ein Science Center - wie auch immer man das nennt - aufzubauen, wo die Leute nur dafür da sind, die Informationen zu sammeln. Wenn man weiß, ich brauche jetzt die eine Stelle und die kann mir alle meine Fragen beantworten. " (J1: A155)

Mit einem derartigen Zentrum sollte es eine zentrale Stelle oder Person geben, die wissenschaftliche Einordnungen liefern kann (J4). Gleichzeitig sollte sichergestellt sein, dass es sich dabei um eine seriöse Informationsquelle handelt (J1). Einer der beiden Interviewpartner räumt zugleich ein, dass ihm durchaus bewusst ist, dass die Bündelung vieler verschiedener, dezentral vorliegender Informationen eine wesentliche Aufgabe des Journalismus ist. Sich die Informationen wenigstens gebündelt an einer zentralen Stelle abholen zu können, wird jedoch als sehr nützlich empfunden (J1). Wenn es keine zentrale Anlaufstelle für 
Journalisten geben kann, wird zumindest erwartet, dass man auch auf Landesoder Kommunalebene die gleiche Qualität von Informationen erhält. Das sollte grundsätzlich, etwa durch das zuständige Bundesministerium, geregelt sein (J1). Auch der zweite Interviewpartner räumt ein, dass es illusorisch ist, in Deutschland 16 Bundesländer und ihre Ministerien derartig zu reformieren. Aber ein Krisenzentrum, das etwa von BfR, BMEL, BMBF, RKI betrieben wird, wäre sinnvoll (J4).

Von einem dritten Interviewpartner wird die Empfehlung formuliert, auf behördlicher Seite eine Person in der Krise zu haben, die Vertrauen und Zuversicht ausstrahlt und so der Symbolik der Kommunikation dient (J2). Im Fall eines Krisenkompetenzzentrums wäre eine solche Person ebenfalls, insbesondere für Bewegtbilder, hilfreich (J4).

Es wird auch geäußert, dass es ein „One-Health-Konzept“ geben sollte, nach dem Zuständigkeiten in Bezug auf Gesundheit und Lebensmittel zentral zusammengeführt werden. Die EHEC-Epidemie war ein Beispiel dafür, dass man nicht immer strikt zwischen Gesundheit und Lebensmittel differenzieren kann (J4).

\section{One-voice-policy}

Auf die Frage, wie wichtig die Einhaltung der OVP von mehreren Behörden ist, reagieren die Interviewpartner unterschiedlich. Ein Interviewpartner zeigt sich als strikter Befürworter der OVP und bezeichnet sie als ,das Wichtigste überhaupt" (J2: A168). Ein weiteres Urteil lautet, dass die OVP die journalistische Arbeit sehr erleichtern würde, doch in der Praxis häufig nicht stattfindet, auch, weil die Stimme manchmal komplett stumm ist (J4). Dass überhaupt eine Stimme spricht, die offen kommuniziert, ist eine Erwartung, die in diesem Zusammenhang ein weiteres Mal geäußert wird (J5). Die anderen Reaktionen fallen zwiespältiger aus. So wird der Einwand geäußert, dass Botschaften, die von mehreren Behörden abgestimmt und befürwortet worden sind, nicht das beinhalten, was einzelne Behörden darüber denken. Es würde eher Skepsis bei Journalisten auslösen, wenn sich viele Stimmen auf eine verdichtet haben und sich alle Behörden darüber einig sind (J1):

„Je abgestimmter eine Info ist, desto mehr weiß ich auch, wie schwierig es ist diese Info auf einen Nenner gebracht zu haben. " (J1: A157).

Ein weiterer Interviewpartner ist kein Befürworter der OVP, er erachtet sie als nicht notwendig. Er plädiert dafür, dass Meinungsvielfalt auch in der Berichterstattung dargestellt werden sollte. Er empfindet es als problematisch, wenn jemand nur das kommuniziert, was ihm vorgegeben wurde (J3). Von ihm und 
einem weiteren Journalisten wird Verständnis dafür geäußert, dass mehrere Einschätzungen $\mathrm{zu}$ einer Thematik existieren und dass unterschiedliche Behörden verschiedene Informationen dazu veröffentlichen (J3; J5).

\subsubsection{Ernährungsnotfallvorsorge}

Keiner der fünf Journalisten hat bereits zum Thema ENV recherchiert oder publiziert. Die Journalisten wissen nicht, was die ENV in Deutschland thematisch umfasst und wie sie organisiert ist. Dass die Behörden eine Bevorratung mit Lebensmitteln bis zu zwei Wochen empfehlen, ist den Interviewpartnern entsprechend unbekannt und sie gehen davon aus, dass dies auch auf die Bevölkerung zutrifft.

Dennoch erfolgen einige Einschätzungen in Bezug auf diese Thematik. So werden die Interviewpartner bei der Abfrage des Szenarios zum Teil gebeten, sich vorzustellen, welche Auswirkungen das auf ihre Arbeit in Hinblick auf die Lebensmittelversorgung hätte. Dadurch, dass es so eine Krise in Deutschland bislang noch nicht gegeben hat, wird geäußert, dass es schwer fällt, sich so etwas vorzustellen (J1). Bereits eine Krise, die einen Lebensmittelengpass auslöst, wird als kaum vorstellbar eingeschätzt (J5). Ein Interviewpartner äußert sich besonders kritisch über das ENV-Konzept:

„Lebensmittel zu lagern, das ist anachronistisch. Das stammt ja aus dem 19. Jahrhundert. [...] Daran sieht man aber auch, dass da Behörden zugange sind, die immer noch völlig dilettantische Vorstellungen darüber haben, was passiert, wenn es mal wirklich knallt. "(J5: A191)

Ein Journalist glaubt, dass für die Beschaffung von Lebensmitteln in einer Krise Social Media für die Bevölkerung eine sehr große Rolle spielen würden. Man würde über Facebook, Twitter und weitere Online-Kanäle versuchen Informationen darüber zu bekommen, wo Lebensmittel noch erhältlich sind. Außerdem würden sich nach dieser Annahme kommunale Netzwerke gründen, wo Hilfe gebündelt und organisiert wird. Es wird angesprochen, dass soziale Netzwerke bereits in Nicht-Krisenzeiten etabliert werden müssten, um ein Bewusstsein für derartige Risiken zu verankern. Das Problem ist, dass Bürger in einem Notfall nicht wissen, an wen sie sich wo wenden können und woher sie welche Informationen bekommen. Solch ein Wissen existiert nach Meinung des Interviewpartners zwar im medizinischen Bereich, aber nicht für einen Lebensmittelengpass $(\mathrm{J} 1)$. 


\subsection{Mediale Logik bei Risiken und Krisen: Ergebnisse der Online- Befragung}

\subsubsection{Berufliches Profil der befragten Journalisten}

Die Stichprobe lässt sich durch Angaben zur Mediengattung, zum Medienressort, zum Beschäftigungsverhältnis, zum Alter der Befragten sowie zur Dauer der Beschäftigung beschreiben. Bei den Kategorien „Mediengattung“ und „Medienressort" war eine Mehrfachauswahl möglich.

\begin{tabular}{|l|r|r|}
\hline Mediengattung & $\mathbf{n} \mathbf{1 1 5}$ & $\mathbf{\%}$ \\
\hline Tageszeitung & 52 & $45,22 \%$ \\
\hline Online-Medium & 31 & $26,96 \%$ \\
\hline Zeitschrift & 16 & $13,91 \%$ \\
\hline Öffentlich-rechtliches Fernsehen & 12 & $10,43 \%$ \\
\hline Öffentlich-rechtlicher Rundfunk & 12 & $10,43 \%$ \\
\hline Sonntags- und Wochenzeitungen & 8 & $6,96 \%$ \\
\hline Nachrichtenagentur & 5 & $4,35 \%$ \\
\hline Privates Fernsehen & 5 & $4,35 \%$ \\
\hline Andere & 3 & $2,61 \%$ \\
\hline Medienressort & $\mathbf{1 1 5}$ & $\mathbf{\%}$ \\
\hline Politik & 46 & $40,00 \%$ \\
\hline Wirtschaft & 28 & $24,35 \%$ \\
\hline Wissenschaft/Forschung & 24 & $20,87 \%$ \\
\hline Lokales & 19 & $16,52 \%$ \\
\hline Kein festes Ressort & 18 & $15,65 \%$ \\
\hline Gesundheit & 13 & $11,30 \%$ \\
\hline Ernährung/Lebensmittel & 9 & $7,83 \%$ \\
\hline Anderes & 10 & $8,70 \%$ \\
\hline Beschäftigungsverhältnis & $\mathbf{n} \%$ \\
\hline Feste Anstellung & 94 & $82,46 \%$ \\
\hline Freie Mitarbeit & 19 & $16,67 \%$ \\
\hline Keine Angabe & 1 & $0,88 \%$ \\
\hline Alter der Befragten & 2 & $\mathbf{\%}$ \\
\hline 20 bis 29 Jahre & 21 & $1,80 \%$ \\
\hline 30 bis 39 Jahre & 44 & $18,92 \%$ \\
\hline 40 bis 49 Jahre & 13 & $26,13 \%$ \\
\hline 50 bis 59 Jahre & $\mathbf{1 1 1}$ & $11,71 \%$ \\
\hline 60 bis 69 Jahre & $\mathbf{n} \%$ \\
\hline
\end{tabular}




\begin{tabular}{|l|r|r|}
\hline 70 bis 79 Jahre & 2 & $1,80 \%$ \\
\hline Dauer der Beschäftigung & $\mathbf{n = 1 1 4}$ & $\mathbf{\%}$ \\
\hline 1 bis 10 Jahre & 17 & $14,91 \%$ \\
\hline 11 bis 20 Jahre & 31 & $27,19 \%$ \\
\hline 21 bis 30 Jahre & 47 & $41,23 \%$ \\
\hline 31 bis 40 Jahre & 16 & $14,04 \%$ \\
\hline 41 bis 50 Jahre & 3 & $2,63 \%$ \\
\hline
\end{tabular}

Tab. 12: Kennzahlen zum beruflichen Profil der befragten Journalisten

Die beiden am stärksten vertretenen Mediengattungen in der befragten Gruppe sind Tageszeitungen $(45,22 \%)$ und Online-Medien (26,96\%). Zwischen 10 bis 15 Prozent der befragten Journalisten sind für das Fernsehen, den öffentlichrechtlichen Rundfunk oder Zeitschriften tätig. Vergleichweise wenige der Befragten sind für eine Sonntags- oder Wochenzeitung oder eine Nachrichtenagentur tätig. Drei Journalisten gaben in einem freien Feld unter „Andere“ an, für ein Fachmedium tätig zu sein (2,61 \%).

Die meisten der befragten Journalisten sind für das Politikressort tätig (40,00 \%), gefolgt von den Ressorts Wirtschaft $(24,35 \%)$ und Wissenschaft/Forschung $(20,87 \%)$. Fast neun Prozent gaben an, in einem anderen Ressort tätig zu sein, darunter: Kultur (3x), Reportage $(2 \mathrm{x})$, Chefredaktion, Investigation, Logistik, Online.

Der größte Teil der Befragten befindet sich in fester Anstellung, etwa 17 Prozent sind frei beruflich journalistisch tätig. Das Durchschnittsalter der Befragten beträgt rund 49 Jahre, die durchschnittliche Dauer der Beschäftigung liegt bei rund 22 Jahren. Diese Angaben korrelieren miteinander $(\mathrm{r}=0,83$; Korrelation nach Pearson, $\mathrm{p}<0,01)$. 


\subsubsection{Ziele der Berichterstattung}

Um Auskunft über das berufliche Selbstverständnis und die damit einhergehenden Ziele, die Journalisten mit ihrer Berichterstattung verfolgen, zu erhalten, sollten die befragten Journalisten angeben, inwiefern die vorgegebenen Aussagen zutreffen oder nicht.

In meinem Beruf als Journalist/in

geht es mir darum, ...

... komplexe Sachverhalte zu erklären.

... das Publikum neutral und präzise zu informieren.

$99,20 \%$

$0,80 \%$

... Kritik an Missständen zu üben.

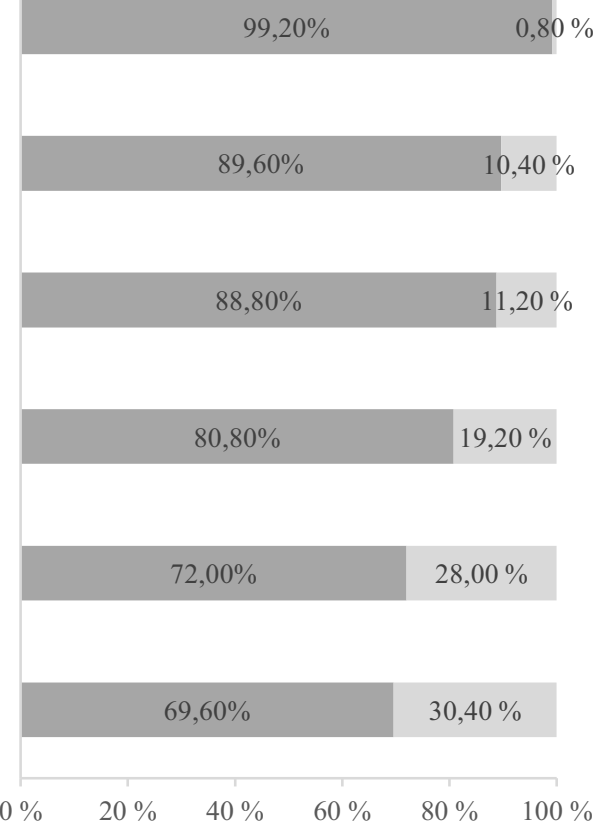

... eine Kontrollinstanz für Politik und Wirtschaft zu sein.

... das Publikum möglichst schnell zu informieren.

... ein Ratgeber für das Publikum zu sein.

... mich auf Nachrichten zu konzentrieren, die für ein möglichst breites Publikum interessant sind.

Zustimmung $\square$ Keine Zustimmung

Abb. 4: Ziele der Berichterstattung, Zustimmung zu Aussagen ( $n=125)$

Frage: Worum geht es Ihnen ganz persönlich in Ihrem Beruf als Journalist/in?;

Wertebereich $1=$,Trifft gar nicht zu“; 2 = „Trifft eher nicht zu“; 3 = ,Trifft eher zu“, 4 = „Trifft voll zu“. Angabe „Zustimmung“ = Kumulierte Werte aus „Trifft voll zu“ und „Trifft eher zu“; Angabe „Keine Zustimmung“ = Kumulierte Werte aus „Trifft gar nicht zu“ und „Trifft eher nicht zu“ 
Das Publikum neutral und präzise zu informieren sowie komplexe Sachverhalte zu erklären, gelten als die wichtigsten Ziele, die die befragten Journalisten mit ihrer Tätigkeit verfolgen (beide nahezu $100 \%$ Zustimmung). Ebenfalls wichtige Ziele sind, Kritik an Missständen zu üben (89,60\%) und als Kontrollinstanz für Politik und Wirtschaft zu fungieren (88,80\%). Es folgen mit noch immer hoher, jedoch vergleichsweise abnehmender Zustimmung: das Publikum möglichst schnell informieren (80,80\%), ein Ratgeber für das Publikum sein $(72,00 \%)$ und sich auf Nachrichten für ein möglichst breites Publikum konzentrieren (69,60\%) (vgl. Abb. 4).

Die Ergebnisse zeigen, dass originäre Funktionen des Journalismus wie die Informationsvermittlung und Aufklärung die wichtigste Rolle für die Ausübung der journalistischen Tätigkeit spielen. Gleichzeitig wird deutlich, dass auch die anderen Ziele von hoher Bedeutung sind. 


\subsubsection{Ziele der Krisenberichterstattung}

Ein zentrales Erkenntnisinteresse betrifft die Ziele, die Journalisten mit ihrer Krisenberichterstattung verfolgen.

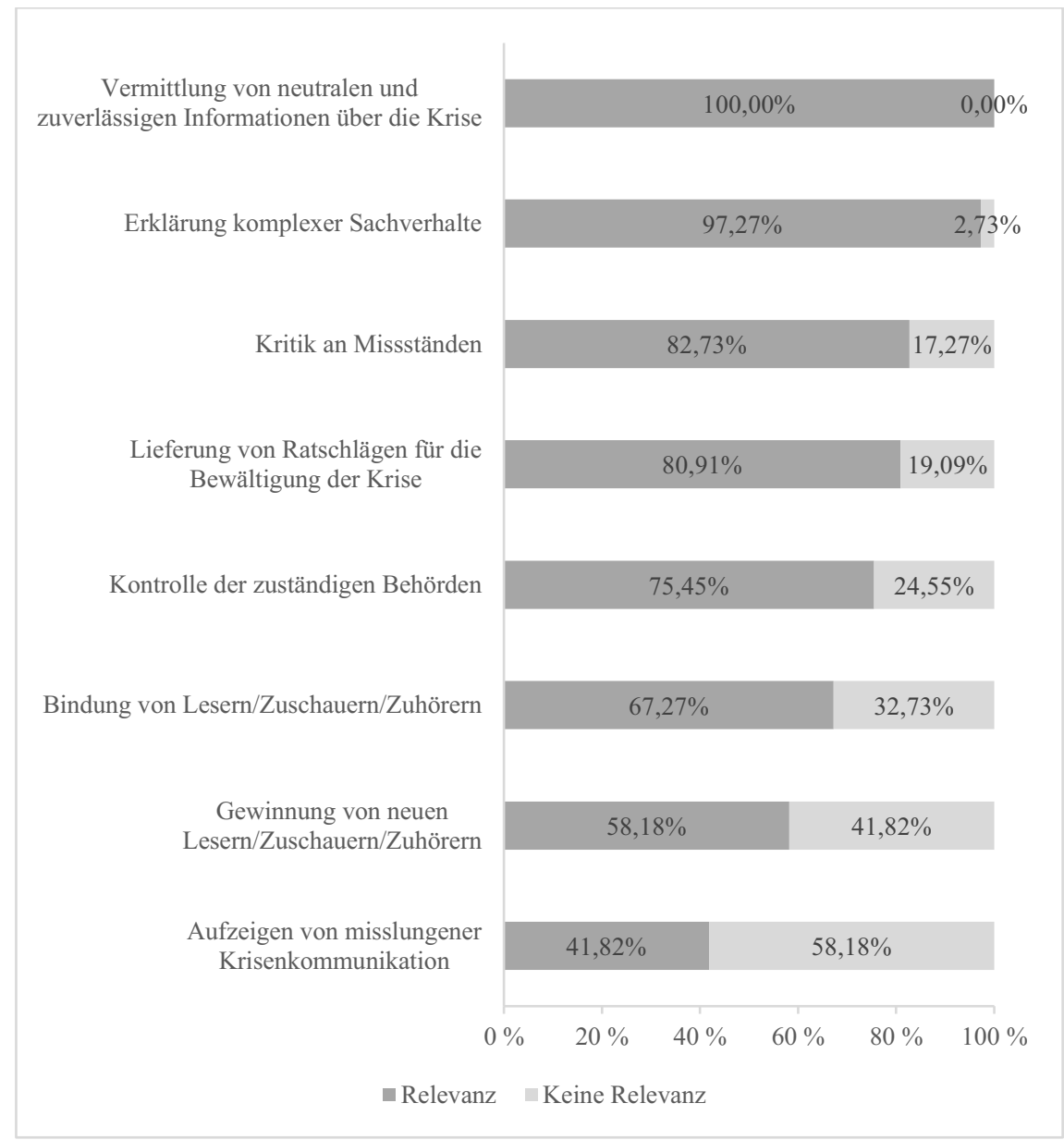

Abb. 5: Ziele der Krisenberichterstattung, Einschätzung der Relevanz $(n=110)$

Frage: Wenn Sie über Krisen berichten, wie wichtig sind Ihnen dabei die nachstehenden Ziele?; Wertebereich 1 = „Überhaupt nicht wichtig“; 2 = „Eher nicht wichtig“; 3 = „Eher wichtig“, 4 = „Sehr wichtig“. Angabe „Relevanz“ = Kumulierte Werte aus „Sehr wichtig“ und „Eher wichtig“; Angabe „Keine Relevanz““ = Kumulierte Werte aus „Überhaupt nicht wichtig“ und „Eher nicht wichtig“ 
Alle befragten Journalisten möchten mit ihrer Krisenberichterstattung neutrale und zuverlässige Informationen über die Krise vermitteln (100\%). Ebenso wichtig ist es ihnen, komplexe Sachverhalte zu erklären $(97,27 \%)$. Kritik an Missständen zu üben, ist für mehr als acht von zehn der befragten Journalisten von Bedeutung $(82,73 \%)$ und noch immer drei Viertel der Befragten verfolgen mit der Krisenberichterstattung das Ziel, zuständige Behörden zu kontrollieren $(75,45 \%)$. Acht von zehn der befragten Journalisten möchten Ratschläge für die Bewältigung der Krise liefern (80,91 \%). Etwa zwei Drittel der Befragten verfolgen bei der Krisenberichterstattung das Ziel, das Publikum an sich zu binden $(67,27 \%)$, mehr als die Hälfte möchte neue Leser, Zuschauer oder Zuhörer gewinnen (58,18 \%). Das Aufzeigen misslungener Krisenkommunikation ist für 41,82 Prozent der Befragten ein wichtiges Ziel der Krisenberichterstattung (vgl. Abb. 5).

\section{Ziele der Krisenberichterstattung nach ökonomischem Interesse}

Tendieren Journalisten, denen es wichtig ist, ein möglichst großes Publikum zu erreichen, in der Krise dazu, dieses ökonomische Interesse verstärkt zu verfolgen?

Diese Frage, die sich aus den theoretischen Ausführungen herleitet, wurde überprüft, indem für die Items, die in der Krise die Ansprache eines möglichst großen Publikums implizieren (Bindung und Vergrößerung des Publikums, Lieferung von Ratschlägen) die Relevanzwerte der Journalisten mit bzw. ohne ein ökonomisches Interesse im Alltag verglichen wurden. Die Lieferung von Ratschlägen kann so verstanden werden, dass damit vor allem Service gegenüber dem Publikum geboten werden soll, so dass sich dieses in der Folge stärker an das Medium gebunden fühlt. Die Gruppen der Journalisten mit bzw. ohne ökonomischem Interesse wurden definiert, indem die Fälle betrachtet wurden, die beim allgemeinen Selbstverständnis dem Item „... mich auf Nachrichten konzentrieren, die für ein möglichst breites Publikum von Interesse sind.“ zugestimmt bzw. nicht zugestimmt haben (ökonomisches Interesse $n=75$; kein ökonomisches Interesse, $n=32$ ) (vgl. Abb. 6). 
Bindung von Lesern/Zuschauern/Zuhörern

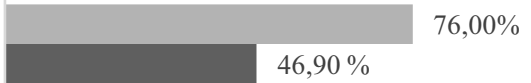

$46,90 \%$

Gewinnung von neuen Lesern/Zuschauern/Zuhörern

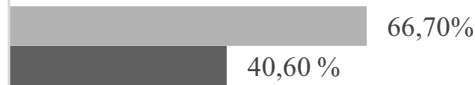

Lieferung von Ratschlägen für die Bewältigung der Krise

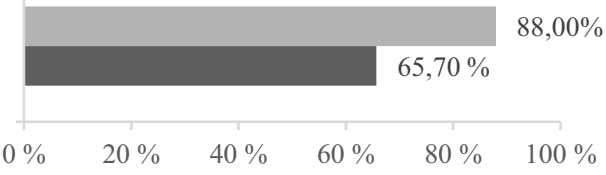

匹ournalisten, die ein ökonomisches Interesse verfolgen $(\mathrm{n}=75)$

- Journalisten, die kein ökonomisches Interesse verfolgen $(\mathrm{n}=32)$

Abb. 6: Ziele der Krisenberichterstattung nach ökonomischem Interesse, Einschätzung der Relevanz

Frage: Wenn Sie über Krisen berichten, wie wichtig sind Ihnen dabei die nachstehenden Ziele?;

Wertebereich 1 = „Überhaupt nicht wichtig“; 2 = ,Eher nicht wichtig“; 3 = „Eher wichtig“, 4 = „Sehr wichtig“. Angabe „Relevanz“ = Kumulierte Werte aus „Sehr wichtig“ und „Eher wichtig“"

Die Ergebnisse zeigen, dass Journalisten, die bereits im Alltag ein ökonomisches Interesse verfolgen, dieses in der Krise intensiviert verfolgen. So hat diese Journalistengruppe die drei Items „Bindung bzw. Gewinnung von Lesern/Zuschauern/Zuhörern“ und „Lieferung von Ratschlägen“ jeweils wichtiger bewertet als die Gruppe ohne ökonomischem Interesse $(76,00 \%$ vs. $46,90 \% ; 66,70 \%$ vs. $40,60 \% ; 88,00 \%$ vs. $65,70 \%)$.

\section{Ziele der Krisenberichterstattung nach Mediengattungen Tageszeitung und Online-Medium}

Für die Ziele der Krisenberichterstattung Bindung und Vergrößerung des Publikums lassen sich Unterschiede hinsichtlich der Mediengattungen feststellen. 


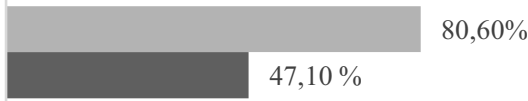

Gewinnung von neuen Lesern/Zuschauern/Zuhörern

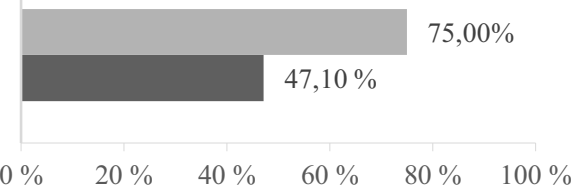

- Tageszeitung $(\mathrm{n}=36) \quad$ Online-Medium $(\mathrm{n}=17)$

Abb. 7: Ziele der Krisenberichterstattung nach Mediengattungen Tageszeitung und Online-Medium, Einschätzung der Relevanz

Frage: Wenn Sie über Krisen berichten, wie wichtig sind Ihnen dabei die nachstehenden Ziele?;

Wertebereich 1 = „Überhaupt nicht wichtig“; 2 = „Eher nicht wichtig“; 3 = „Eher wichtig“, 4 = „Sehr wichtig“. Angabe „Relevanz“ = Kumulierte Werte aus „Sehr wichtig“" und „Eher wichtig“"

Die Ziele Leser, Zuschauer oder Zuhörer in der Krise an sich zu binden oder neue zu gewinnen, werden eher von Journalisten verfolgt, die für Tageszeitungen arbeiten als von Journalisten, die für Online-Medien arbeiten $(80,60 \%$ vs. $47,10 \% ; 75,00 \%$ vs. $47,10 \%)^{37}$. Dieses Ergebnis lässt sich dahingehend interpretieren, dass Tageszeitungen unter einem vergleichsweise höheren ökonomischen Druck stehen und deshalb eher versuchen, Krisen zu nutzen, um das Publikum an sich zu binden respektive zu vergrößern.

\section{Ziele der Krisenberichterstattung nach öffentlich-rechtliche Medien und nicht-öffentlich-rechtliche Medien}

Ein analoger Zusammenhang lässt sich auch in Bezug auf Journalisten öffentlich-rechtlicher Medien und anderer, nicht-öffentlich-rechtlicher Medien identifizieren.

\footnotetext{
${ }^{37}$ Hierfür wurden lediglich die Journalisten berücksichtigt, die ausschließlich für eine Tageszeitung oder ein Online-Medium tätig sind und nicht für beide Gattungen.
} 
Gewinnung von neuen Lesern/Zuschauern/Zuhörern

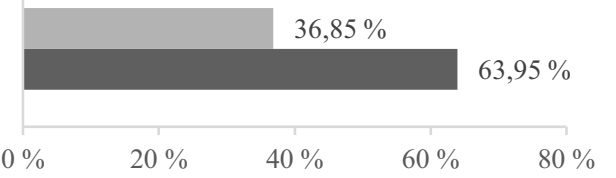

- Journalisten öffentlich-rechtlicher Medien ( $\mathrm{n}=19)$

- Journalisten anderer, nicht-öffentlich-rechtlicher Medien ( $\mathrm{n}=86)$

Abb. 8: Ziele der Krisenberichterstattung nach öffentlich-rechtliche Medien und nicht-öffentlichrechtliche Medien, Einschätzung der Relevanz

Frage: Wenn Sie über Krisen berichten, wie wichtig sind Ihnen dabei die nachstehenden Ziele?;

Wertebereich 1 = „Überhaupt nicht wichtig“; 2 = „Eher nicht wichtig“; 3 = „Eher wichtig“", 4 = „Sehr wichtig“. Angabe „Relevanz“ = Kumulierte Werte aus „Sehr wichtig“ und „Eher wichtig“

Beim Vergleich des Ziels „Bindung von Lesern/Zuschauern/Zuhörern“ schreiben Journalisten von nicht-öffentlich-rechtlichen Medien diesem eine höhere Relevanz zu als Journalisten öffentlich-rechtlicher Medien (70,93\% vs. 57,90\%). Dies zeigt sich bei dem Ziel „Gewinnung von neuen Lesern/Zuschauern/Zuhörern" noch deutlicher: Nahezu zwei Drittel der Journalisten nicht-öffentlichrechtlicher Medien bewerten dieses Ziel als wichtig (63,95\%), hingegen nur gut ein Drittel der Journalisten öffentlich-rechtlicher Medien $(36,85 \%)^{38}$. Dies lässt sich ähnlich interpretieren wie bei dem Ergebnis zuvor: Öffentlich-rechtliche Medien sind nicht dem gleichen ökonomischen Druck ausgesetzt wie andere Medien und konzentrieren sich in der Krise womöglich eher auf ihren gesetzlichen Auftrag der neutralen Informationsvermittlung, wohingegen nichtöffentlich-rechtliche Medien eher versuchen Krisen zu nutzen, um das Publikum an sich zu binden respektive zu vergrößern.

\footnotetext{
${ }^{38}$ Hierfür wurden lediglich die Journalisten berücksichtigt, die ausschließlich für ein öffentlich-rechtliches Medium bzw. ausschließlich für ein nicht-öffentlich-rechtliches Medium tätig sind und nicht für beide Gattungen.
} 


\subsubsection{Ziele der Berichterstattung allgemein und in der Krise}

Aufgrund bisheriger wissenschaftlicher Erkenntnisse (vgl. Kap. 5.7) soll empirisch geprüft werden, inwiefern sich die Ziele der alltäglichen Berichterstattung in der Krise möglicherweise ändern. Die Erkenntnisse aus den Leitfadeninterviews mit Journalisten legen nahe, dass sich die Ziele von Journalisten in der Krise nicht grundsätzlich ändern. Dies wird mit den Erkenntnissen der Online-Befragung überprüft, indem die dargelegten Ziele der allgemeinen Berichterstattung mit denen der Krisenberichterstattung verglichen werden (vgl. Abb. 9). 
Komplexe Sachverhalte erklären

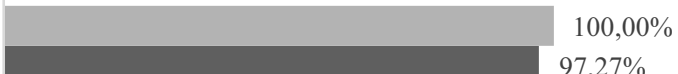

$97,27 \%$

Neutral informieren

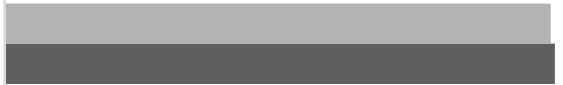

$99,20 \%$

$100,00 \%$

Kritik an Missständen

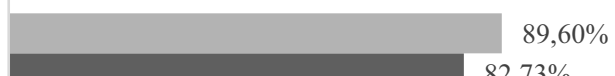

$82,73 \%$

Kontrolle von Politik und Behörden

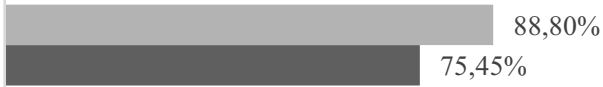

Ratgeber sein

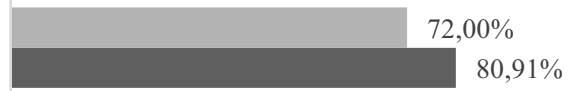

Bindung des Publikums

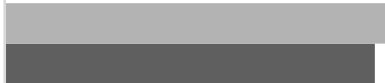

$69,60 \%$

$67,27 \%$

Vergrößerung des Publikums

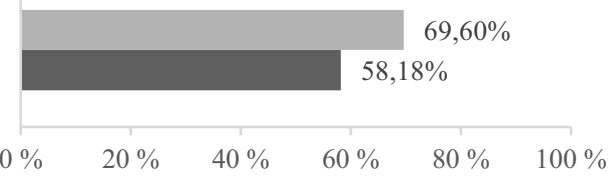

$\begin{array}{llllll}0 \% & 20 \% & 40 \% & 60 \% & 80 \% & 100 \%\end{array}$

$\square$ Ziele der Berichterstattung allgemein $\quad$ ZZiele der Krisenberichterstattung

Abb. 9: Vergleich der Ziele der Berichterstattung allgemein und in der Krise,

Zustimmung zu Aussagen $(n=125) /$ Einschätzung der Relevanz $(n=110)$

Frage Ziele der Berichterstattung allgemein: Worum geht es Ihnen ganz persönlich in Ihrem Beruf als Journalist/in?; Wertebereich $1=$,Trifft gar nicht zu“; 2 = „Trifft eher nicht zu“; 3 = „Trifft eher zu“, $4=$, $T r i f f t$ voll zu“. Angabe „Zustimmung“ = Kumulierte Werte aus „Trifft voll zu“ und „Trifft eher $\mathrm{zu}^{\prime \prime}$

Frage Ziele der Krisenberichterstattung: Wenn Sie über Krisen berichten, wie wichtig sind Ihnen dabei die nachstehenden Ziele?; Wertebereich $1=$,Überhaupt nicht wichtig“; 2 = ,Eher nicht wichtig“; 3 = „Eher wichtig“, 4 = „Sehr wichtig“. Angabe „Relevanz“ = Kumulierte Werte aus „Sehr wichtig" und „Eher wichtig" 
Abbildung 9 zeigt den Vergleich für die kumulierten Zustimmungs- bzw. Relevanzwerte (Werte 3 und 4) der beiden Fragen. Für eine übersichtlichere Darstellung wurden die Items beider Fragen semantisch zusammengefasst.

Insgesamt lässt sich feststellen, dass es keine sehr großen Differenzen zwischen den journalistischen Zielen im Alltag und denen in der Krise gibt. Es zeichnen sich leichte Unterschiede ab, darunter, eine stärkere Ausprägung des Ziels der Ratgebertätigkeit in der Krise im Vergleich zur Serviceorientierung in der allgemeinen Berichterstattung (80,91 \% vs. 72,00 \%). Die journalistischen Kritik- und Kontrollaufgaben sind hingegen im Alltag etwas wichtiger als in der Krise ( $89,62 \%$ vs. $82,73 \%$ bzw. $88,80 \%$ vs. $75,45 \%)$. Auch die Vergrößerung des Publikums stellt mit einer Differenz von über elf Prozent ein relevanteres Ziel im Alltag als in der Krise dar (69,90\% vs. 58,18 \%).

\subsubsection{Berichterstattung über Krisenkommunikation von Behörden}

Inwiefern die Krisenkommunikation der Behörden selbst zum Gegenstand der Berichterstattung würde, war Gegenstand einer Frage, die sich auf das der Arbeit zugrunde liegende Szenario bezog.

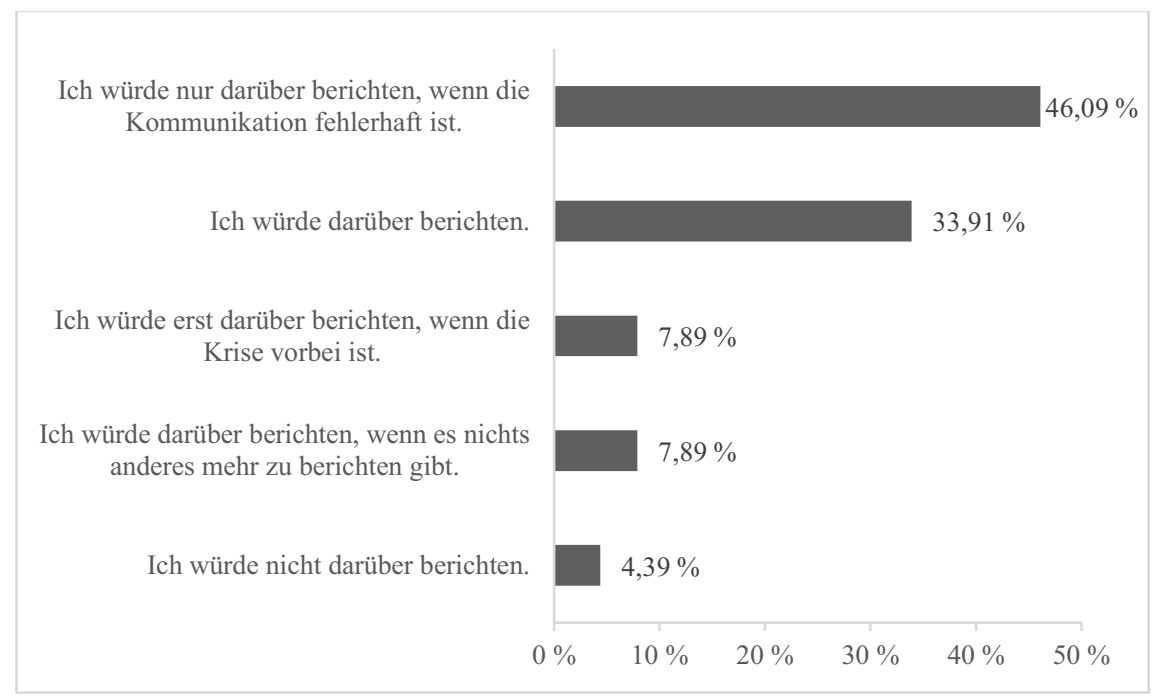

Abb. 10: Berichterstattung über die Krisenkommunikation von Behörden,

Einfachauswahl $(n=115)$

Frage: Inwiefern würden Sie in dieser Situation über die Krisenkommunikation der Behörden berichten? (Szenariofrage) 
Fast jeder zweite befragte Journalist würde die Krisenkommunikation der Behörden in der Berichterstattung thematisieren, wenn diese fehlerhaft ist (46,09\%). Ein Drittel der Befragten gibt an, dass sie in einer Krise grundsätzlich über die Krisenkommunikation der Behörden berichten würden (33,91\%). Etwa acht Prozent der Befragten würden über die Kommunikation berichten, wenn die Krise vorbei ist $(7,89 \%)$ oder wenn es nichts anderes mehr über die Krise zu berichten gibt $(7,89 \%)$. Nur wenige Befragte würden überhaupt nicht über die Krisenkommunikation der zuständigen Behörde berichten (4,39 \%). Es kann geschlussfolgert werden, dass die behördliche Krisenkommunikation ein zentraler Bestandteil der (Post-)Krisenberichterstattung ist.

\subsubsection{Verhältnis Journalisten und Behörden in Krisen}

Die Journalisten wurden gebeten, ihre Zustimmung zu einzelnen Aussagen über ihr Verhältnis zu Behörden und ihren Mitarbeitern in Krisen anzugeben. Die Aussagen leiten sich maßgeblich aus den Erkenntnissen der Leitfadeninterviews ab (vgl. Abb. 11). 
In einer Krise konfrontiere ich die zuständigen Behörden mit kritischen Meinungen von Experten.

Wenn Behörden in eine Krise involviert sind, bin ich ihren Aussagen gegenüber skeptisch.

Wenn die zuständigen Behörden in einer Krise keine Auskunft geben, wende ich mich an andere Personen, auch wenn diese weniger Fachwissen haben.

Das Vertrauensverhältnis zwischen mir und den Mitarbeitern von Behörden ist für meine Arbeit in der Krise sehr wichtig.

In einer extremen Krisen- oder Notsituation wäre ich bereit, mit den zuständigen Behörden zusammenzuarbeiten.

Es wäre gut, wenn es in der Krise eine zentrale behördliche Einrichtung gäbe, die ausschließlich für die externe

Krisenkommunikation zuständig wäre.

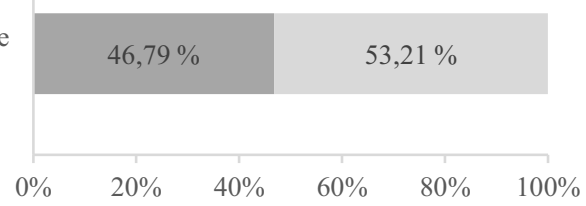

Zustimmung $\quad$ Keine Zustimmung

$80,73 \%$ $19,27 \%$
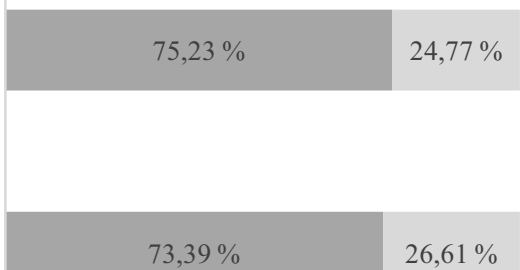

$26,61 \%$

Abb. 11: Verhältnis zwischen Journalisten und Behörden in Krisen, Zustimmung zu Aussagen $(n=109)$

Frage: Wie beurteilen Sie Ihr Verhältnis zu Behörden in Krisensituationen, wenn diese in die Krise involviert sind?; Wertebereich $1=$,Stimme gar nicht zu“; 2 = „Stimme eher nicht zu“; 3 = „Stimme eher zu“, 4 = „Stimme voll zu“. Angabe „Zustimmung“ = Kumulierte Werte aus „Stimme voll zu“ und „Stimme eher zu“; Angabe „Keine Zustimmung“ = Kumulierte Werte aus „Stimme gar nicht zu“ und „Stimme eher nicht zu“ 
Nahezu 90 Prozent der befragten Journalisten stimmen zu, dass sie in einer Krise die zuständigen Behörden mit kritischen Meinungen von Experten konfrontieren würden (88,99 \%). Die hohe Zustimmung ist kongruent zu einer Erkenntnis aus der gleichen Befragung, nach der Journalisten in der Krise versuchen möglichst viele Aussagen von Experten zu erhalten (Drews \& Raupp 2016).

Wenn Behörden in eine Krise involviert sind, wären etwa acht von zehn der befragten Journalisten den Aussagen dieser Behörde gegenüber skeptisch $(81,65 \%)$. Fast genauso hoch ist die Zustimmung zu der Aussage, dass man sich als Journalist, wenn Behörden in der Krise keine Auskunft geben, an andere Personen wendet, auch wenn diese weniger Fachwissen haben $(80,73 \%)$.

Etwa drei Viertel der Befragten stimmen zu, dass das Vertrauensverhältnis zwischen ihnen und Behördenmitarbeitern für ihre Arbeit in Krisen wichtig ist $(75,23 \%)$ sowie dass sie in einer außergewöhnlichen Krisensituation dazu bereit sind, mit den zuständigen Behörden zusammenzuarbeiten (73,39 \%).

Annähernd die Hälfte der befragten Journalisten hält eine zentrale Behörde, die ausschließlich für die externe Krisenkommunikation zuständig ist, für notwendig $(46,79 \%)$.

\section{Bereitschaft zur Zusammenarbeit nach öffentlich-rechtlichen Medien und nicht-öffentlich-rechtlichen Medien}

Die These, dass Journalisten der öffentlich-rechtlichen Medien in einer Krise eher zu einer Zusammenarbeit mit Behörden bereit sind als die Journalisten der anderen Mediengattungen, die sich aus den Erkenntnissen der Leitfadeninterviews herleitet, muss widerlegt werden: Journalisten der öffentlich-rechtlichen Medien stimmen der Aussage zur Zusammenarbeit in der Krise weniger zu $(66,66 \%)$ als Journalisten anderer Medien $(77,38 \%)^{39}$ (vgl. Abb. 12).

\footnotetext{
${ }^{39}$ Hierfür wurden lediglich die Journalisten berücksichtigt, die ausschließlich für ein öffentlichrechtliches Medium bzw. ausschließlich für ein nicht-öffentlich-rechtliches Medium tätig sind und nicht für beide Gattungen.
} 
In einer extremen Krisen- oder Notsituation wäre ich bereit, mit den zuständigen Behörden zusammenzuarbeiten.
$66,66 \%$

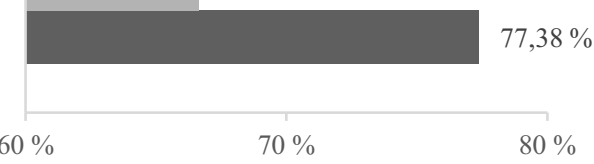

ש Journalisten öffentlich-rechtlicher Medien ( $\mathrm{n}=18)$

- Journalisten anderer, nicht-öffentlich-rechtlicher Medien $(\mathrm{n}=84)$

Abb. 12: Bereitschaft zur Zusammenarbeit nach öffentlich-rechtlichen Medien und nicht-öffentlichrechtlichen Medien, Zustimmung zu Aussage

Frage: Wie beurteilen Sie Ihr Verhältnis zu Behörden in Krisensituationen, wenn diese in die Krise involviert sind?; Wertebereich $1=$ „Stimme gar nicht zu“; $2=$ „Stimme eher nicht zu“; $3=$ „Stimme eher zu“, 4 = „Stimme voll zu“. Angabe „Zustimmung“ = Kumulierte Werte aus „Stimme voll zu“ und ,Stimme eher zu“"

Über die Gründe für diese Unterschiede kann nur spekuliert werden. So erhoffen sich Journalisten nicht-öffentlich-rechtlicher Medien von einer Zusammenarbeit möglicherweise einen besseren Zugang zu exklusiven Informationen. Journalisten öffentlich-rechtlicher Medien ist hingegen eventuell eher daran gelegen, ihrer gesetzlich vorgeschriebenen Neutralität nachzukommen und damit auch zu verdeutlichen, dass sie kein „Staatssender" sind (vgl. Kap. 8.3.5).

\section{Zusammenhänge zwischen den einzelnen Aussagen zum Verhältnis}

Die Aussagen zu dem Verhältnis zu den Behörden und ihren Mitarbeitern spiegeln unterschiedliche Ansichten der Journalisten wider. Dabei sind einige Zusammenhänge zwischen diesen Ansichten erkennbar (vgl. Tab. 13). 


\begin{tabular}{|l|l|l|l|l|l|}
\hline & $\begin{array}{l}\text { Vertrauens- } \\
\text { verhältnis }\end{array}$ & Skepsis & $\begin{array}{l}\text { Kritische } \\
\text { Meinungen }\end{array}$ & $\begin{array}{l}\text { Andere } \\
\text { Personen }\end{array}$ & $\begin{array}{l}\text { Zusammen- } \\
\text { arbeit }\end{array}$ \\
\hline $\begin{array}{l}\text { Vertrauens- } \\
\text { verhältnis }\end{array}$ & - & $-0,10$ & $-0,03$ & $-0,05$ & 0,14 \\
\hline Skepsis & $-0,10$ & - & $0,38^{* *}$ & $0,32 * *$ & $-0,23 * *$ \\
\hline $\begin{array}{l}\text { Kritische } \\
\text { Meinungen }\end{array}$ & $-0,03$ & $0,38^{* *}$ & - & $0,38^{* *}$ & $-0,13$ \\
\hline $\begin{array}{l}\text { Andere } \\
\text { Personen }\end{array}$ & $-0,05$ & $0,32^{* *}$ & $0,38^{* *}$ & - & $-0,10$ \\
\hline $\begin{array}{l}\text { Zusammen- } \\
\text { arbeit }\end{array}$ & 0,14 & $-0,23^{*}$ & $-0,13$ & $-0,10$ & - \\
\hline
\end{tabular}

Tab. 13: Zusammenhänge zwischen den einzelnen Aussagen zum Verhältnis, Korrelationskoeffizient Spearman-Rho $(n=109)$

** Korrelation ist bei Niveau 0,01 signifikant (zweiseitig).

* Korrelation ist bei Niveau 0,05 signifikant (zweiseitig).

Tabelle 13 zeigt die Aussagen zueinander anhand des Korrelationskoeffizienten Spearman-Rho. Folgende Zusammenhänge sind erkennbar:

- $\quad$ Die befragten Journalisten, die den Aussagen von Behörden gegenüber skeptisch sind, konfrontieren die Behörden in der Krise auch eher mit kritischen Meinungen von Experten $\left(\mathrm{r}_{\mathrm{s}}=0,38\right)$.

- Die befragten Journalisten, die den Aussagen von Behörden gegenüber skeptisch sind, wenden sich in der Krise eher an andere Personen, wenn die Behörden in der Krise keine Auskunft geben $\left(r_{s}=0,32\right)$.

- Umgekehrt ist zu erkennen, dass die befragten Journalisten, die den Aussagen von Behörden gegenüber skeptisch sind, in der Krise weniger $\mathrm{zu}$ einer Zusammenarbeit bereit sind $\left(\mathrm{r}_{\mathrm{s}}=-0,23\right)$.

- Die befragten Journalisten, die Behörden mit kritischen Meinungen von Experten konfrontieren, wenden sich in der Krise eher an andere Personen, wenn die Behörden in der Krise keine Auskunft geben $\left(r_{s}=0,38\right)$.

Es liegen keine sehr starken Zusammenhänge zwischen den Aussagen zum Verhältnis vor, Werte zwischen 0,2 und 0,4 deuten auf nur schwache Korrelationen hin. Dennoch lassen diese bereits den vorsichtigen Rückschluss zu, dass von den befragten Journalisten solche darunter sind, die gegenüber Behörden in Krisen grundlegend skeptisch sind und die Behörden entsprechend stärker unter Druck setzen. Im Gegensatz dazu gibt es Journalisten, die das Verhältnis zu Behörden weniger kritisch sehen und eher zu einer Zusammenarbeit bereit sind. 


\subsubsection{Erwartungen an Krisenkommunikation von Behörden}

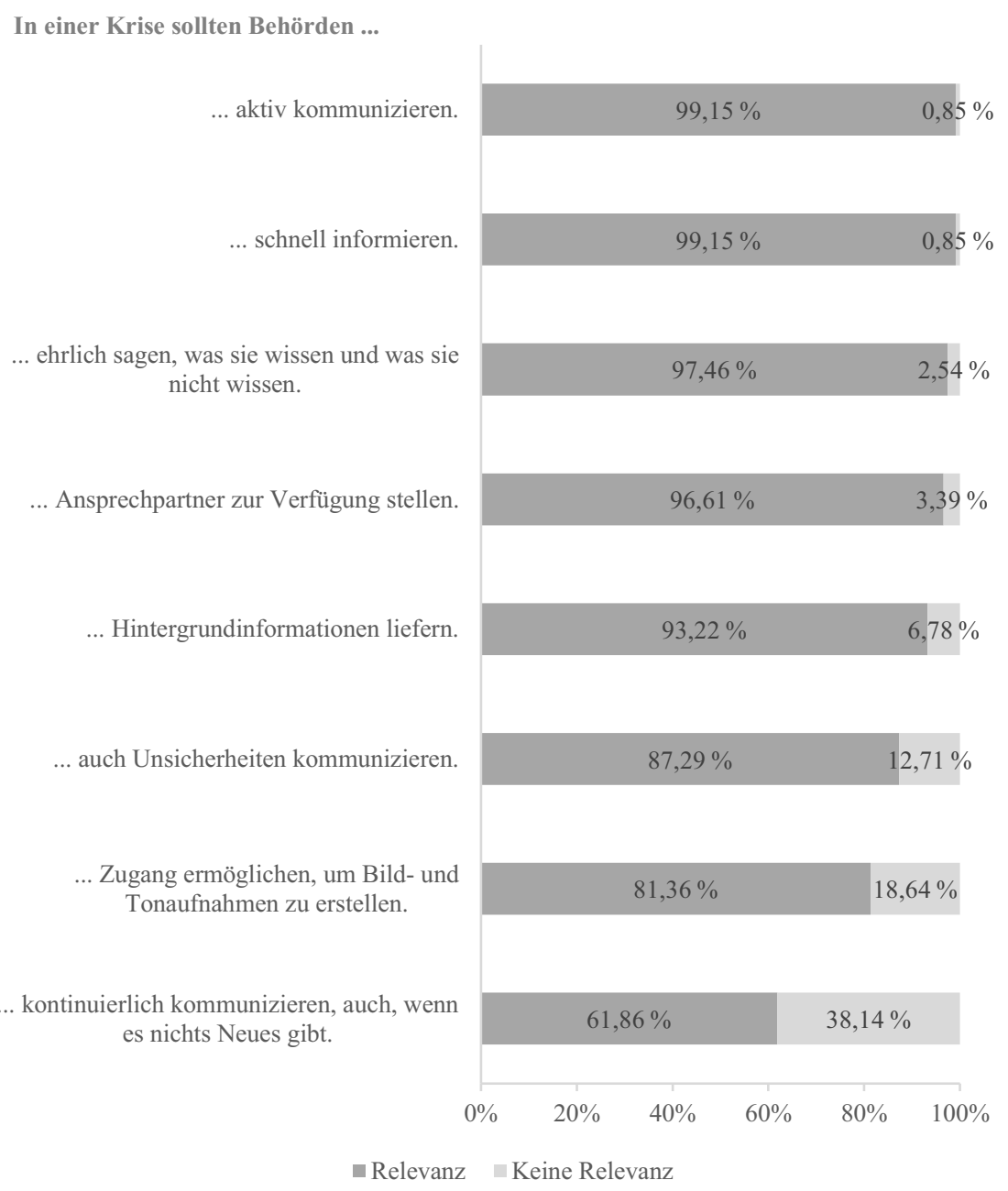

Abb. 13: Erwartungen an Krisenkommunikation von Behörden, Einschätzung der Relevanz $(n=118)$

Frage: Was erwarten Sie in einer Krise von der Kommunikation der zuständigen Behörden gegenüber Medien?; Wertebereich 1 = „Überhaupt nicht wichtig“; 2 = „Eher nicht wichtig“; 3 = „Eher wichtig“, $4=$,Sehr wichtig“. Angabe „Relevanz“ = Kumulierte Werte aus ,Sehr wichtig“" und „Eher wichtig“; Angabe „Keine Relevanz“ = Kumulierte Werte aus „Überhaupt nicht wichtig“ und „Eher nicht wichtig“" 
Die Ermittlung der journalistischen Erwartungen an die behördliche Krisenkommunikation stellt ein zentrales Erkenntnisinteresse der Arbeit dar. Es wurde zunächst gefragt, wie wichtig den Journalisten bestimmte Erwartungen, die sich vor allem aus den Leitfadeninterviews ableiten, sind (vgl. Abb. 13).

Nahezu allen Befragten ist es wichtig, dass Behörden in einer Krise gegenüber den Medien aktiv kommunizieren (99,15\%), diese schnell informieren $(99,15 \%)$ sowie dass Behörden ehrlich sagen, was sie wissen und was nicht $(97,46 \%)$. Weiterhin halten es über 90 Prozent für wichtig, dass Behörden den Medien in Krisen einen Ansprechpartner zur Verfügung stellen (96,16\%) und Hintergrundinformationen liefern (93,22\%). Rund 87 Prozent der Befragten finden es eher oder sehr wichtig, dass auch Unsicherheiten kommuniziert werden. Noch immer über 80 Prozent erwarten von Behörden, dass sie Zugang zu Bild- und Tonaufnahmen ermöglichen $(81,36 \%)$ und etwa sechs von zehn Befragten geben an, Behörden sollten kontinuierlich kommunizieren, auch dann, wenn es nichts Neues gibt $(61,86 \%)$.

Zudem hatten die Journalisten die Möglichkeit, in einem offenen Feld „Sonstiges" weitere Erwartungen anzugeben. Ein Befragter weist darauf hin, dass es wichtig ist, Kommunikationsverantwortliche mit Medienerfahrung als Ansprechpartner zur Verfügung zu stellen.

\section{Besonders wichtige Aspekte behördlicher Krisenkommunikation}

Im Anschluss sollten die befragten Journalisten die genannten Erwartungen noch einmal betrachten und angeben, welche Aspekte der behördlichen Krisenkommunikation für sie besonders wichtig sind. Dabei konnten sie von den zuvor bereits abgefragten Items maximal drei auswählen (ohne Skalenniveaus). Diese Frage wurde gestellt, da der Pretest bereits hohe Relevanzwerte für die Erwartungen offenbarte und auf diese Art und Weise eine eindeutigere Differenzierung erzielt werden sollte (vgl. Abb. 14). 
Ehrliche, transparente Kommunikation

Schnelle Informationen

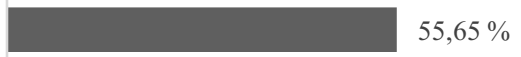

Aktive Kommunikation

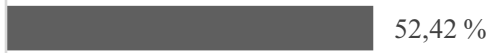

Ansprechpartner zur Verfügung stellen

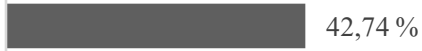

Lieferung von Hintergrundinformationen

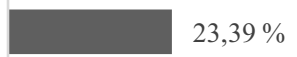

Kommunikation von Unsicherheiten

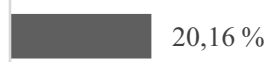

Kontinuierliche Kommunikation

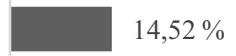

Zugang zu Bild- und Tonaufnahmen

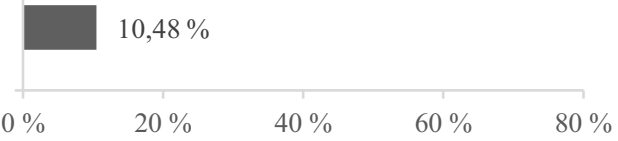

Abb. 14: Besonders wichtige Aspekte behördlicher Krisenkommunikation, Mehrfachauswahl möglich (max. drei) $(n=124)$

Frage: Wenn Sie die Erwartungen noch einmal betrachten: Welche der genannten Aspekte der Krisenkommunikation von Behörden sind aus Ihrer Sicht besonders wichtig?

Durch diese Ergebnisse lassen sich die folgenden drei journalistischen Erwartungen als die relevantesten festhalten: eine ehrliche und transparente $(74,19 \%)$, eine schnelle $(55,65 \%)$ und eine aktive $(52,42 \%)$ behördliche Krisenkommunikation. 


\subsubsection{Erwartungen an zwischenbehördliche Krisenkommunikation}

Die Journalisten wurden außerdem nach ihren Erwartungen an die zwischenbehördliche Krisenkommunikation gefragt.

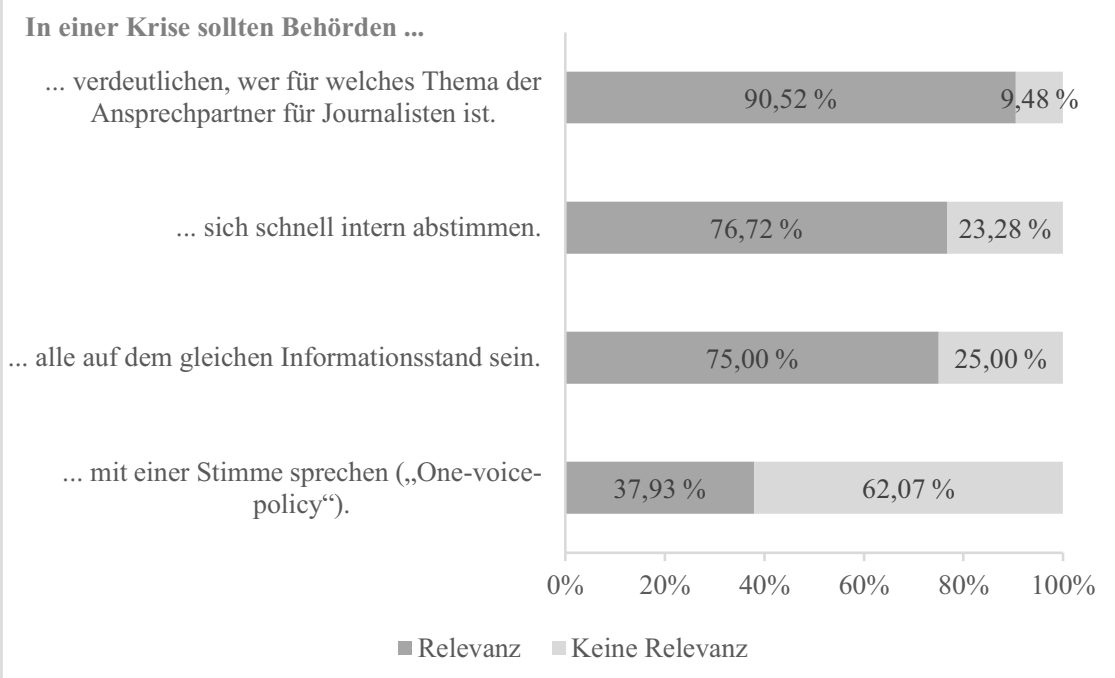

Abb. 15: Erwartungen an zwischenbehördliche Krisenkommunikation, Einschätzung der Relevanz $(n=116)$

Frage: Was erwarten Sie, wenn in einer Krise mehrere Behörden (auch auf Bundes- und Landesebene) für die Kommunikation verantwortlich sind?; Wertebereich $1=$ „Überhaupt nicht wichtig“; 2 = „Eher nicht wichtig“; 3 = „Eher wichtig“, 4 = „Sehr wichtig“. Angabe „Relevanz“ = Kumulierte Werte aus „Sehr wichtig“ und „Eher wichtig“; Angabe „Keine Relevanz“ = Kumulierte Werte aus ,Überhaupt nicht wichtig“ und „Eher nicht wichtig“

Hinsichtlich der Erwartungen an die interorganisationale Krisenkommunikation von Behörden ist es einem Großteil der befragten Journalisten wichtig, dass Behörden verdeutlichen, wer für welches Thema der richtige Ansprechpartner für Journalisten ist $(90,52 \%)$. Etwa drei Viertel finden es wichtig, dass sich Behörden schnell intern abstimmen (76,72 \%) sowie dass alle Behörden auf dem gleichen Informationsstand sind $(75,00 \%)$. Nur etwas mehr als ein Drittel $(37,93 \%)$ findet es wichtig, dass Behörden in der Krise mit einer Stimme sprechen und damit die One-voice-policy befolgen (vgl. Abb. 15). 


\subsubsection{Erwartungen an Risikokommunikation von Behörden}

\section{Zusätzlich wurden die journalistischen Erwartungen an die behördliche Risiko- kommunikation ermittelt.}

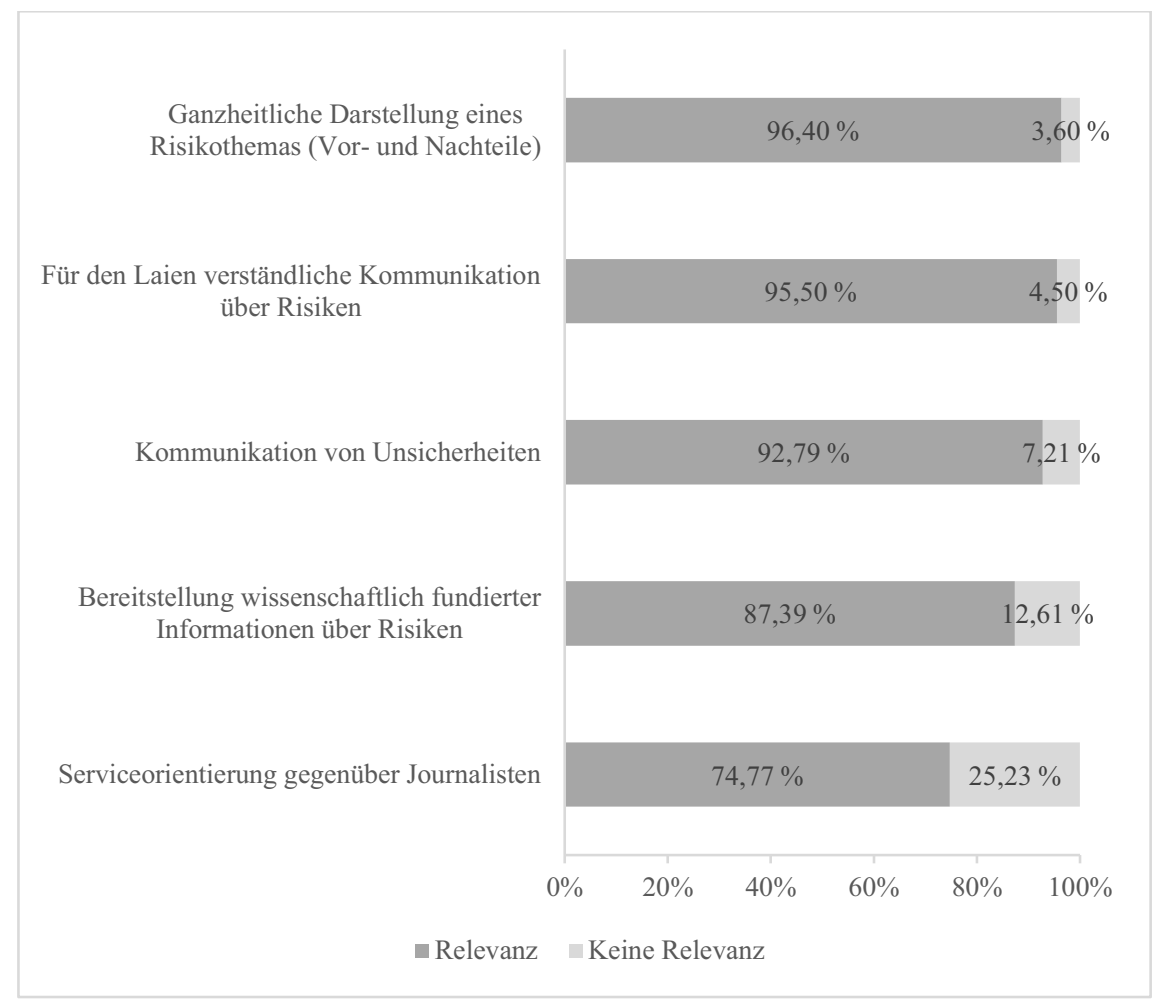

Abb. 16: Erwartungen an Risikokommunikation von Behörden, Einschätzung der Relevanz $(n=111)$

Frage: Was ist Ihnen wichtig, wenn Behörden über Risiken kommunizieren (z. B. Risiko von Krankheitserregern in Lebensmitteln, Risiko einer Grippepandemie, Risiken neuer Technologien)?; Wertebereich 1 = „Überhaupt nicht wichtig“; 2 = „Eher nicht wichtig“; $3=$ „Eher wichtig“, 4 = „Sehr wichtig“. Angabe „Relevanz“ = Kumulierte Werte aus „Sehr wichtig“" und „Eher wichtig“; Angabe „Keine Relevanz“ = Kumulierte Werte aus „Überhaupt nicht wichtig“ und „Eher nicht wichtig“ 
Nahezu alle befragten Journalisten halten es für wichtig, dass Risikothemen ganzheitlich dargestellt werden (96,40\%). Ebenso wichtig ist den Journalisten eine Kommunikation über Risiken, die auch für Laien verständlich ist $(95,50 \%)$. Rund neun von zehn der befragten Journalisten geben außerdem an, dass ihnen eine Kommunikation über Unsicherheiten (92,79\%) sowie die Bereitstellung wissenschaftlich fundierter Informationen über Risiken $(87,39 \%)$ wichtig ist. Dass Behörden gegenüber Journalisten serviceorientiert agieren, bewerten die Journalisten im Vergleich $\mathrm{zu}$ den anderen abgefragten Aspekten zwar als weniger wichtig, dennoch ist noch immer drei Viertel der befragten Journalisten Serviceorientierung wichtig (74,77 \%) (vgl. Abb. 16).

\subsubsection{Eintrittswahrscheinlichkeit eines Lebensmittelengpasses}

Aufgrund eines geringen Risikobewusstseins für einen Lebensmittelengpass, das durch die Leitfadeninterviews zum Ausdruck gebracht wurde, wurde nach der Eintrittswahrscheinlichkeit eines solchen gefragt.

\begin{tabular}{|r|r|r|}
\hline $\begin{array}{l}\text { Einschätzung des Eintritts eines Lebensmittelengpasses } \\
\text { als... }\end{array}$ & $\mathbf{n}$ & $\mathbf{\%}$ \\
\hline$\ldots$ sehr unwahrscheinlich (Wert 1) & 45 & $39,47 \%$ \\
\hline (Wert 2) & 41 & $35,97 \%$ \\
\hline (Wert 3) & 24 & $21,05 \%$ \\
\hline (Wert 4) & 4 & $3,51 \%$ \\
\hline$\ldots$ sehr wahrscheinlich (Wert 5) & 0 & $0,00 \%$ \\
\hline
\end{tabular}

Tab. 14: Einschätzung der Eintrittswahrscheinlichkeit eines Lebensmittelengpasses $(n=114)$

Frage: Für wie wahrscheinlich halten Sie es, dass es in Deutschland zu einer Situation kommt, in der die Versorgung mit Lebensmitteln nicht mehr gewährleistet ist?

Die befragten Journalisten halten es im Großen und Ganzen für unwahrscheinlich, dass es in Deutschland zu einer Situation kommt, in der die Versorgung mit Lebensmitteln nicht mehr gewährleistet ist (M: 1,89; SD: 0,86). Nur 3,51 Prozent halten dies für wahrscheinlich, während drei Viertel der Befragten das Eintreten einer solchen Krise als unwahrscheinlich einschätzen (75,44\%).

Mit diesem empirischen Beleg muss nicht nur von einem geringen, sondern von einem nicht vorhandenen Risikobewusstsein gegenüber einem Lebensmittelengpass ausgegangen werden. 